\title{
Enantioselective Construction of Carbobicyclic Scaffolds
}

Douglass F. Taber, Yigang He, Ming Xu

Department of Chemistry and Biochemistry, University of Delaware, Newark, DE 19716

\section{Supporting Information}

\section{Contents:}

S-1 to S-5 Table of Contents

S-6 General Experimental

S-7 Epoxide 1a

S-8 Alcohol 2a, alcohol 2b

S-9 Alcohol 2c, alcohol 2d

S-10 Alcohol 2e

S-11 Alcohol 2f, benzoate $\mathbf{6 b}$

S-12 (t) - Alcohol 2a

S-13 (+) - Diol 20

S-14 (-) - Diol 21

S-15 (-) - Benzyl ether 22

S-16 (-) - Aldehyde 23

S-17 (-) - Benzyl ether 24

S-18 (+) - Diol 25, (-) - aldehyde 26

S-19 (-) - Ketone 27

S-20 (-)- Ketone 8, tosylate 28

S-21 Aldehyde 9 
S-22 Aldehyde 10

S-23 Benzenesulfonate 29

S-24 Methanesulfonate 13

S-25 Sulfone 11

S-26 Sulfone 12

S-27 Sulfone 3

S-28 Epoxide 30

S-29 Ketone 31

S-30 Ketal 14

S-31 Sulfone 15

S-32 Benzenesulfonate 32, methanesulfonate 16

S-33 Sulfone 17, benzenesulfonate 33

S-34 Methanesulfonate 18

S-35 Sulfone 19, references

S-36 $\quad{ }^{1} \mathrm{H}$ NMR of 1a

S-37 $\quad{ }^{13} \mathrm{C}$ NMR of 1a

S-38 $\quad{ }^{1} \mathrm{H}$ NMR of $2 \mathrm{a}$

S-39 $\quad{ }^{13} \mathrm{C}$ NMR of $2 a$

S-40 HPLC of $(+)$ - 2a

S-41 $\quad{ }^{1} \mathrm{H}$ NMR of $\mathbf{2 b}$

S-42 $\quad{ }^{13} \mathrm{C}$ NMR of $\mathbf{2 b}$

S-43 $\quad{ }^{1} \mathrm{H}$ NMR of $2 \mathrm{c}$

S-44 $\quad{ }^{13} \mathrm{C}$ NMR of $2 \mathrm{c}$ 
S-46 $\quad{ }^{13}$ C NMR of $2 d$

S-47 $\quad{ }^{1} \mathrm{H}$ NMR of $2 f$

S-48 $\quad{ }^{13} \mathrm{C}$ NMR of $2 f$

S-49 $\quad{ }^{1} H$ NMR of $6 b$

S-50 $\quad{ }^{13} \mathrm{C} \mathrm{NMR}$ of $6 \mathrm{~b}$

S-51 $\quad{ }^{1}$ H NMR of 20

S-52 ${ }^{13} \mathrm{C}$ NMR of 20

S-53 $\quad{ }^{1} \mathrm{H}$ NMR of 21

S-54 $\quad{ }^{13}$ C NMR of 21

S-55 $\quad{ }^{1}$ H NMR of 22

S-56 $\quad{ }^{13} \mathrm{C}$ NMR of 22

S-57 $\quad{ }^{1} \mathrm{H}$ NMR of 23

S-58 $\quad{ }^{13} \mathrm{C}$ NMR of 23

S-59 $\quad{ }^{1} H$ NMR of 24

S-60 $\quad{ }^{13}$ C NMR of 24

S-61 ${ }^{1} \mathrm{H}$ NMR of 25

S-62 $\quad{ }^{13} \mathrm{C}$ NMR of 25

S-63 $\quad{ }^{1}$ H NMR of 26

S-64 $\quad{ }^{13}$ C NMR of 26

S-65 $\quad{ }^{1}$ H NMR of 27

S-66 $\quad{ }^{13}$ C NMR of 27

S-67 $\quad{ }^{1} \mathrm{H}$ NMR of 8 
${ }^{1} \mathrm{H}$ NMR of 9

S-72

${ }^{13} \mathrm{C}$ NMR of 9

S-73

${ }^{1} \mathrm{H}$ NMR of 10

S-74

${ }^{13} \mathrm{C} \mathrm{NMR}$ of 10

S-75

${ }^{1} \mathrm{H}$ NMR of 29

S-76

${ }^{13} \mathrm{C}$ NMR of 29

S-77

${ }^{1} \mathrm{H}$ NMR of 13

S-78

${ }^{13} \mathrm{C}$ NMR of 13

S-79

'H NMR of 11

S-80

${ }^{13} \mathrm{C}$ NMR of 11

S-81

${ }^{1} \mathrm{H}$ NMR of 12

S-82

${ }^{13} \mathrm{C}$ NMR of 12

S-83

${ }^{1} \mathrm{H}$ NMR of 3

S-84

${ }^{13} \mathrm{C}$ NMR of 3

S-85

${ }^{1} \mathrm{H}$ NMR of 30

S-86

${ }^{13} \mathrm{C}$ NMR of 30

S-87

${ }^{1} \mathrm{H}$ NMR of 31

S-88

${ }^{13} \mathrm{C} \mathrm{NMR}$ of 31

S-89

${ }^{1} \mathrm{H}$ NMR of 14

S-90

${ }^{13} \mathrm{C} \mathrm{NMR}$ of 14 


$\begin{array}{ll}\text { S-91 } & { }^{1} \mathrm{H} \text { NMR of } \mathbf{1 5} \\ \text { S-92 } & { }^{13} \mathrm{C} \text { NMR of } \mathbf{1 5} \\ \text { S-93 } & { }^{1} \mathrm{H} \text { NMR of } \mathbf{3 2} \\ \text { S-94 } & { }^{13} \mathrm{C} \text { NMR of } \mathbf{3 2} \\ \text { S-95 } & { }^{1} \mathrm{H} \text { NMR of } \mathbf{1 6} \\ \text { S-96 } & { }^{13} \mathrm{C} \text { NMR of } \mathbf{1 6} \\ \text { S-97 } & { }^{1} \mathrm{H} \text { NMR of } \mathbf{1 7} \\ \text { S-98 } & { }^{13} \mathrm{C} \text { NMR of } \mathbf{1 7} \\ \text { S-99 } & { }^{1} \mathrm{H} \text { NMR of } \mathbf{3 3} \\ \text { S-100 } & { }^{13} \mathrm{C} \text { NMR of } \mathbf{3 3} \\ \text { S-101 } & { }^{1} \mathrm{H} \text { NMR of } \mathbf{1 8} \\ \text { S-102 } & { }^{13} \mathrm{C} \text { NMR of } \mathbf{1 8} \\ \text { S-103 } & { }^{1} \mathrm{H} \text { NMR of } \mathbf{1 9} \\ \text { S-104 } & { }^{13} \mathrm{C} \text { NMR of } \mathbf{1 9}\end{array}$




\section{Experimental Section}

General. ${ }^{1} \mathrm{H}$ and ${ }^{13} \mathrm{C}$ NMR were recorded as solutions in $\mathrm{CDCl}_{3}$ at 400 and 100 (or 90) $\mathrm{MHz}$, respectively. ${ }^{13} \mathrm{C}$ NMR multiplicities were determined with the aid of a JVERT sequence, differentiating the signals for methylene and quaternary carbons as "u" from methyl and methine carbons as "d". The infrared (IR) spectra were determined as neat oils. $R_{f}$ values indicated refer to thin-layer chromatography on $2.5 \times 10 \mathrm{~cm}, 250-\mu \mathrm{m}$ analytical plates coated with silica gel GF and developed in the solvent indicated. Flash column chromatography was performed with Merck 35-60 $\mu$ silica gel following the procedure of Still. ${ }^{1}$ Where specified, TLC mesh silica gel was employed, following the procedure described by Taber. ${ }^{2}$ Solvents are referred as volume/volume mixture. PE is petroleum ether. EtOAc is ethyl acetate. MTBE is methyl tert-butyl ether. THF and diethyl ether were distilled from sodium/benzophenone under $\mathrm{N}_{2}$ and used immediately. $\mathrm{CH}_{2} \mathrm{Cl}_{2}$ was distilled from $\mathrm{CaH}_{2}$ under $\mathrm{N}_{2}$. All reactions were carried out in flame-dried glassware under a positive pressure of $\mathrm{N}_{2}$ unless otherwise indicated. The alkenes were prepared by adding the corresponding ketone to the appropriate Grignard reagent or alkynyl lithium, followed by $p$-toluenesulfonic acid-catalyzed dehydration of the product alcohol. ${ }^{3}$ Known epoxides were prepared according to the literaure.

\section{General procedure for preparation of epoxides $(1 \mathbf{a}, 1 \mathbf{b}, 1 \mathrm{c})$}

The alkene $(10 \mathrm{mmol}), \mathrm{CH}_{2} \mathrm{Cl}_{2}(80 \mathrm{~mL})$ and saturated aqueous $\mathrm{NaHCO}_{3}(80 \mathrm{~mL})$ were combined, and the mixture was cooled in an ice water bath. mCPBA $(70 \%, 2.70 \mathrm{~g}, 11.0$ mmol) was added, and the resulting mixture was stirred vigorously at $0{ }^{\circ} \mathrm{C}$ for $1-2 \mathrm{~h}$ with monitoring by TLC. The layers were separated and the organic layer was partitioned between $\mathrm{CH}_{2} \mathrm{Cl}_{2}$ and, sequentially, $1 \mathrm{~N}$ aqueous $\mathrm{NaOH}$ and saturated aqueous $\mathrm{NaCl}$. The 
combined organic extract was dried $\left(\mathrm{Na}_{2} \mathrm{SO}_{4}\right)$ and concentrated, and the residue was chromatographed.

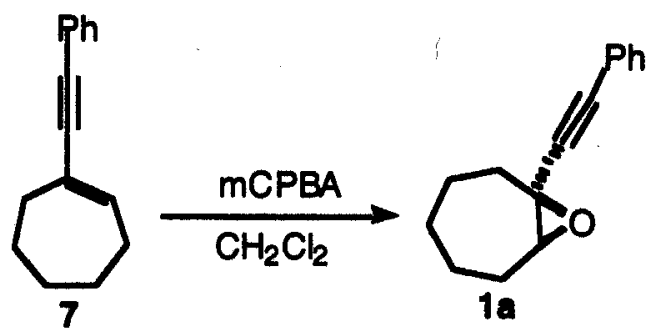

\section{Epoxide 1a}

Clear oil (91\% yield), TLC $R_{f}=0.71$ (PE: MTBE, 9:1). IR (neat, $\mathrm{cm}^{-1}$ ): $2928(\mathrm{~s}), 2852$ (m), 1598 (w), 1491 (m), 1443 (m); ${ }^{1} \mathrm{H}$ NMR (400 MHz, $\left.\mathrm{CDCl}_{3}\right)$ 8 7.25-7.45 (m, 5H), $3.38(\mathrm{dd}, \mathrm{J}=6.8,3.4 \mathrm{~Hz}, 1 \mathrm{H}), 1.37-2.30(\mathrm{~m}, 10 \mathrm{H}) ;{ }^{13} \mathrm{C} \mathrm{NMR}\left(\mathrm{CDCl}_{3}, 100 \mathrm{MHz}\right) \delta \mathrm{u}$ : $122.6,90.6,81.5,54.7,34.7,31.3,29.4,24.9,24.4 ; \mathrm{d}: 132.0,128.6,128.4,64.0 ;$ HRMS calcd for $\mathrm{C}_{15} \mathrm{H}_{16} \mathrm{O}\left(\mathrm{M}^{+}\right)$212.1201, found 212.1195.

\section{Typical procedure for the ring opening of epoxides with Grignard reagents}

To a solution of epoxide $(2 \mathrm{mmol})$ in anhydrous THF $(15 \mathrm{~mL})$ was added dropwise a solution of Grignard reagent ( $8 \mathrm{mmol}$, if indicated, $10 \mathrm{mmol}$ was used in some cases) over two minutes at $0^{\circ} \mathrm{C}$. The reaction mixture was warmed slowly to room temperature, then stirred overnight. The reaction mixture was partitioned between saturated aqueous $\mathrm{NH}_{4} \mathrm{Cl}$ and ether. The combined organic extract was dried $\left(\mathrm{Na}_{2} \mathrm{SO}_{4}\right)$ and concentrated, and the residue was chromatographed. 


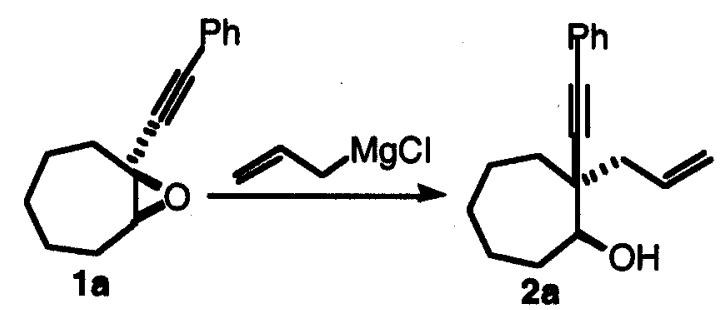

\section{Alcohol 2a}

Clear oil (83\% yield), TLC $R_{f}=0.64$ (PE: acetone, 3:1). IR (neat, $\mathrm{cm}^{-1}$ ): 3434 (s), 2932 (s), 2240 (w), 1638 (w), 1597 (w), 1490 (m), 1442 (m); ${ }^{1} \mathrm{H}$ NMR (400 MHz, $\mathrm{CDCl}_{3}$ ) $\delta$ 7.26-7.32 (m, 5H), $6.00(\mathrm{~m}, 1 \mathrm{H}), 5.14(\mathrm{~d}, \mathrm{~J}=11.6 \mathrm{~Hz}, 1 \mathrm{H}), 5.13(\mathrm{~d}, \mathrm{~J}=15.2 \mathrm{~Hz}, 1 \mathrm{H}), 3.36$ $(\mathrm{dd}, \mathrm{J}=9.2,4.0 \mathrm{~Hz}, 1 \mathrm{H}), 1.30-2.80(\mathrm{~m}, 13 \mathrm{H}) ;{ }^{13} \mathrm{C} \mathrm{NMR}\left(\mathrm{CDCl}_{3}, 100 \mathrm{MHz}\right) \delta \mathrm{u}: 123.5$, $118.2,91.5,86.1,47.1,44.4,34.6,33.7,27.0,23.0,22.1$; d: 135.2, 131.9, 128.4, 128.2, 78.3; HRMS calcd for $\mathrm{C}_{18} \mathrm{H}_{23} \mathrm{O}(\mathrm{M}+\mathrm{H})$ 255.1749, found 255.1743.

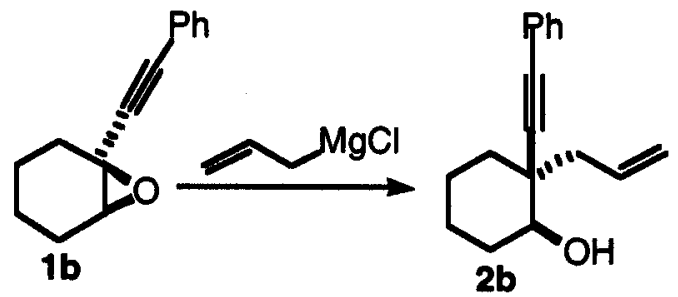

\section{Alcohol 2b}

Clear oil (88\% yield), TLC $R_{f}=0.66$ (PE: MTBE, 1:1). IR (neat, $\mathrm{cm}^{-1}$ ): 3399 (s), 2933 (s), 2240 (w), 1639 (w), 1597 (w), 1490 (m), 1443 (m), 1050 (m); ${ }^{1} \mathrm{H}$ NMR (400 MHz, $\left.\mathrm{CDCl}_{3}\right) \delta$ 7.25-7.43 (m, 5H), $6.00(\mathrm{~m}, 1 \mathrm{H}), 5.14(\mathrm{~d}, \mathrm{~J}=16.8 \mathrm{~Hz}, 1 \mathrm{H}), 5.13(\mathrm{~d}, \mathrm{~J}=9.6 \mathrm{~Hz}$, $1 \mathrm{H}), 3.30(\mathrm{br}, 1 \mathrm{H}), 1.10-2.80(\mathrm{~m}, 11 \mathrm{H}) ;{ }^{13} \mathrm{C} \mathrm{NMR}\left(\mathrm{CDCl}_{3}, 100 \mathrm{MHz}\right) \delta \mathrm{u}: 123.6,118.1$, $91.1,86.4,44.8,43.7,35.5,32.8,24.9,22.5$; d: $134.9,131.9,128.4,128.1,75.3 ;$ HRMS calcd for $\mathrm{C}_{17} \mathrm{H}_{21} \mathrm{O}(\mathrm{M}+\mathrm{H}) 241.1592$, found 241.1582. 


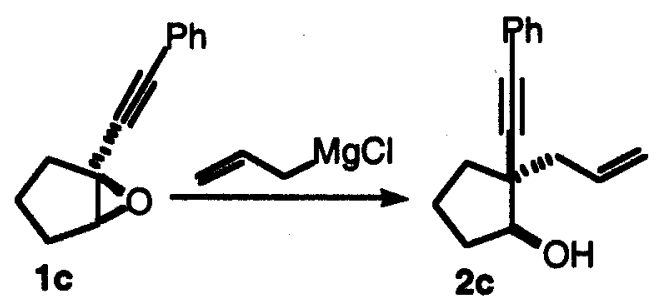

\section{Alcohol 2c}

Clear oil (88\% yield), TLC $R_{f}=0.58$ (PE: MTBE, 1:1). IR (neat, $\mathrm{cm}^{-1}$ ): 3448 (s), 2965 (s), 2238 (w), 1640 (w), 1598 (w), 1490 (s), 1443 (m), 1092 (s), 914 (s); ${ }^{1}$ H NMR (400 $\left.\mathrm{MHz}, \mathrm{CDCl}_{3}\right)$ 8 7.25-7.42 (m, 5H), $6.03(\mathrm{~m}, 1 \mathrm{H}), 5.16(\mathrm{~m}, 1 \mathrm{H}), 5.11(\mathrm{~m}, 1 \mathrm{H}), 3.85(\mathrm{dd}$, $\mathrm{J}=11.2 \mathrm{~Hz}, 5.6 \mathrm{~Hz}, 1 \mathrm{H}), 1.55-2.44(\mathrm{~m}, 9 \mathrm{H}) ;{ }^{13} \mathrm{C} \mathrm{NMR}\left(\mathrm{CDCl}_{3}, 100 \mathrm{MHz}\right) \delta \mathrm{u}: 123.3$, $117.7,91.2,85.9,49.0,42.5,35.6,32.2,20.4 ;$ d: 135.0, 131.9, 128.4, 128.2, 79.2; HRMS calcd for $\mathrm{C}_{16} \mathrm{H}_{19} \mathrm{O}(\mathrm{M}+\mathrm{H})$ 227.1436, found 227.1434.

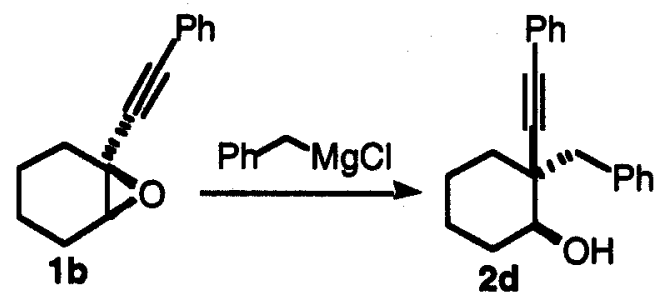

\section{Alcohol 2d}

Clear oil (84\% yield), TLC $R_{f}=0.34$ (PE:MTBE, 4:1). IR (neat, $\mathrm{cm}^{-1}$ ): 3426 (s), 2932 (s), 2856 (m), 1491 (m), 1444 (m); ${ }^{1} \mathrm{H}$ NMR (400 MHz, $\mathrm{CDCl}_{3}$ ) 8 7.20-7.43 (m, 10H), $3.31(\mathrm{dt}, \mathrm{J}=6.4 \mathrm{~Hz}, 10.4 \mathrm{~Hz}, 1 \mathrm{H}), 3.27$ (d, J=13.0 Hz, 1H), $2.83(\mathrm{~d}, \mathrm{~J}=13.0 \mathrm{~Hz}, 1 \mathrm{H}), 1.17-$ $1.92(\mathrm{~m}, 9 \mathrm{H}) ;{ }^{13} \mathrm{C} \mathrm{NMR}\left(\mathrm{CDCl}_{3}, 100 \mathrm{MHz}\right) \delta \mathrm{u}: 137.4,123.5,91.3,87.1,45.9,44.8,35.0$, 33.0, 24.8, 22.4; d: 131.8, 131.1, 128.4, 128.1, 127.9, 126.5, 74.8; HRMS calcd for $\mathrm{C}_{21} \mathrm{H}_{22} \mathrm{O}\left(\mathrm{M}^{+}\right)$290.1671, found 290.1683. 


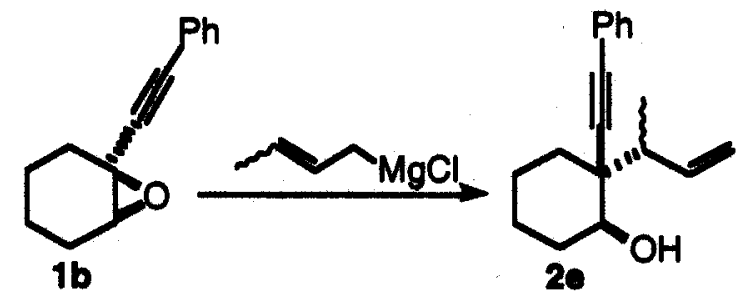

Alcohol 2e (2:1 ratio of two isomers)

Clear oil (85\% yield), TLC $R_{f}=0.42$ (PE:MTBE, 4:1). IR (neat, $\mathrm{cm}^{-1}$ ): 3442 (s), 2933 (s), 2857 (m), 1490 (m), 1444 (m), 1052 (m); ${ }^{1} \mathrm{H}$ NMR (400 MHz, $\mathrm{CDCl}_{3}$ ) $\delta$ major isomer: 7.23-7.44 (m, $5 \mathrm{H}), 5.80(\mathrm{~m}, 1 \mathrm{H}), \mathbf{5 . 1 0 - 5 . 2 0}(\mathrm{m}, 2 \mathrm{H}), 3.49(\mathrm{~m}, 1 \mathrm{H}), 2.88(\mathrm{~m}, 1 \mathrm{H})$, 1.50-1.91 (m, 9H), $1.21(\mathrm{~d}, \mathrm{~J}=6.8 \mathrm{~Hz}, 3 \mathrm{H})$; minor isomer: 7.23-7.44 (m, 5H), $6.15(\mathrm{~m}$, $1 \mathrm{H}), 5.01-5.14(\mathrm{~m}, 2 \mathrm{H}), 3.55(\mathrm{~m}, 1 \mathrm{H}), 2.68(\mathrm{~m}, 1 \mathrm{H}), 1.50-1.91(\mathrm{~m}, 9 \mathrm{H}), 1.12(\mathrm{~d}, \mathrm{~J}=6.8$ $\mathrm{Hz}, 3 \mathrm{H}) ;{ }^{13} \mathrm{C} \mathrm{NMR}\left(\mathrm{CDCl}_{3}, 100 \mathrm{MHz}\right) \delta$ (two isomers are reported together): $\mathrm{u}: 123.6$, $116.7,114.9,113.5,91.6,91.3,86.9,86.2,48.3,47.8,32.9,32.3,31.0,29.4,24.6,24.4$, $22.4,22.3 ;$ d: $142.0,139.4,131.8,131.7,128.4,128.3,128.0,127.9,73.0,72.4,43.5$, 42.5, 16.1, 14.4; HRMS calcd for $\mathrm{C}_{18} \mathrm{H}_{22} \mathrm{O}\left(\mathrm{M}^{+}\right)$254.1671, found 254.1659. 


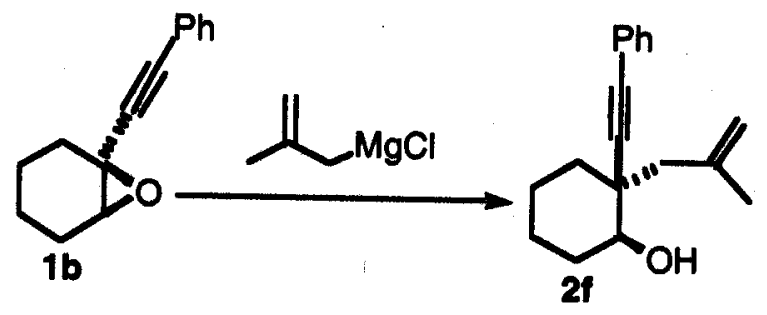

\section{Alcohol $2 f$}

Clear oil (76\% yield), TLC $R_{f}=0.36$ (PE:MTBE, 4:1). IR (neat, $\mathrm{cm}^{-1}$ ): $3406(\mathrm{~s}), 2933$ (s), 2856 (m), 1643 (w), 1490 (m), 1443 (m), 1044 (m); ${ }^{1} \mathrm{H}$ NMR (400 MHz, $\mathrm{CDCl}_{3}$ ) $\delta$ 7.39-7.43 (m, 2H), 7.26-7.31 (m, 3H), $4.91(\mathrm{~m}, 1 \mathrm{H}), 4.82(\mathrm{~m}, 1 \mathrm{H}), 3.29(\mathrm{dt}, \mathrm{J}=4.0 \mathrm{~Hz}$, $10.0 \mathrm{~Hz}, 1 \mathrm{H}), 2.78$ (d, J=13.2 Hz, 1H), 2.19 (d, J=13.2 Hz, 1H), $1.96(\mathrm{~s}, 3 \mathrm{H}), 1.16-2.00$ $(\mathrm{m}, 9 \mathrm{H}) ;{ }^{13} \mathrm{C} \mathrm{NMR}\left(\mathrm{CDCl}_{3}, 100 \mathrm{MHz}\right) \delta \mathrm{u}: 143.1,123.6,115.0,91.8,86.5,47.5,44.1$, 35.6, 32.6, 24.8, 22.5; d: 131.7, 128.4, 128.0, 76.4, 24.7; HRMS calcd for $\mathrm{C}_{18} \mathrm{H}_{22} \mathrm{O}\left(\mathrm{M}^{+}\right)$ 254.1671 , found 254.1675 .

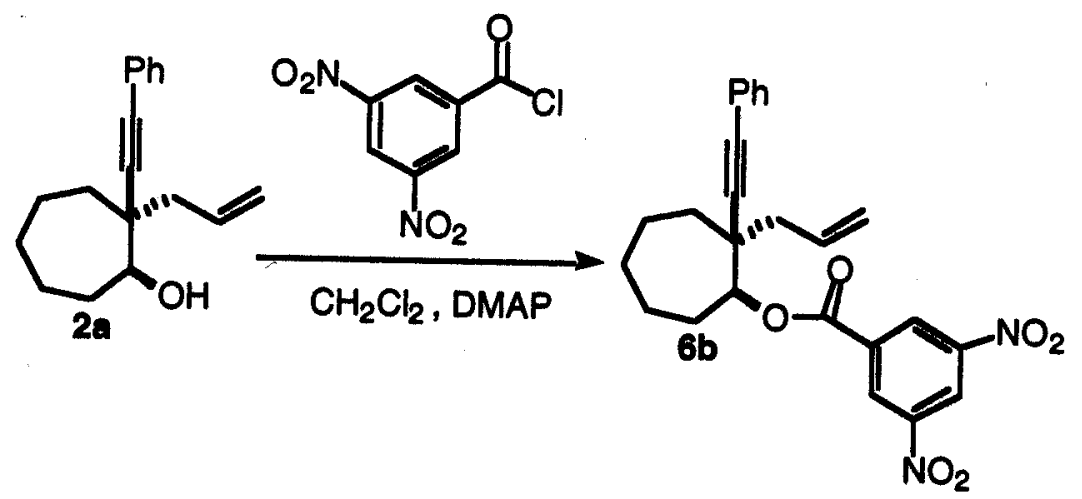

\section{Benzoate 6b}

A solution of alcohol $2 \mathrm{a}(0.226 \mathrm{~g}, 0.890 \mathrm{mmol})$ in $10 \mathrm{~mL}$ of anhydrous $\mathrm{CH}_{2} \mathrm{Cl}_{2}$ was added DMAP $(0.217 \mathrm{~g}, 1.780 \mathrm{mmol})$ and 3,5-dinitrobenzoyl chloride $(0.410 \mathrm{~g}, 1.780 \mathrm{mmol})$ sequentially at room temperature. The resulting mixture was stirred at room temperature 
for $3 \mathrm{~h}$, then partitioned between $\mathrm{CH}_{2} \mathrm{Cl}_{2}$ and, sequentially, saturated aqueous $\mathrm{NH}_{4} \mathrm{Cl}$ and saturated aqueous $\mathrm{NaCl}$. The $\mathrm{CH}_{2} \mathrm{Cl}_{2}$ extract was dried $\left(\mathrm{Na}_{2} \mathrm{SO}_{4}\right)$ and concentrated, and the residue was chromatographed to give $0.386 \mathrm{~g}$ of benzoate $6 \mathrm{~b}$ ( $97 \%$ yield) as colorless crystals: $\mathrm{mp}=113-114^{\circ} \mathrm{C}$, TLC $R_{f}=0.55$ (PE: MTBE, 5:1). IR (film, $\mathrm{cm}^{-1}$ ): 2937 (m), 1728 (s), 1628 (w), 1548 (s), 1344 (s), 1276 (s), 1170 (m); ${ }^{1} \mathrm{H}$ NMR (400 MHz, $\left.\mathrm{CDCl}_{3}\right) \delta$ $9.23(\mathrm{~s}, 3 \mathrm{H}), 7.33-7.56(\mathrm{~m}, 5 \mathrm{H}), 5.97(\mathrm{~m}, 1 \mathrm{H}), 5.07(\mathrm{~s}, 1 \mathrm{H}), 4.98-5.04(\mathrm{~m}, 2 \mathrm{H}), 1.61-2.53$ $(\mathrm{m}, 12 \mathrm{H}) ;{ }^{13} \mathrm{C} \mathrm{NMR}\left(\mathrm{CDCl}_{3}, 100 \mathrm{MHz}\right) \delta \mathrm{u}: 161.9,148.9,134.5,123.5,118.7,90.9,85.3$, $45.0,44.5,34.7,31.2,26.7,22.6,22.1 ;$ d: $134.0,132.0,129.7,128.6,128.3,122.6,82.6$; HRMS calcd for $\mathrm{C}_{25} \mathrm{H}_{24} \mathrm{~N}_{2} \mathrm{O}_{6}\left(\mathrm{M}^{+}\right)$448.1634, found 448.1646.

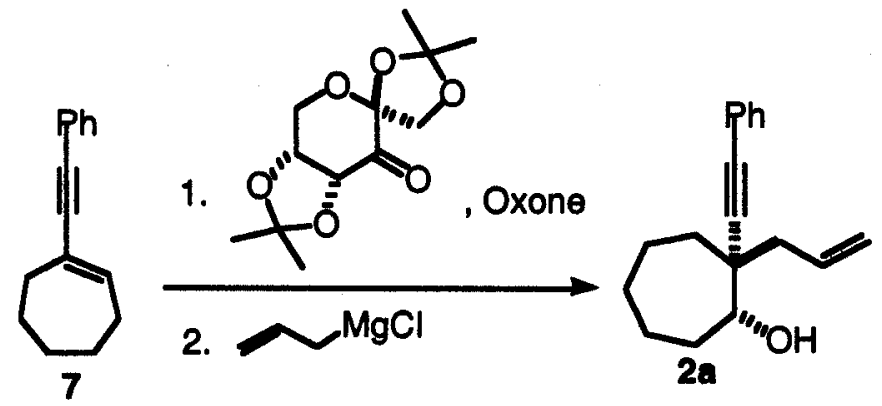

\section{(+)-Alcohol 2a}

To a $500 \mathrm{~mL}$ three -necked flask was added alkene $8(2.94 \mathrm{~g}, 15.0 \mathrm{mmol}), 150 \mathrm{~mL}$ of a 2:1 mixture of dimethoxymethane and acetonitrile, $90 \mathrm{~mL}$ of potassium carbonate-acetic acid buffer solution, ${ }^{4}$ tetrabutylammonium hydrogen sulfate $(102 \mathrm{mg})$ and Shi's chiral ketone $^{3}(1.35 \mathrm{~g}, 2.93 \mathrm{mmol})$. The flask was connected to two dropping funnels, one charged with a solution of oxone $(13.8 \mathrm{~g}, 22.5 \mathrm{mmol})$ in $51 \mathrm{~mL}$ of aqueous $4 \times 10^{-4}$ $\mathrm{Na}_{2} \mathrm{EDTA}$ and the other charged with $51 \mathrm{~mL}$ of $1.47 \mathrm{M}$ aqueous $\mathrm{KOH}$. The two solutions were added dropwise at the same rate over $70 \mathrm{~min}$ to the cold reaction mixture which was stirred vigorously at $0^{\circ} \mathrm{C}$. After $2 \mathrm{~h}$, ether $(150 \mathrm{~mL})$ was added. The biphasic mixture was 
partitioned between ether and, sequentially, water and brine. The organic extract was dried $\left(\mathrm{Na}_{2} \mathrm{SO}_{4}\right)$ and concentrated. The residue was dissolved in $50 \mathrm{~mL}$ of anhydrous THF. To this solution allylmagnesium chloride $(32.6 \mathrm{~mL}, 2.0 \mathrm{M})$ was added dropwise over 10 min at $0{ }^{\circ} \mathrm{C}$ under $\mathrm{N}_{2}$. The reaction solution was warmed slowly from $0{ }^{\circ} \mathrm{C}$ to room temperature, then maintained at ambient temperature overnight. The reaction mixture was partitioned between saturated aqueous $\mathrm{NH}_{4} \mathrm{Cl}$ and ether. The combined organic extract was dried over $\mathrm{Na}_{2} \mathrm{SO}_{4}$ and concentrated in vacuo. The residue was chromatographed to give $2.74 \mathrm{~g}$ of alcohol $2 \mathrm{a}$ (72\% yield from 7) as a colorless oil, TLC $R_{f}=0.64$ (PE:

acetone, 3:1)., $[\alpha]_{D}^{22}=+63.1$ (c 1.7, THF); IR (neat, $\mathrm{cm}^{-1}$ ): 3434 (s), 2932 (s), $2240(\mathrm{w})$, 1638 (w), 1597 (w), 1490 (m), 1442 (m); ${ }^{1} \mathrm{H}$ NMR (400 MHz, CDCl $\left.)_{3}\right)$ 8 7.26-7.32 (m, $5 \mathrm{H}), 6.00(\mathrm{~m}, 1 \mathrm{H}), 5.14(\mathrm{~d}, \mathrm{~J}=11.6 \mathrm{~Hz}, 1 \mathrm{H}), 5.13(\mathrm{~d}, \mathrm{~J}=15.2 \mathrm{~Hz}, 1 \mathrm{H}), 3.36(\mathrm{dd}, \mathrm{J}=9.2$, $4.0 \mathrm{~Hz}, 1 \mathrm{H}), 1.30-2.80(\mathrm{~m}, 13 \mathrm{H}) ;{ }^{13} \mathrm{C} \mathrm{NMR}\left(\mathrm{CDCl}_{3}, 100 \mathrm{MHz}\right) \delta \mathrm{u}: 123.5,118.2,91.5$, $86.1,47.1,44.4,34.6,33.7,27.0,23.0,22.1 ;$ d: $135.2,131.9,128.4,128.2,78.3 ;$ HRMS calcd for $\mathrm{C}_{18} \mathrm{H}_{23} \mathrm{O}(\mathrm{M}+\mathrm{H}) 255.1749$, found 255.1743 .

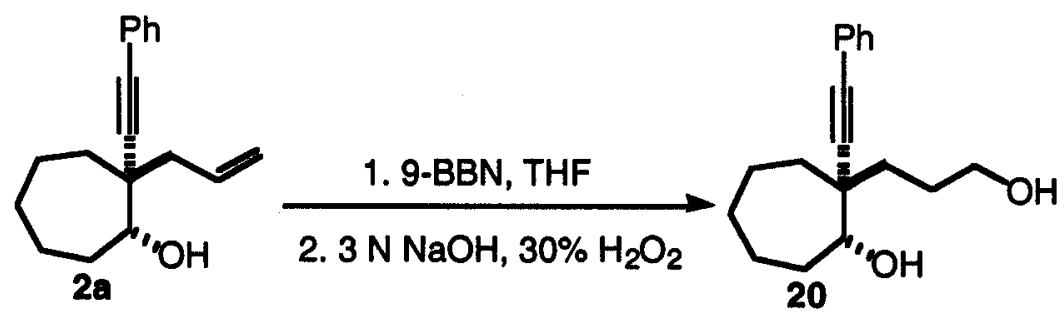

\section{(+)-Diol 20}

To alcohol $2 \mathrm{a}(2.70 \mathrm{~g}, 10.6 \mathrm{mmol})$ was added 9-BBN in THF $(0.5 \mathrm{M}, 53.2 \mathrm{~mL})$ slowly over 10 min under $\mathrm{N}_{2}$ and stirring at $0{ }^{\circ} \mathrm{C}$. The resulting solution was stirred at ambient temperature overnight. The reaction mixture was cooled in an ice water bath and aqueous 
$\mathrm{NaOH}$ solution $(3 \mathrm{~N}, 15 \mathrm{~mL})$ was added, followed by addition of $30 \% \mathrm{H}_{2} \mathrm{O}_{2}(15 \mathrm{~mL})$ very carefully. After stirring at room temperature for $3 \mathrm{~h}$, the reaction mixture was partitioned between brine and ether. The combined organic extract was dried over $\mathrm{Na}_{2} \mathrm{SO}_{4}$ and concentrated in vacuo. The residue was chromatographed to give $2.45 \mathrm{~g}$ of diol 21 (85\% yield) as a colorless oil, TLC $R_{f}=0.5$ (ether), $[\alpha]^{22}{ }_{\mathrm{D}}=+5.61\left(\mathrm{c} 0.82\right.$, THF). IR (neat, $\mathrm{cm}^{\text {* }}$ $\left.{ }^{1}\right): 3362$ (s), 2933 (s), 2224 (w), 1490 (m), 1442 (m), 1052 (s); ${ }^{1} \mathrm{H}$ NMR (400 MHz, $\left.\mathrm{CDCl}_{3}\right)$ 8 7.38-7.43 (m, 2H), 7.25-7.30 (m, 3H), 3.60-3.70 (m, 2H), 3.34 (dd, J=9.2 Hz, $3.0 \mathrm{~Hz}, 1 \mathrm{H}), 2.89(\mathrm{br}, 1 \mathrm{H}), 2.60(\mathrm{br}, 1 \mathrm{H}), 1.34-1.92(\mathrm{~m}, 14 \mathrm{H}) ;{ }^{13} \mathrm{C} \mathrm{NMR}\left(\mathrm{CDCl}_{3}, 100\right.$ $\mathrm{MHz}) \delta \mathrm{u}: 123.5,92.0,85.7,63.1,46.6,35.8,34.4,33.8,28.0,26.9,22.9,22.2 ;$ d: 131.8 , 128.3, 128.0, 78.2; HRMS calcd for $\mathrm{C}_{18} \mathrm{H}_{24} \mathrm{O}_{2}\left(\mathrm{M}^{+}\right)$272.1776, found 272.1776.

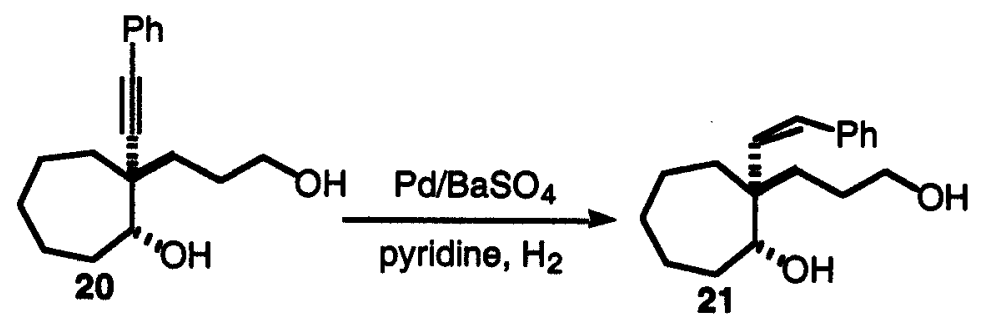

\section{(·)-Diol 21}

Diol $20(1.73 \mathrm{~g}, 6.36 \mathrm{mmol})$ in pyridine $(20 \mathrm{~mL})$ was added palladium on $\mathrm{BaSO}_{4}(677$ $\mathrm{mg}$ ). The resulting mixture then stirred under $\mathrm{H}_{2}$ atmosphere ( $\left.1 \mathrm{~atm}\right)$ for $23 \mathrm{~h}$. Silica gel $(4.0 \mathrm{~g})$ was added and the mixture was concentrated. The residual solid was chromatographed to give $1.65 \mathrm{~g}$ of diol 21 (95\% yield) as a colorless oil, TLC $R_{f}=0.5$ (ether), $[\alpha]^{22}=-40.9(\mathrm{c} 0.85, \mathrm{THF}) . \mathrm{IR}$ (neat, $\mathrm{cm}^{-1}$ ): $3393(\mathrm{~s}), 2924(\mathrm{~s}), 1458(\mathrm{~m}), 1030$ (m); ${ }^{1} \mathrm{H} \mathrm{NMR}\left(400 \mathrm{MHz}, \mathrm{CDCl}_{3}\right)$ 8 7.17-7.28 (m, 5H), $6.65(\mathrm{~d}, \mathrm{~J}=13.2 \mathrm{~Hz}, 1 \mathrm{H}), 5.75$ (d, $\mathrm{J}=13.2 \mathrm{~Hz}, 1 \mathrm{H}), 3.55(\mathrm{~d}, \mathrm{~J}=8.4 \mathrm{~Hz}, 1 \mathrm{H}), 3.38-3.44(\mathrm{~m}, 2 \mathrm{H}), 3.29(\mathrm{~s}, 1 \mathrm{H}), 3.13(\mathrm{~s}, 1 \mathrm{H})$, 
1.29-1.83 (m, 14H); ${ }^{13} \mathrm{C} \mathrm{NMR}\left(\mathrm{CDCl}_{3}, 100 \mathrm{MHz}\right) \delta \mathrm{u}: 139.4,63.1,48.1,34.9,34.2,32.9$, 27.6, 27.1, 23.0, 22.1; d: 136.3, 130.4, 128.4, 127.7, 126.5, 77.6; HRMS calcd for $\mathrm{C}_{18} \mathrm{H}_{26} \mathrm{O}_{2}\left(\mathrm{M}^{+}\right)$274.1933, found 274.1919.

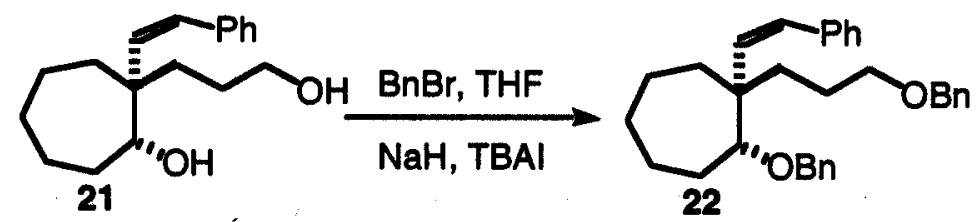

\section{$(-)$-Benzyl ether 22}

To a stirred solution of diol $21(1.34 \mathrm{~g}, 4.89 \mathrm{mmol})$ in dry THF ( $25 \mathrm{~mL})$ was added tetrabutylammonium bromide (18 mg, $0.049 \mathrm{mmol})$ and 60\% $\mathrm{NaH}(783 \mathrm{mg}, 19.6 \mathrm{mmol})$. After stirring at room temperature under $\mathrm{N}_{2}$ for $30 \mathrm{~min}, \mathrm{BnBr}(1.45 \mathrm{~mL}, 12.2 \mathrm{mmol})$ was added. The resulting mixture was refluxed gently under $\mathrm{N}_{2}$ for $8 \mathrm{~h}$. The reaction was partitioned between water and ether. The combined organic extract was dried over $\mathrm{Na}_{2} \mathrm{SO}_{4}$ and concentrated in vacuo. The residue was chromatographed to give $1.89 \mathrm{~g}$ of benzyl ether 22 (85\% yield) as a colorless oil, TLC $R_{f}=0.73$ (PE:MTBE, 9:1), $[\alpha]_{\mathrm{D}}^{22}=-$ 25.9 (c 0.93, THF). IR (neat, $\mathrm{cm}^{-1}$ ): 2929 (s), 2857 (s), 1494 (m), 1453 (s), 1361 (m), 1098 (s), 1070 (s); ${ }^{1} \mathrm{H}$ NMR (400 MHz, $\mathrm{CDCl}_{3}$ ) $\delta$ 7.13-7.33 (m, 15H), 6.57 (d, J= 13.2 $\mathrm{Hz}, 1 \mathrm{H}), 5.85(\mathrm{~d}, \mathrm{~J}=13.2 \mathrm{~Hz}, 1 \mathrm{H}), 4.63(\mathrm{~d}, \mathrm{~J}=12.0 \mathrm{~Hz}, 1 \mathrm{H}), 4.40(\mathrm{~s}, 2 \mathrm{H}), 4.36(\mathrm{~d}, \mathrm{~J}=12.0$ $\mathrm{Hz}, 1 \mathrm{H}), 3.25(\mathrm{~d}, \mathrm{~J}=8.0 \mathrm{~Hz}, 1 \mathrm{H}), 3.18-3.21(\mathrm{~m}, 2 \mathrm{H}), 1.12-1.81(\mathrm{~m}, 14 \mathrm{H}) ;{ }^{13} \mathrm{C}$ NMR $\left(\mathrm{CDCl}_{3}, 100 \mathrm{MHz}\right) \delta \mathrm{u}: 140.0,139.3,138.9,72.9,71.4,71.0,48.3,35.2,34.9,28.5,28.0$, $25.0,24.3,22.4$; d: $137.6,129.0,128.7,128.5,128.4,127.8,127.7,127.6,127.5,127.4$, 126.3, 86.0; HRMS calcd for $\mathrm{C}_{32} \mathrm{H}_{38} \mathrm{O}_{2} \mathrm{Na}(\mathrm{M}+\mathrm{Na})$ 477.2770, found 477.2774. 


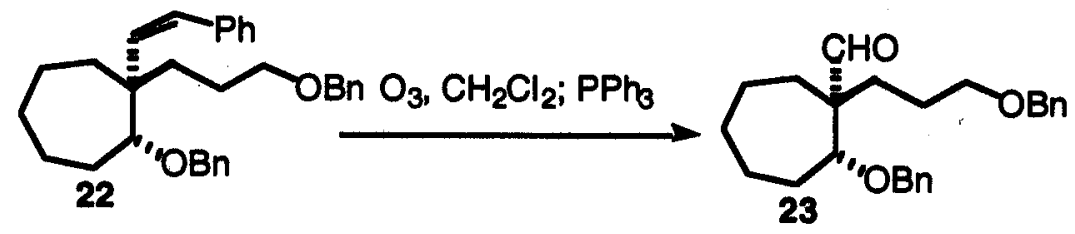

\section{(-)-Aldehyde 23}

A solution of alkene $22(1.60 \mathrm{~g}, 3.52 \mathrm{mmol})$ in $\mathrm{CH}_{2} \mathrm{Cl}_{2}(50 \mathrm{~mL})$ was cooled to $-78^{\circ} \mathrm{C}$. After purging with $\mathrm{O}_{2}$ for $2 \mathrm{~min}, \mathrm{O}_{3}$ was bubbled through the solution until the pale bule color persisted. The reaction was purged with $\mathrm{N}_{2}$ for $5 \mathrm{~min}$, then $\mathrm{PPh}_{3}(1.38 \mathrm{~g}, 5.28$ mmol) was added. The resuling solution was first stirred at $-78^{\circ} \mathrm{C}$ for $1 \mathrm{~h}$, then room temperature for $1 \mathrm{~h}$. Silica gel $(4.0 \mathrm{~g})$ was added and the mixture was concentrated. The residual solid was chromatographed to give $1.18 \mathrm{~g}$ of aldehyde 23 ( $88 \%$ yield) as a colorless oil, TLC $R_{f}=0.54$ (PE:MTBE, 4:1), $[\alpha]_{\mathrm{D}}^{22}=-14.0$ (c 0.8, THF). IR (neat, $\mathrm{cm}^{*}$ '): 2967 (s), 2858 (s), 1726 (s), 1453 (m), 1092 (s); ${ }^{1} \mathrm{H}$ NMR (400 MHz, CDCl 3 ) $\delta 9.54$ (s, 1H), 7.25-7.36 (m, 10H), $4.60(\mathrm{~d}, \mathrm{~J}=11.6 \mathrm{~Hz}, 1 \mathrm{H}), 4.46(\mathrm{~s}, 2 \mathrm{H}), 4.30(\mathrm{~d}, \mathrm{~J}=11.6 \mathrm{~Hz}, 1 \mathrm{H})$, $3.66(\mathrm{~d}, \mathrm{~J}=7.2 \mathrm{~Hz}, 1 \mathrm{H}), 3.38-3.43(\mathrm{~m}, 2 \mathrm{H}), 1.36-2.04(\mathrm{~m}, 14 \mathrm{H}) ;{ }^{13} \mathrm{C} \mathrm{NMR}\left(\mathrm{CDCl}_{3}, 100\right.$ MHz) $\delta$ u: $138.6,138.4,73.0,70.8,70.6,56.3,30.3,29.3,27.8,27.2,24.3,22.8,22.1$; : 205.6, 128.6, 128.5, 127.8, 127.7, 127.6, 127.5, 83.0; HRMS calcd for $\mathrm{C}_{25} \mathrm{H}_{32} \mathrm{O}_{3} \mathrm{Na}$ $(\mathrm{M}+\mathrm{Na})$ 403.2249, found 403.2251. 


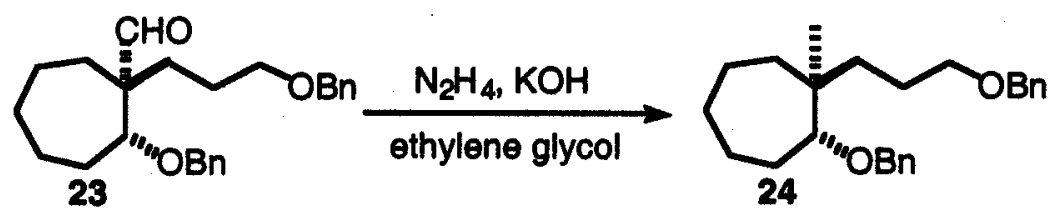

\section{(-)-Benzyl ether 24}

To a $25 \mathrm{~mL}$ round bottom flask was added aldehyde $23(424 \mathrm{mg}, 1.12 \mathrm{mmol})$, ethylene glycol (10 mL), $\mathrm{N}_{2} \mathrm{H}_{4}(64 \%, 718 \mathrm{mg}, 22.4 \mathrm{mmol})$ and ground $\mathrm{KOH}(86 \%, 1.46 \mathrm{~g}, 22.4$ mmol). The flask was connected to a short-path distillation head. The reaction mixture was heated at $130^{\circ} \mathrm{C}$ under $\mathrm{N}_{2}$ for $2 \mathrm{~h}$, then $210^{\circ} \mathrm{C}$ for $3 \mathrm{~h}$. After cooling down, the reaction mixture was partitioned between water and $\mathrm{CH}_{2} \mathrm{Cl}_{2}$. The combined organic extract was dried over $\mathrm{Na}_{2} \mathrm{SO}_{4}$ and concentrated in vacuo. The residue was chromatographed to give $365 \mathrm{mg}$ of benzyl ether 24 ( $89 \%$ yield) as a colorless oil, TLC $R_{f}=0.67$ (PE:MTBE, 9:1), $[\alpha]_{\mathrm{D}}^{22}=-31.6(\mathrm{c} 0.7, \mathrm{THF})$. IR (neat, $\mathrm{cm}^{-1}$ ): 2927 (s), 2854 (s), 1453 (m), 1098 (s); ${ }^{1} \mathrm{H}$ NMR (400 MHz, $\mathrm{CDCl}_{3}$ ) 8 7.22-7.34 (m, 10H), 4.59 (d, $\mathrm{J}=11.6 \mathrm{~Hz}, 1 \mathrm{H}), 4.47(\mathrm{~s}, 2 \mathrm{H}), 4.31(\mathrm{~d}, \mathrm{~J}=11.6 \mathrm{~Hz}, 1 \mathrm{H}), 3.39$ (dd, J=7.6 Hz, $6.4 \mathrm{~Hz}, 1 \mathrm{H})$, 3.39 (dd, J= 6.4 Hz, $5.6 \mathrm{~Hz}, 1 \mathrm{H}), 3.11$ (dd, J=6.8 Hz, $3.8 \mathrm{~Hz}, 1 \mathrm{H}), 1.27-1.74(\mathrm{~m}, 14 \mathrm{H})$, $0.91(\mathrm{~s}, 3 \mathrm{H}) ;{ }^{13} \mathrm{C} \mathrm{NMR}\left(\mathrm{CDCl}_{3}, 100 \mathrm{MHz}\right) \delta \mathrm{u}: 139.5,138.8,73.0,71.6,71.3,40.2,36.8$, $36.2,29.6,27.6,25.5,24.5,22.3 ;$ d: $128.5,128.3,127.7,127.6,127.5,127.3,86.5,22.1$; HRMS calcd for $\mathrm{C}_{25} \mathrm{H}_{35} \mathrm{O}_{2}(\mathrm{M}+\mathrm{H})$ 367.2637, found 367.2629. 


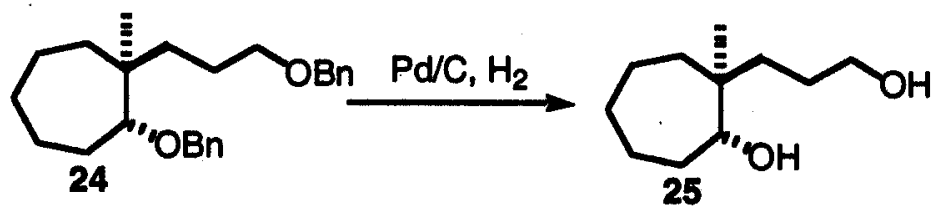

\section{(+)-Diol 25}

To a solution of benzyl ether $24(180 \mathrm{mg}, 0.492 \mathrm{mmol})$ in absolute ethanol $(5 \mathrm{~mL})$ was added $10 \% \mathrm{Pd} / \mathrm{C}(20 \mathrm{mg})$. The mixture was stirred under $\mathrm{H}_{2}$ atmosphere $(1 \mathrm{~atm})$ for $15 \mathrm{~h}$. Silica gel $(1.0 \mathrm{~g})$ was added and the mixture was concentrated. The residual solid was chromatographed to give $84 \mathrm{mg}$ of diol 25 (92\% yield) as a colorless oil, TLC $R_{f}=0.44$ (ether), $[\alpha]_{D}^{22}=+2.35$ (c 0.85, THF). IR (neat, $\mathrm{cm}^{-1}$ ): 3355 (s), 2928 (s), 1461 (m), 1019 (m); ${ }^{1} \mathrm{H}$ NMR (400 MHz, $\left.\mathrm{CDCl}_{3}\right)$ ठ 3.57-3.69 (m, 2H), $3.48(\mathrm{dd}, \mathrm{J}=9.2 \mathrm{~Hz}, 2.4 \mathrm{~Hz}, 1 \mathrm{H})$, 1.25-1.76 (m, 16H), $0.87(\mathrm{~s}, 3 \mathrm{H}) ;{ }^{13} \mathrm{C}$ NMR $\left(\mathrm{CDCl}_{3}, 100 \mathrm{MHz}\right) \delta \mathrm{u}: 63.9,39.9,36.7,36.1$, 33.5, 28.7, 27.1, 24.7, 22.1; d: 78.6, 20.5; HRMS calcd for $\mathrm{C}_{11} \mathrm{H}_{21} \mathrm{O}$ (M-OH) 169.1592, found 169.1590 .

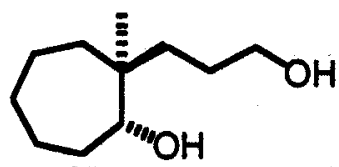

25

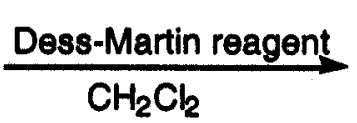
$\mathrm{CH}_{2} \mathrm{Cl}_{2}$

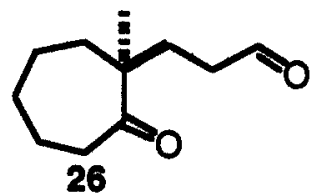

\section{(-)-Aldehyde 26}

To a stirred suspension of Dess-Martin reagent $(349 \mathrm{mg}, 0.82 \mathrm{mmol})$ in $\mathrm{CH}_{2} \mathrm{Cl}_{2}(2 \mathrm{~mL})$ was added a solution of diol $25(51 \mathrm{mg})$ in $\mathrm{CH}_{2} \mathrm{Cl}_{2}(2 \mathrm{~mL})$ slowly over $5 \mathrm{~min}$. The mixture was stirred at room temperature for $30 \mathrm{~min}$. Silica gel $(2.0 \mathrm{~g})$ was added and the mixture was concentrated. The residual solid was chromatographed to give $46 \mathrm{mg}$ of aldehyde 26 (92\% yield) as a colorless oil, TLC $R_{f}=0.54$ (PE:MTBE, $\left.1: 1\right),[\alpha]_{\mathrm{D}}^{22}=-29.3$ (c 1.1, THF). IR (neat, $\mathrm{cm}^{-1}$ ): 2928 (s), 2857 (m), 1723 (s), 1698 (s), 1458 (m); ${ }^{1} \mathrm{H}$ NMR 
$\left(400 \mathrm{MHz}, \mathrm{CDCl}_{3}\right) \delta 9.75(\mathrm{~s}, 1 \mathrm{H}), 2.37-2.69(\mathrm{~m}, 4 \mathrm{H}), 1.28-1.92(\mathrm{~m}, 10 \mathrm{H}), 1.04(\mathrm{~s}, 3 \mathrm{H}) ;$

${ }^{13} \mathrm{C}$ NMR $\left(\mathrm{CDCl}_{3}, 100 \mathrm{MHz}\right) \delta \mathrm{u}: 217.3,50.1,40.4,39.2,38.1,31.2,30.7,26.7,24.6 ; \mathrm{d}:$

201.9, 21.5; HRMS calcd for $\mathrm{C}_{11} \mathrm{H}_{19} \mathrm{O}_{2}(\mathrm{M}+\mathrm{H})$ 183.1385, found 183.1379 .

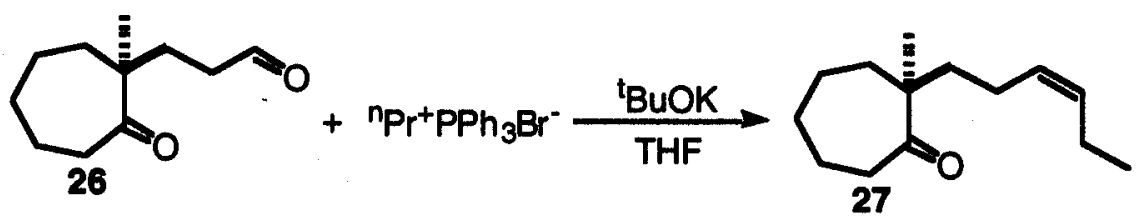

\section{(-)-Ketone 27}

To a stirred solution of ${ }^{\mathrm{B}} \mathrm{BuOK}(56.8 \mathrm{mg}, 0.506 \mathrm{mmol})$ in dry THF ( $\left.2 \mathrm{~mL}\right)$ was added n-propyltriphenylphosphium bromide ( $205 \mathrm{mg}, 0.531 \mathrm{mmol}$ ) in two portions over $2 \mathrm{~min}$ at $0^{\circ} \mathrm{C}$. The resulting orange solution was stirred at $0^{\circ} \mathrm{C}$ for $1 \mathrm{~h}$, then room temperature for $30 \mathrm{~min}$. The solution was cooled to $0{ }^{\circ} \mathrm{C}$ again, and aldehyde $26(46 \mathrm{mg}, 0.253 \mathrm{mmol}$ ) in dry THF ( $2 \mathrm{ml})$ was added slowly. The resulting solution was stirred at room temperature overnight. The reaction mixture was partitioned between saturated aqueous $\mathrm{NH}_{4} \mathrm{Cl}$ and $\mathrm{CH}_{2} \mathrm{Cl}_{2}$. The combined organic extract was dried over $\mathrm{Na}_{2} \mathrm{SO}_{4}$ and concentrated in vacuo. The residue was chromatographed to give $45 \mathrm{mg}$ of alkene 27 (86\% yield) as a colorless oil, TLC $R_{f}=0.74$ (PE:MTBE, 4:1), $[\alpha]_{\mathrm{D}}^{22}=-35.2$ (c 0.87, THF). IR (neat, $\mathrm{cm}^{-1}$ ): 2930 (s), 1701 (s), 1458 (m); ${ }^{1} \mathrm{H}$ NMR (400 MHz, $\mathrm{CDCl}_{3}$ ) $\delta 5.35$ (m, 1H), $5.27(\mathrm{~m}, 1 \mathrm{H}), 2.68(\mathrm{~m}, 1 \mathrm{H}), 2.35(\mathrm{~m}, 1 \mathrm{H}), 1.24-2.02(\mathrm{~m}, 14 \mathrm{H}), 1.06(\mathrm{~s} .3 \mathrm{H}), 0.94$ $(\mathrm{t}, \mathrm{J}=7.2 \mathrm{~Hz}, 3 \mathrm{H}) ;{ }^{13} \mathrm{C} \mathrm{NMR}\left(\mathrm{CDCl}_{3}, 100 \mathrm{MHz}\right) \delta \mathrm{u}: 217.9,51.1,40.4,40.3,38.0,30.9$, 26.9, 24.6, 22.1, 20.6; d: 132.2, 128.7, 21.5, 14.5; HRMS calcd for $\mathrm{C}_{14} \mathrm{H}_{25} \mathrm{O}(\mathrm{M}+\mathrm{H})$ 209.1905, found 209.1903. 


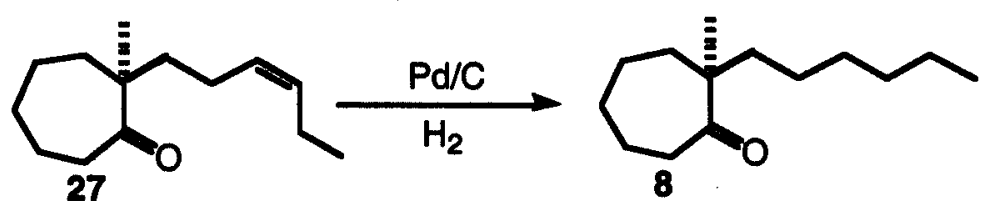

\section{(-)-Ketone $8^{6}$}

To a solution of alkene $27(45 \mathrm{mg}, 0.216 \mathrm{mmol})$ in absolute ethanol $(4 \mathrm{~mL})$ was added $10 \% \mathrm{Pd} / \mathrm{C}(10 \mathrm{mg})$. The mixture was stirred under $\mathrm{H}_{2}$ atmosphere $(1 \mathrm{~atm})$ for $5 \mathrm{~h}$. Silica gel $(500 \mathrm{mg})$ was added and the mixture was concentrated. The residual solid was chromatographed to give $44 \mathrm{mg}$ of ketone 8 (92\% yield) as a colorless oil, $[\alpha]^{22}{ }_{D}=-36.3$ (c 0.9, THF). IR (neat, $\left.\mathrm{cm}^{-1}\right): 2929$ (s), 2856 (m), 1701 (s), 1465 (m); ${ }^{1} \mathrm{H}$ NMR (400 $\left.\mathrm{MHz}, \mathrm{CDCl}_{3}\right) \delta 2.67(\mathrm{~m}, 1 \mathrm{H}), 2.33(\mathrm{~m}, 1 \mathrm{H}), 1.18-1.83(\mathrm{~m}, 18 \mathrm{H}), 1.03(\mathrm{~s}, 3 \mathrm{H}), 0.87(\mathrm{t}$, $\mathrm{J}=6.8 \mathrm{~Hz}, 3 \mathrm{H}) ;{ }^{13} \mathrm{C} \mathrm{NMR}\left(\mathrm{CDCl}_{3}, 100 \mathrm{MHz}\right) \delta \mathrm{u}: 218.4,51.1,40.5,40.4,38.0,31.9,30.9$, $30.1,26.9,24.6,24.1,22.8 ;$ d: $21.7,14.3$.

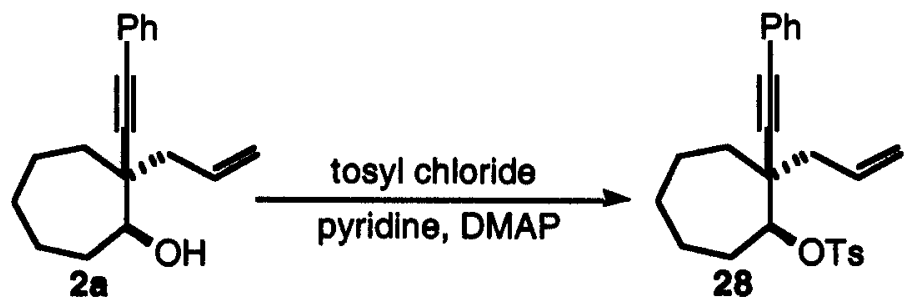

\section{Tosylate 28}

To a stirred solution of secondary alcohol $2 \mathrm{a}(9.50 \mathrm{~g}, 37.4 \mathrm{mmol})$ in dry pyridine (80 $\mathrm{mL}$ ) was added DMAP $(0.457 \mathrm{~g}, 3.74 \mathrm{mmol})$ and tosyl chloride $(14.3 \mathrm{~g}, 74.8 \mathrm{mmol})$. The resulting solution was stirred under $\mathrm{N}_{2}$ at room temperature for $36 \mathrm{~h}$. The reaction mixture was partitioned between ether and $5 \% \mathrm{HCl}$. The ether layer was dried over 
$\mathrm{Na}_{2} \mathrm{SO}_{4}$ and concentrated in vacuo. The residue was chromatographed to give $13.9 \mathrm{~g}$ of tosylate 28 (91\% yield) as a colorless oil, TLC $R_{f}=0.67$ (PE:MTBE, 4:1). IR (neat, $\mathrm{cm}^{-}$

1): 2938 (s), 1640 (w), 1449 (m), 1363 (s), 1185 (s); ${ }^{1} \mathrm{H}$ NMR (400 MHz, $\left.\mathrm{CDCl}_{3}\right) \delta 7.80-$ $7.84(\mathrm{~m}, 2 \mathrm{H}), 7.36-7.40(\mathrm{~m}, 2 \mathrm{H}), 7.25-7.29(\mathrm{~m}, 5 \mathrm{H}), 5.95(\mathrm{~m}, 1 \mathrm{H}), 5.08-5.16(\mathrm{~m}, 2 \mathrm{H})$, $4.48(\mathrm{dd}, \mathrm{J}=10.0 \mathrm{~Hz}, 2.8 \mathrm{~Hz}, 1 \mathrm{H}), 2.61(\mathrm{dd}, \mathrm{J}=14.0 \mathrm{~Hz}, 6.0 \mathrm{~Hz}, 1 \mathrm{H}), 2.39$ (s, 3H), 2.13 (dd, J=14.0 Hz, $9.2 \mathrm{~Hz}, 1 \mathrm{H}), 1.40-1.97(\mathrm{~m}, 10 \mathrm{H}) ;{ }^{13} \mathrm{C} \mathrm{NMR}\left(\mathrm{CDCl}_{3}, 100 \mathrm{MHz}\right) \delta \mathrm{u}$ : $144.5,134.8,123.5,118.7,90.4,85.2,44.6,43.6,34.2,31.5,26.1,22.0,21.9 ;$ d: 134.0 , $131.7,129.7,128.2,127.9,127.6,88.3,21.6 ; \mathrm{HRMS}$ calcd for $\mathrm{C}_{25} \mathrm{H}_{28} \mathrm{O}_{3} \mathrm{NaS}(\mathrm{M}+\mathrm{Na})$ 431.1657, found 431.1656 .

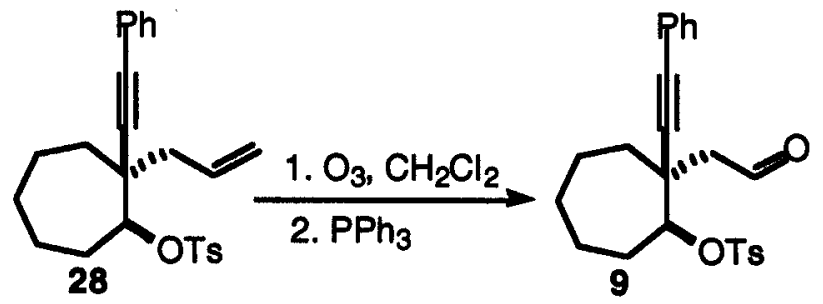

\section{Aldehyde 9}

A solution of tosylate $28(6.0 \mathrm{~g}, 14.7 \mathrm{mmol})$ in $\mathrm{CH}_{2} \mathrm{Cl}_{2}(200 \mathrm{~mL})$ was cooled to $-78^{\circ} \mathrm{C}$. After purging with $\mathrm{O}_{2}$ for $3 \mathrm{~min}, \mathrm{O}_{3}$ then was bubbled through the solution until the pale blue color persisted. The reaction mixture was purged with $\mathrm{N}_{2}$ for $5 \mathrm{~min}$, then $\mathrm{PPh}_{3}(5.01$ $\mathrm{g}, 19.1 \mathrm{mmol}$ ) was added. The resuling solution was first stirred at $-78^{\circ} \mathrm{C}$ for $1 \mathrm{~h}$, then room temperature for $1 \mathrm{~h}$. Silica gel $(20.0 \mathrm{~g})$ was added and the mixture was concentrated. The residual solid was chromatographed to give $5.48 \mathrm{~g}$ of aldehyde 9 ( $91 \%$ yield) as a colorless oil, TLC $R_{f}=0.29$ (PE:MTBE, 4:1). IR (neat, $\mathrm{cm}^{-1}$ ): 2935 (s), 2864 (m), 1722 (s), 1598 (m), 1491 (m), 1443 (m), 1362 (s), 1175 (s); ${ }^{1} \mathrm{H}$ NMR (400 MHz, 
$\left.\mathrm{CDCl}_{3}\right) \delta 9.82(\mathrm{dd}, \mathrm{J}=3.6 \mathrm{~Hz}, 1.6 \mathrm{~Hz}, 1 \mathrm{H}), 7.80(\mathrm{~d}, \mathrm{~J}=6.4 \mathrm{~Hz}, 2 \mathrm{H}), 7.28-7.40(\mathrm{~m}, 7 \mathrm{H})$, 4.48 (dd, J=10.4 Hz, 2.8 Hz, 1H), 2.79 (dd, J=16 Hz, 1.6 Hz, 1H), 2.42 (s, 3H), 2.38 (dd, $\mathrm{J}=16 \mathrm{~Hz}, 3.6 \mathrm{~Hz}, 1 \mathrm{H}), 1.45-2.17(\mathrm{~m}, 10 \mathrm{H}) ;{ }^{13} \mathrm{C} \mathrm{NMR}\left(\mathrm{CDCl}_{3}, 100 \mathrm{MHz}\right) \delta \mathrm{u}: 145.0$, 134.2, 122.8, 88.1, 87.0, 52.1, 42.8, 36.2, 31.3, 25.7, 22.2, 21.8; d: 201.6, 131.7, 129.9, $128.4,128.3,127.8,87.6,21.7 ; \mathrm{HRMS}$ calcd for $\mathrm{C}_{24} \mathrm{H}_{26} \mathrm{O}_{4} \mathrm{~S}\left(\mathrm{M}^{+}\right) 410.1552$, found 410.1569.

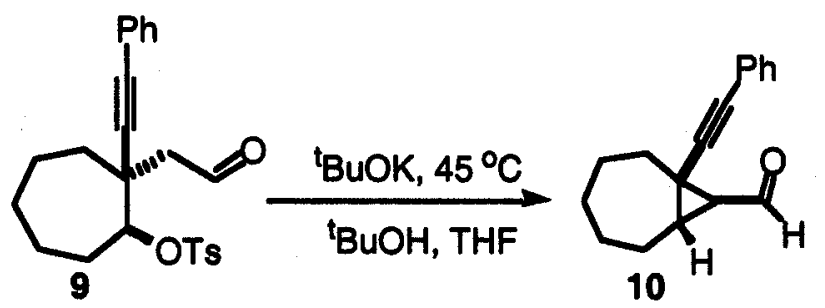

\section{Aldehyde 10}

A solution of aldehyde $9(4.1 \mathrm{~g}, 10 \mathrm{mmol})$ in $100 \mathrm{~mL}$ of a $1: 1$ mixture of dry THF and tert-butanol was heated to $45^{\circ} \mathrm{C}$ under $\mathrm{N}_{2}$, then a solution of 'BuOK $(1.46 \mathrm{~g}, 13 \mathrm{mmol})$ in $60 \mathrm{~mL}$ of a 1:1 mixture of dry THF and tert-butanol was added slowly over $10 \mathrm{~min}$. The resulting solution was stired at this temperature for $1.5 \mathrm{~h}$. The reaction mixture was partitioned between water and ether. The combined organic extract was dried over $\mathrm{Na}_{2} \mathrm{SO}_{4}$ and concentrated in vacuo. The residue was chromatographed to give $2.1 \mathrm{~g}$ of aldehyde 10 (88\% yield) as a colorless oil, TLC $R_{f}=0.68$ (PE:MTBE, 4:1). IR (neat, $\mathrm{cm}^{-}$ 1): 2925 (s), 2852 (m), 2221 (w), 1709 (s); ${ }^{1} \mathrm{H}$ NMR (400 MHz, $\mathrm{CDCl}_{3}$ ) $\delta 9.21$ (d, J=6.8 $\mathrm{Hz}, 1 \mathrm{H}), 7.35-7.40(\mathrm{~m}, 2 \mathrm{H}), 7.25-7.29(\mathrm{~m}, 3 \mathrm{H}), 2.41(\mathrm{dd}, \mathrm{J}=14.4 \mathrm{~Hz}, 6.8 \mathrm{~Hz}, 1 \mathrm{H}), 2.29$ $(\mathrm{m}, 1 \mathrm{H}), 2.08(\mathrm{~m}, 1 \mathrm{H}), 1.91(\mathrm{~m}, 1 \mathrm{H}), 1.08-1.84(\mathrm{~m}, 8 \mathrm{H}) ;{ }^{13} \mathrm{C} \mathrm{NMR}\left(\mathrm{CDCl}_{3}, 100 \mathrm{MHz}\right) \delta$ 
u: $123.1,91.3,80.2,35.7,31.9,29.8,28.1,27.3,26.6$; d: 200.6, 131.7, 128.3, 128.1, 46.5, 34.4; HRMS calcd for $\mathrm{C}_{17} \mathrm{H}_{18} \mathrm{O}\left(\mathrm{M}^{+}\right)$238.1358, found 238.1352 .

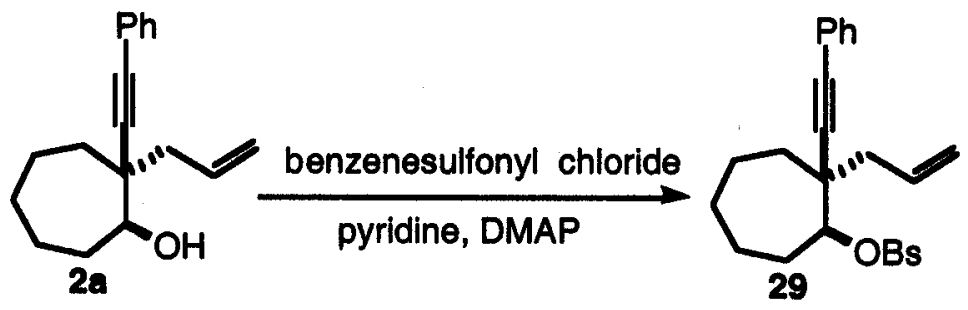

\section{Benzenesulfonate 29}

To a stirred solution of alcohol $2 \mathrm{a}(7.60 \mathrm{~g}, 29.9 \mathrm{mmol})$ in dry pyridine $(40 \mathrm{~mL})$ was added DMAP (0.365 g, $2.99 \mathrm{mmol})$ and benzenesulfonyl chloride $(10.6 \mathrm{~g}, 59.8 \mathrm{mmol})$. The resulting solution was stirred under $\mathrm{N}_{2}$ at room temperature for $40 \mathrm{~h}$. The reaction mixture was partitioned between ether and $10 \% \mathrm{HCl}$. The ether layer was dried over $\mathrm{Na}_{2} \mathrm{SO}_{4}$ and concentrated in vacuo. The residue was chromatographed to give $10.8 \mathrm{~g}$ of benzenesulfonate 29 (92\% yield) as a colorless oil, TLC $R_{f}=0.72$ (PE:MTBE, 4:1). IR (neat, $\mathrm{cm}^{-1}$ ): 2937 (s), 1640 (w), 1490 (w), 1448 (m), 1363 (s), 1185 (s); ${ }^{1}$ H NMR (400 $\left.\mathrm{MHz}, \mathrm{CDCl}_{3}\right)$ ठ 7.91-7.94 (m, 2H), 7.23-7.60 (m, 8H), $5.91(\mathrm{~m}, 1 \mathrm{H})$, 5.05-5.13 (m, 2H), 4.47 (dd, J=10.0 Hz, $2.8 \mathrm{~Hz}, 1 \mathrm{H}), 1.46-2.59(\mathrm{~m}, 12 \mathrm{H}) ;{ }^{13} \mathrm{C} N M R$ $\left(\mathrm{CDCl}_{3}, 100 \mathrm{MHz}\right) \delta \mathrm{u}: 138.3,123.9,119.2,90.7,85.7,45.1,44.1,34.6,32.1,26.6,22.5$, 22.4; d: 134.4, 134.0, 132.2, 129.6, 128.6, 128.4, 128.0, 89.1; HRMS calcd for $\mathrm{C}_{24} \mathrm{H}_{26} \mathrm{O}_{3} \mathrm{~S}\left(\mathrm{M}^{+}\right)$394.1603, found 394.1609. 


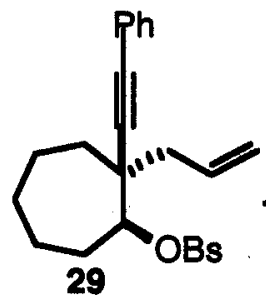

\section{1. $\mathrm{O}_{3}, \mathrm{CH}_{2} \mathrm{Cl}_{2}, \mathrm{MeOH} ; \mathrm{NaBH}_{4}$ \\ 2. Methanesulfonyl chloride, $\mathrm{CH}_{2} \mathrm{Cl}_{2}, \mathrm{Et}_{3} \mathrm{~N}$}

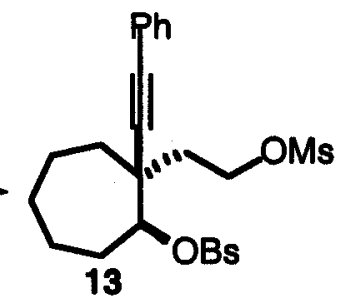

\section{Methanesulfonate 13}

A solution of benzenesulfonate $29(7.88 \mathrm{~g}, 20.0 \mathrm{mmol})$ in HPLC grade methanol (50 $\mathrm{mL}$ ) and $\mathrm{CH}_{2} \mathrm{Cl}_{2}(50 \mathrm{~mL})$ was cooled to $-78^{\circ} \mathrm{C}$. After purging with $\mathrm{O}_{2}$ for $2 \mathrm{~min}, \mathrm{O}_{3}$ was bubbled through the solution until the pale blue color persisted. The reaction was purged with $\mathrm{N}_{2}$ for $5 \mathrm{~min}$, then $\mathrm{NaBH}_{4}(1.50 \mathrm{~g}, 40.0 \mathrm{mmol})$ was added. After stirring at $-78^{\circ} \mathrm{C}$ for $10 \mathrm{~min}$, the reaction mixture was cooled with an ice-water bath. After an additional 30 min, $\mathrm{NaBH}_{4}(750 \mathrm{mg}, 20.0 \mathrm{mmol})$ was added followed by stirring for another $30 \mathrm{~min}$. The latter procedure was repeated once more. The reaction mixture was partitioned between water and $\mathrm{CH}_{2} \mathrm{Cl}_{2}$. The combined organic extract was dried $\left(\mathrm{Na}_{2} \mathrm{SO}_{4}\right)$ and concentrated, and the residual clear oil was dissolved in $150 \mathrm{~mL}$ of anhydrous $\mathrm{CH}_{2} \mathrm{Cl}_{2}$. To this solution triethylamine $(6.07 \mathrm{~g}, 60.0 \mathrm{mmol})$ was added, then methanesulfonyl chloride $(3.44 \mathrm{~g}, 30.0 \mathrm{mmol})$ was added slowly at $0{ }^{\circ} \mathrm{C}$. After stirring under $\mathrm{N}_{2}$ at ambient temperature for $1 \mathrm{~h}$, the reaction mixture was partitioned between saturated aqueous water and $\mathrm{CH}_{2} \mathrm{Cl}_{2}$. The combined organic extract was dried $\left(\mathrm{Na}_{2} \mathrm{SO}_{4}\right)$ and concentrated. The crude product was chromatographed to give $7.91 \mathrm{~g}$ of methanesulfonate 13 (83\% yield) as a clear oil, TLC $R_{f}=0.5$ (PE:MTBE, 1:2). IR (neat, $\mathrm{cm}^{-1}$ ): $2939(\mathrm{~s}), 1448(\mathrm{~m})$, 1357 (s), 1175 (s); ${ }^{1} \mathrm{H}$ NMR (400 MHz, $\mathrm{CDCl}_{3}$ ) $\delta$ 7.93-7.95 (m, 2H), 7.28-7.66 (m, 8H), $4.46-4.50(\mathrm{~m}, 3 \mathrm{H}), 3.00(\mathrm{~s}, 3 \mathrm{H}), 1.42-2.28(\mathrm{~m}, 12 \mathrm{H}) ;{ }^{13} \mathrm{C} \mathrm{NMR}\left(\mathrm{CDCl}_{3}, 100 \mathrm{MHz}\right) \delta \mathrm{u}:$ 
$137.6,122.9,88.6,86.3,67.6,44.1,37.9,35.7,31.3,26.1,22.1$; d: $133.9,131.9,129.4$,

128.4, 127.7, 88.5, 37.6; HRMS calcd for $\mathrm{C}_{24} \mathrm{H}_{28} \mathrm{O}_{6} \mathrm{NaS}(\mathrm{M}+\mathrm{Na}) 499.1225$, found 499.1242 .
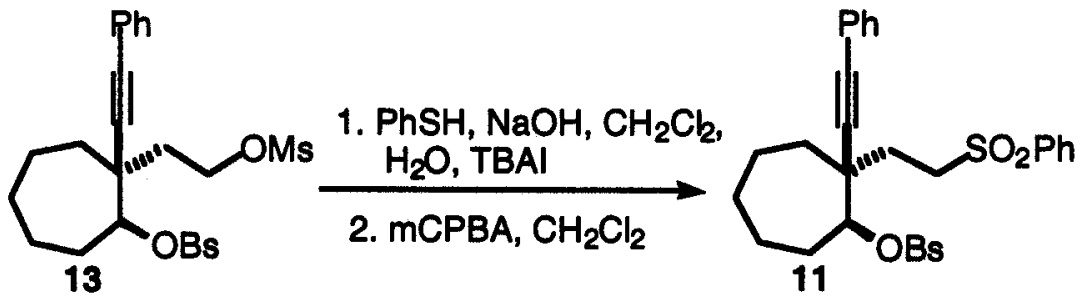

\section{Sulfone 11}

To a mixture of methanesulfonate $13(160 \mathrm{mg}, 0.338 \mathrm{mmol})$ in benzene $(2 \mathrm{~mL})$ and water ( $2 \mathrm{~mL}$ ) was added benzenethiol (112 $\mathrm{mg}, 1.01 \mathrm{mmol}), \mathrm{NaOH}$ (135 mg, $3.38 \mathrm{mmol})$ and tetrabutylammonium bromide $(11.0 \mathrm{mg}, 0.0338 \mathrm{mmol})$. The mixture was stirred vigorously under $\mathrm{N}_{2}$ at room temperature for $22 \mathrm{~h}$. The reaction mixture was partitioned between water and $\mathrm{CH}_{2} \mathrm{Cl}_{2}$. The combined $\mathrm{CH}_{2} \mathrm{Cl}_{2}$ extract was dried $\left(\mathrm{Na}_{2} \mathrm{SO}_{4}\right)$, and mCPBA (70\%, $332 \mathrm{mg}, 1.35 \mathrm{mmol})$ was added to this solution. After $10 \mathrm{~min}$ at room temperature the reaction was partitioned between $\mathrm{CH}_{2} \mathrm{Cl}_{2}$ and, sequentially, saturated aqueous $\mathrm{Na}_{2} \mathrm{~S}_{2} \mathrm{O}_{3}$, saturated aqueous $\mathrm{Na}_{2} \mathrm{CO}_{3}$ and brine. The combined organic extract was dried $\left(\mathrm{Na}_{2} \mathrm{SO}_{4}\right)$ and concentrated. The crude product was chromatographed to give $150 \mathrm{mg}$ of sulfone 11 (85\% yield) as a colorless oil, TLC $R_{f}=0.43$ (PE:MTBE, 1:1). IR (neat, $\left.\mathrm{cm}^{-1}\right): 2937$ (s), 1447 (s), 1361 (m), 1306 (s), $1186(\mathrm{~s}), 1145$ (s), 1087 (m); ${ }^{1} \mathrm{H}$ NMR (400 MHz, $\left.\mathrm{CDCl}_{3}\right)$ 反 7.87-7.91 (m, 4H), 7.50-7.66 (m, 6H), 7.24-7.31 (m, 5H), 4.40 (dd, J=11.6 Hz, $2.4 \mathrm{~Hz}, 1 \mathrm{H}), 3.28-3.41(\mathrm{~m}, 2 \mathrm{H}), 1.35-2.20(\mathrm{~m}, 12 \mathrm{H}) ;{ }^{13} \mathrm{C}$ NMR $\left(\mathrm{CDCl}_{3}, 100 \mathrm{MHz}\right) \delta \mathrm{u}: 138.9,137.3,122.8,88.3,86.5,52.8,44.6,35.0,32.0,31.7,26.0$, 
22.1, 22.0; d: 133.9, 133.8, 131.8, 129.4, 129.3, 128.4, 128.3, 128.2, 127.7, 87.8; HRMS calcd for $\mathrm{C}_{29} \mathrm{H}_{30} \mathrm{O}_{3} \mathrm{NaS}_{2}(\mathrm{M}+\mathrm{Na}) 545.1432$, found 545.1454.

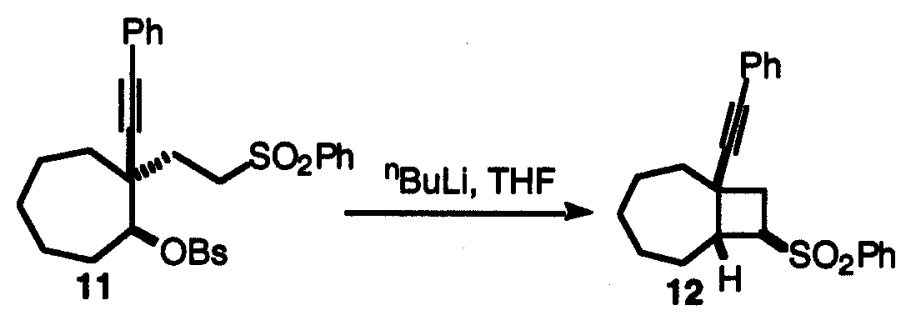

\section{Sulfone 12}

To a stirred solution of sulfone $11(63.0 \mathrm{mg}, 0.121 \mathrm{mmol})$ in dry THF $(1 \mathrm{~mL})$ was added ${ }^{\mathrm{n}} \mathrm{BuLi}\left(71 \mu \mathrm{L}, 1.80 \mathrm{M}\right.$ in hexane) under $\mathrm{N}_{2}$ at $-78^{\circ} \mathrm{C}$. The reaction mixture was stirred at ambient temperature for $1 \mathrm{~h}$, then was partitioned between saturated aqueous $\mathrm{NH}_{4} \mathrm{Cl}$ and ehter. The combined organic extract was dried $\left(\mathrm{Na}_{2} \mathrm{SO}_{4}\right)$ and concentrated. The crude product was chromatographed to give $36 \mathrm{mg}$ of sulfone 12 (82\% yield) as a colorless oil, TLC $R_{f}=0.58$ (PE:MTBE, 1:1). IR (neat, $\mathrm{cm}^{-1}$ ): 2925 (s), $2854(\mathrm{~m}), 1446$ (m), 1301 (s), 1149 (s); ${ }^{1} \mathrm{H}$ NMR (400 MHz, $\left.\mathrm{CDCl}_{3}\right)$ 8 7.89-7.93 (m, 2H), 7.27-7.66 (m, $8 \mathrm{H}), 3.40(\mathrm{~m}, 1 \mathrm{H}), 3.19(\mathrm{~m}, 1 \mathrm{H}), 2.84(\mathrm{dd}, \mathrm{J}=12 \mathrm{~Hz}, 9.2 \mathrm{~Hz}, 1 \mathrm{H}), 2.15$ (dd, J=12 Hz, 8.8 $\mathrm{Hz}, 1 \mathrm{H}), 1.22-2.18(\mathrm{~m}, 10 \mathrm{H}) ;{ }^{13} \mathrm{C} \mathrm{NMR}\left(\mathrm{CDCl}_{3}, 100 \mathrm{MHz}\right) \delta \mathrm{u}: 138.9,123.9,95.4,84.5$, $36.8,36.4,33.7,33.5,32.2,27.0,26.2 ; \mathrm{d}: 133.8,131.8,129.5,128.6,128.4,128.0,60.0$, 47.2; HRMS calcd for $\mathrm{C}_{23} \mathrm{H}_{24} \mathrm{O}_{2} \mathrm{~S}\left(\mathrm{M}^{+}\right)$364.1497, found 364.1507. 

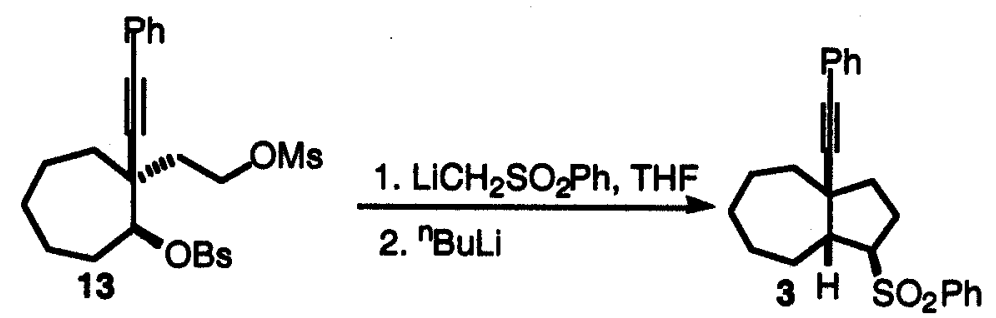

\section{Sulfone 3}

To a stirred solution of methyl phenyl sulfone $(145 \mathrm{mg}, 0.930 \mathrm{mmol})$ in dry THF (1 $\mathrm{mL}$ ) was added ${ }^{\mathrm{n}} \mathrm{BuLi}\left(0.37 \mathrm{~mL}, 2.5 \mathrm{M}\right.$ in hexane) at $-78^{\circ} \mathrm{C}$ under $\mathrm{N}_{2}$. After stirring at this temperature for $5 \mathrm{~min}$, a solution of methanesulfonate $13(400 \mathrm{mg}, 0.840 \mathrm{mmol})$ in dry THF ( $2 \mathrm{~mL}$ ) was added slowly. The resulting mixture then was stirred at ambient temperature for $19 \mathrm{~h}$. The reaction was cooled to $-78^{\circ} \mathrm{C}$, and ${ }^{\mathrm{n}} \mathrm{BuLi}(0.37 \mathrm{~mL}, 2.5 \mathrm{M}$ in hexane) was added slowly. After stirring at ambient temperature for $2 \mathrm{~h}$, the reaction mixture was partitioned between saturated aqueous $\mathrm{NH}_{4} \mathrm{Cl}$ and ether. The combined organic extract was dried $\left(\mathrm{Na}_{2} \mathrm{SO}_{4}\right)$ and concentrated. The crude product $\mathrm{w}$ as chromatographed to give $230 \mathrm{mg}$ of sulfone 3 ( $72 \%$ yield) as a white solid: $\mathrm{mp}=121-122$ ${ }^{\circ} \mathrm{C}$, TLC $R_{f}=0.85$ (PE:MTBE, 1:2). IR (film, $\mathrm{cm}^{-1}$ ): 2926 (s), 2854 (m), 1490 (s), 1446 (m), 1301 (s), 1147 (s), $1086(\mathrm{~m}) ;{ }^{1} \mathrm{H}$ NMR $\left(400 \mathrm{MHz}, \mathrm{CDCl}_{3}\right) \delta$ 7.90-7.95 (m, 2H), 7.47-7.53 (m, 3H), 7.24-7.34 (m, 5H), $3.13(\mathrm{t} \mathrm{d}, \mathrm{J}=7.2 \mathrm{~Hz}, 10.8 \mathrm{~Hz}, 1 \mathrm{H}), 2.78(\mathrm{~m}, 1 \mathrm{H})$, $2.27(\mathrm{~m}, 1 \mathrm{H}), 2.02(\mathrm{~m}, 1 \mathrm{H}), 1.23-1.97(\mathrm{~m}, 12 \mathrm{H}) ;{ }^{13} \mathrm{C} \mathrm{NMR}\left(\mathrm{CDCl}_{3}, 100 \mathrm{MHz}\right) \delta \mathrm{u}: 138.1$, $123.9,97.1,82.5,47.5,41.2,37.8,33.6,30.8,29.2,27.9,25.6$; d: $133.7,131,8,129.2$, 129.1, 128.2, 127.7, 72.1, 52.1; HRMS calcd for $\mathrm{C}_{24} \mathrm{H}_{26} \mathrm{O}_{2} \mathrm{~S}\left(\mathrm{M}^{+}\right)$378.1654, found 378.1658 . 


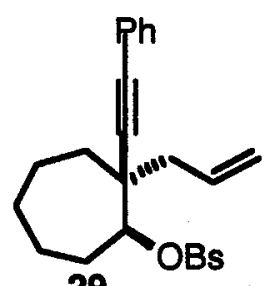

29

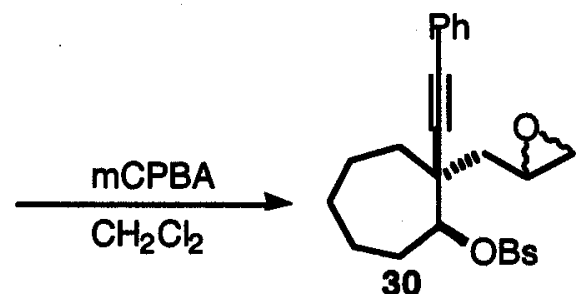

30

\section{Epoxide 30}

To a stirred solution of benzenesulfonate $29(9.1 \mathrm{~g}, 23.1 \mathrm{mmol})$ in $\mathrm{CH}_{2} \mathrm{Cl}_{2}(100 \mathrm{~mL})$ was added mCPBA $(70 \%, 6.82 \mathrm{~g}, 27.7 \mathrm{mmol})$. The resulting mixture was stirred at ambient temperature for $40 \mathrm{~h}$. The reaction mixture then was partitioned between $2 \mathrm{~N}$ aqueous $\mathrm{NaOH}$ and ether. The combined organic extract was dried $\left(\mathrm{Na}_{2} \mathrm{SO}_{4}\right)$ and concentrated. The crude product was chromatographed to give $9.0 \mathrm{~g}$ of epoxide 30 (95\% yield) as a colorless oil, TLC $R_{f}=0.53$ (PE:MTBE, 1:1). IR (neat, $\mathrm{cm}^{-1}$ ): 2935 (s), 1448 (m), $1362(\mathrm{~s}), 1185(\mathrm{~s}) ;{ }^{1} \mathrm{H}$ NMR (400 MHz, $\left.\mathrm{CDCl}_{3}\right) \delta$ major isomer: 7.27-7.60 (m, 10H), $4.60(\mathrm{dd}, \mathrm{J}=10.0 \mathrm{~Hz}, 2.4 \mathrm{~Hz}, 1 \mathrm{H}), 3.16(\mathrm{~m}, 1 \mathrm{H}), 2.77(\mathrm{t}, \mathrm{J}=4.6 \mathrm{~Hz}, 1 \mathrm{H}), 2.46$ (dd, J= 4.6 $\mathrm{Hz}, 2.4 \mathrm{~Hz}, 1 \mathrm{H}), 1.36-2.30(\mathrm{~m}, 12 \mathrm{H})$; minor isomer: 7.27-7.60 (m, 10H), $4.40(\mathrm{dd}, \mathrm{J}=$ $10.4 \mathrm{~Hz}, 2.4 \mathrm{~Hz}, 1 \mathrm{H}), 3.25(\mathrm{~m}, 1 \mathrm{H}), 2.74(\mathrm{t}, \mathrm{J}=4.4 \mathrm{~Hz}, 1 \mathrm{H}), 2.40$ (dd, J=4.4 Hz, $2.4 \mathrm{~Hz}$ $1 \mathrm{H}), 1.36-2.30(\mathrm{~m}, 12 \mathrm{H}) ;{ }^{13} \mathrm{C} \mathrm{NMR}\left(\mathrm{CDCl}_{3}, 100 \mathrm{MHz}\right) \delta$ (two isomers are reported together): u: $137.7,137.6,123.3,89.0,88.5,85.9,85.4,47.4,45.8,45.1,44.3,42.2,35.5$, $34.8,31.5,31.2,26.1,22.2,22.1,22.0,21.9 ;$ d: $133.7,131.8,129.3,128.3,128.2,128.1$, 128.0, 127.7, 89.3, 87.9, 49.8, 49.1; HRMS calcd for $\mathrm{C}_{24} \mathrm{H}_{26} \mathrm{O}_{4} \mathrm{~S}\left(\mathrm{M}^{+}\right) 410.1552$, found 410.1547 . 


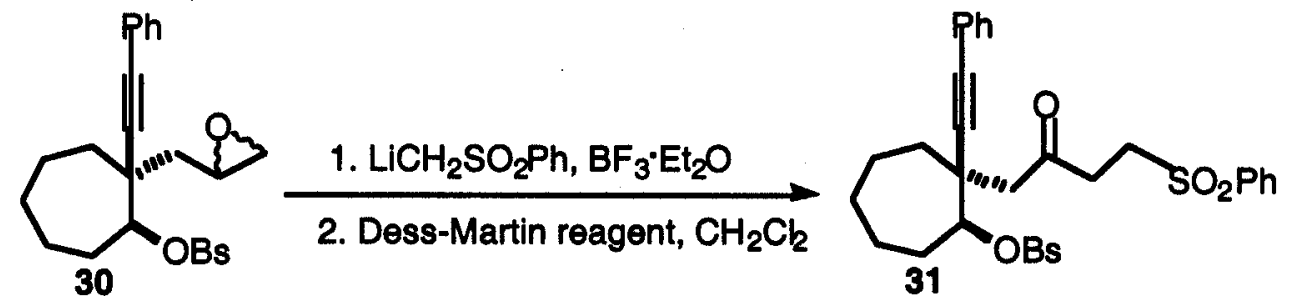

\section{Ketone 31}

To a stirred solution of methyl phenyl sulfone $(1.22 \mathrm{~g}, 7.79 \mathrm{mmol})$ in $10 \mathrm{~mL}$ of dry THF was added ${ }^{\mathrm{B}} \mathrm{BuLi}(2.25 \mathrm{M}, 3.46 \mathrm{~mL})$ dropwise at $-78^{\circ} \mathrm{C}$ under $\mathrm{N}_{2}$. After stirring for $5 \mathrm{~min}$, a solution of $\mathrm{BF}_{3} \cdot \mathrm{Et}_{2} \mathrm{O}(1.01 \mathrm{~g}, 7.14 \mathrm{mmol})$ in $1 \mathrm{~mL}$ of dry THF was added, followed by the addition of epoxide $30(2.66 \mathrm{~g}, 6.49 \mathrm{mmol})$ in $5 \mathrm{~mL}$ of dry THF dropwise. The cold bath was removed, and the reaction mixture was stirred at ambient temperature for $70 \mathrm{~min}$. The reaction mixture was partitioned between saturated aqueous $\mathrm{NH}_{4} \mathrm{Cl}$ and ether. The combined organic extract was dried $\left(\mathrm{Na}_{2} \mathrm{SO}_{4}\right)$ and concentrated. The crude product was chromatographed to give $2.64 \mathrm{~g}$ of secondary alcohol as a colorless oil. This alcohol was dissolved immediately in $40 \mathrm{~mL}$ of $\mathrm{CH}_{2} \mathrm{Cl}_{2}$, and to this solution Dess-Martin reagent $(2.18 \mathrm{~g}, 5.15 \mathrm{mmol})$ was added. The resulting mixture was stirred at room temperature for $30 \mathrm{~min}$. Silica gel $(8.0 \mathrm{~g})$ was added and the mixture was concentrated. The residual solid was chromatographed to give $2.47 \mathrm{~g}$ of ketone 31 (68\% yield for two steps) as a colorless oil, TLC $R_{f}=0.47$ (PE:MTBE, 1:2). IR (neat, $\mathrm{cm}^{-1}$ ): 2932 (s), 1720 (s), 1447 (s), 1359 (s), 1308 (s), 1185 (s), 1150 (s); ${ }^{1} \mathrm{H}$ NMR (400 MHz, $\left.\mathrm{CDCl}_{3}\right) \delta$ 7.86-7.89 (m, 4H), 7.51-7.65 (m, 6H), 7.28-7.39 (m, 5H), $4.74(\mathrm{dd}, \mathrm{J}=10.8 \mathrm{~Hz}$, 3.2 Hz, 1H), 3.31-3.45 (m, $2 \mathrm{H}), 2.81-3.01(\mathrm{~m}, 4 \mathrm{H}), 1.36-2.04(\mathrm{~m}, 10 \mathrm{H}) ;{ }^{13} \mathrm{C} \mathrm{NMR}$ $\left(\mathrm{CDCl}_{3}, 100 \mathrm{MHz}\right) \delta$ u: 203.7, 139.0, 137.5, 122.9, 89.1, 85.7, 50.9, 50.6, 43.8, 36.4, 
$34.0,31.5,25.5,22.0,21.6$; d: $134.0,133.9,131.8,129.5,129.4,128.4,128.1,127.8$, 127.5, 87.0; HRMS calcd for $\mathrm{C}_{31} \mathrm{H}_{32} \mathrm{O}_{6} \mathrm{NaS}_{2}(\mathrm{M}+\mathrm{Na})$ 587.1538, found 587.1539.
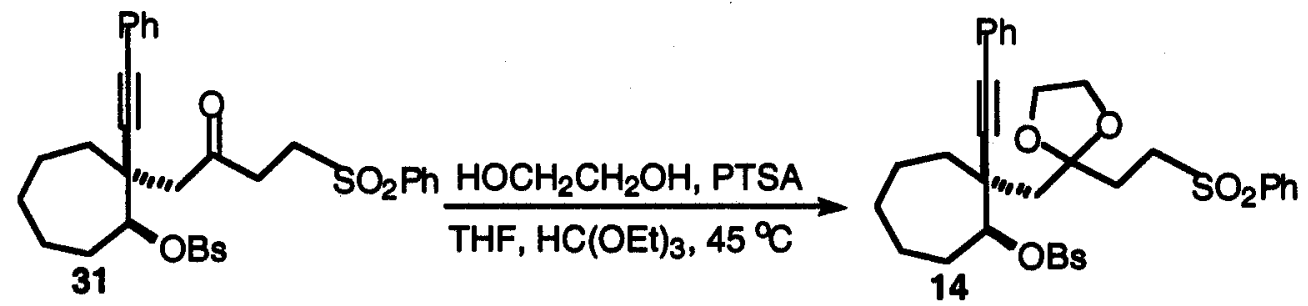

\section{Ketal 14}

A solution of ketone $31(1.50 \mathrm{~g}, 2.66 \mathrm{mmol})$, dry ethylene glycol $(1.65 \mathrm{~g}, 26.6 \mathrm{mmol})$, freshly-distilled triethylformate $(1.18 \mathrm{~g}, 7.98 \mathrm{mmol}), p$-toluenesulfonic acid monohydrate (50.6 mg, $0.266 \mathrm{mmol})$ in dry THF $(2.5 \mathrm{~mL})$ was heated at $45^{\circ} \mathrm{C}$ under $\mathrm{N}_{2}$ for $39 \mathrm{~h}$. The reaction mixture was partitioned between saturated aqueous $\mathrm{NaHCO}_{3}$ and ether. The combined organic extract was dried $\left(\mathrm{Na}_{2} \mathrm{SO}_{4}\right)$ and concentrated. The crude product was chromatographed to give $1.33 \mathrm{~g}$ of ketal 14 (82\% yield) as a colorless oil, TLC $R_{f}=0.33$ (PE:MTBE, 1:2). IR (neat, $\mathrm{cm}^{-1}$ ): 2932 (s), 1447 (s), 1362 (s), 1307 (s), 1186 (s), 1148 (s); ${ }^{1} \mathrm{H}$ NMR (400 MHz, $\mathrm{CDCl}_{3}$ ) 8 7.89-7.92 (m, 2H), 7.71-7.74 (m, 2H), 7.48-7.63 (m, 4H), 7.30-7.42 (m, 7H), $4.43(\mathrm{dd}, \mathrm{J}=10.4 \mathrm{~Hz}, 2.4 \mathrm{~Hz}, 1 \mathrm{H}), 3.74-3.90(\mathrm{~m}, 4 \mathrm{H}), 3.09-3.25$ $(\mathrm{m}, 2 \mathrm{H}), 1.38-2.36(\mathrm{~m}, 14 \mathrm{H}) ;{ }^{13} \mathrm{C} \mathrm{NMR}\left(\mathrm{CDCl}_{3}, 100 \mathrm{MHz}\right) \delta \mathrm{u}: 139.4,138.1,123.6$, $110.0,90.7,85.7,65.0,64.7,51.7,44.6,43.0,34.7,31.6,31.1,26.6,22.4,22.0 ;$ d: 133.7 , 133.6, 132.0, 129.3, 128.5, 128.2, 128.1, 127.7, 89.9; HRMS calcd for $\mathrm{C}_{33} \mathrm{H}_{36} \mathrm{O}_{7} \mathrm{NaS}_{2}$ $(\mathrm{M}+\mathrm{Na}) 631.1800$, found 631.1796 . 


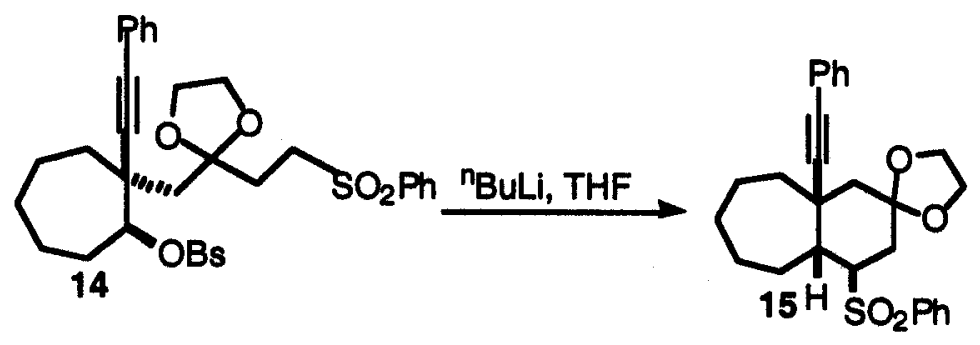

\section{Sulfone 15}

To a stirred solution of ketal $14(555 \mathrm{mg}, 0.913 \mathrm{mmol})$ in dry THF $(10 \mathrm{~mL})$ was added ${ }^{n} \mathrm{BuLi}(1.8 \mathrm{M}, 0.56 \mathrm{~mL})$ at $-78^{\circ} \mathrm{C}$ under $\mathrm{N}_{2}$. The cold bath was removed and the reaction mixture was stirred at ambient temperature for $1 \mathrm{~h}$. The reaction was partitioned between saturated aqueous $\mathrm{NH}_{4} \mathrm{Cl}$ and ether. The combined organic extract was dried $\left(\mathrm{Na}_{2} \mathrm{SO}_{4}\right)$ and concentrated. The crude product was chromatographed to give $378 \mathrm{mg}$ of sulfone 15 (92\% yield) as a colorless oil, TLC $R_{f}=0.54$ (PE:MTBE, 1:2). IR (neat, $\left.\mathrm{cm}^{-1}\right): 2929$ (s), 1490 (m), 1446 (s), 1304 (s), 1145 (s); ${ }^{1} \mathrm{H}$ NMR (400 MHz, $\mathrm{CDCl}_{3}$ ) $\delta$ 7.83-7.91 (m, 2H), 7.43-7.52 (m, 3H), 7.25 (br, 5H), 3.71-3.92 (m, 4H), $3.26(\mathrm{~m}, 1 \mathrm{H}), 2.28-2.45(\mathrm{~m}, 2 \mathrm{H})$, $1.26-2.03(\mathrm{~m}, 13 \mathrm{H}) ;{ }^{13} \mathrm{C} \mathrm{NMR}\left(\mathrm{CDCl}_{3}, 100 \mathrm{MHz}\right) \delta \mathrm{u}: 137.3,123.5,107.7,96.0,82.9$, $64.5,64.1,45.6,39.5,39.4,34.0,30.0,29.0,27.3,23.0$; d: 133.7, 131.6, 129.3, 129.1, 128.1, 127.8, 64.0, 44.6; HRMS calcd for $\mathrm{C}_{27} \mathrm{H}_{30} \mathrm{O}_{4} \mathrm{~S}\left(\mathrm{M}^{+}\right)$450.1865, found 450.1865. 


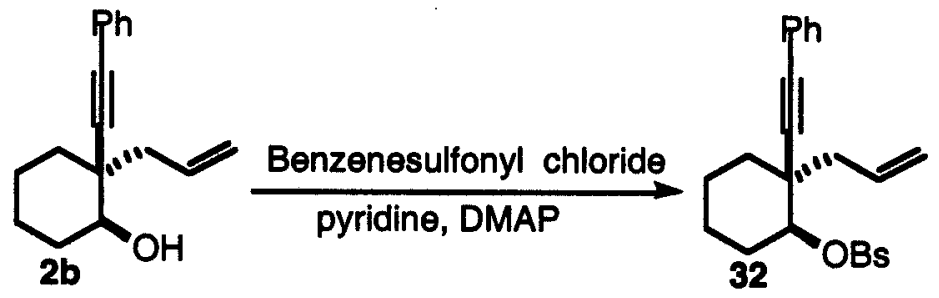

\section{Benzenesulfonate 32}

Same procedure as for the preparation of benzenesulfonate 29. Clear oil (91\% yield), TLC $R_{f}=0.62$ (PE:MTBE, 3:1). IR (neat, $\mathrm{cm}^{-1}$ ): 2940 (s), 1490 (w), 1448 (m), 1365 (s), 1186 (s); ${ }^{1} \mathrm{H}$ NMR (400 MHz, $\mathrm{CDCl}_{3}$ ) $\delta$ 7.92-7.96 (m, 2H), 7.49-7.64 (m, 3H), 7.25-7.41 $(\mathrm{m}, 5 \mathrm{H}), 5.88(\mathrm{~m}, 1 \mathrm{H}), 5.03-5.12(\mathrm{~m}, 2 \mathrm{H}), 4.39(\mathrm{dd}, \mathrm{J}=11.2 \mathrm{~Hz}, 4.4 \mathrm{~Hz}, 1 \mathrm{H}), 2.60(\mathrm{dd}$ $\mathrm{J}=13.6 \mathrm{~Hz}, 5.2 \mathrm{~Hz}, 1 \mathrm{H}), 2.02(\mathrm{dd}, \mathrm{J}=13.6 \mathrm{~Hz}, 8.4 \mathrm{~Hz}, 1 \mathrm{H}), 1.20-2.00(\mathrm{~m}, 8 \mathrm{H}) ;{ }^{13} \mathrm{C}$ NMR $\left(\mathrm{CDCl}_{3}, 100 \mathrm{MHz}\right) \delta \mathrm{u}: 138.1,123.7,118.7,89.9,85.8,43.0,42.5,36.0,29.9,24.5,21.9$; d:133.7, 133.5, 131.9, 129.3, 128.3, 128.1, 127.7, 86.3; HRMS calcd for $\mathrm{C}_{23} \mathrm{H}_{24} \mathrm{O}_{3} \mathrm{NaS}$ $(\mathrm{M}+\mathrm{Na})$ 403.1344, found 403.1333.

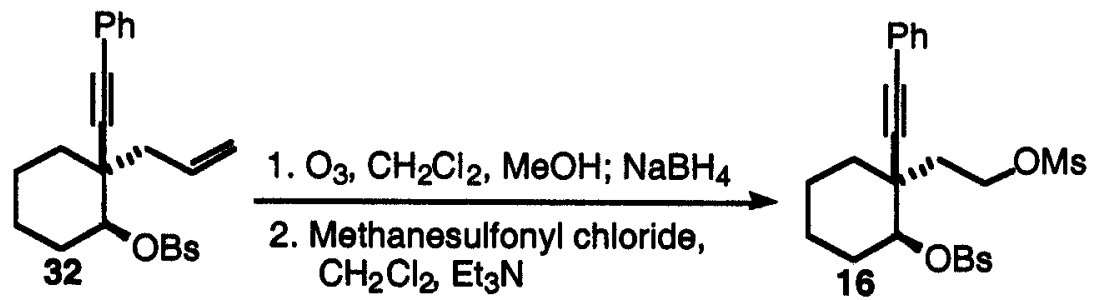

\section{Methanesulfonate 16}

Same procedure as for the preparation of methanesulfonate 13. Clear oil ( $85 \%$ yield), TLC $R_{f}=0.14$ (PE:MTBE, 1:1). IR (neat, $\mathrm{cm}^{-1}$ ): 2940 (s), 1448 (m), 1357 (s), 1176 (s); ${ }^{1} \mathrm{H}$ NMR (400 MHz, $\left.\mathrm{CDCl}_{3}\right) \delta 7.94(\mathrm{~d}, \mathrm{~J}=7.2 \mathrm{~Hz}, 2 \mathrm{H}), 7.65(\mathrm{t}, \mathrm{J}=7.2 \mathrm{~Hz}, 1 \mathrm{H}), 7.53-7.57$ (m, 2H), 7.26-7.41 (m, 5H), 4.44-4.53 (m, 2H), 4.39 (dd, J=11.2 Hz, 4.0 Hz, 1H), 2.99 (s, 
$3 \mathrm{H}), 1.26-2.31(\mathrm{~m}, 10 \mathrm{H}) ;{ }^{13} \mathrm{C} \mathrm{NMR}\left(\mathrm{CDCl}_{3}, 100 \mathrm{MHz}\right) \delta \mathrm{u}: 137.5,122.8,87.9,86.9,67.3$, $41.8,37.5,36.9,29.5,24.2,21.7$; d: $133.9,131.8,129.4,128.4,128.3,127.6,86.2,37.4$; HRMS calcd for $\mathrm{C}_{23} \mathrm{H}_{26} \mathrm{O}_{6} \mathrm{NaS}_{2}(\mathrm{M}+\mathrm{Na}) 485.1069$, found 485.1083.

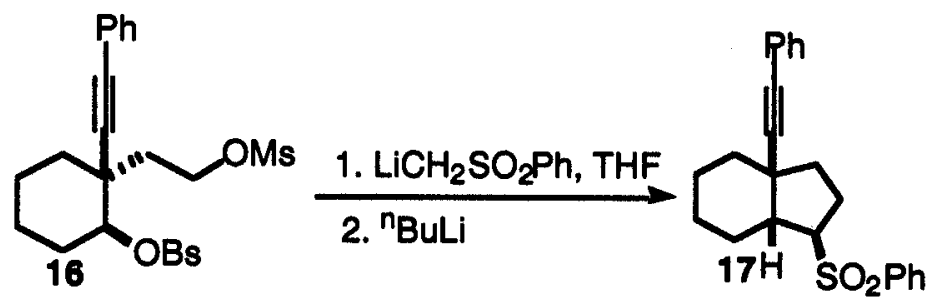

\section{Sulfone 17}

Same procedure as for the preparation of sulfone 3. White solid: $\mathrm{mp}=119-120^{\circ} \mathrm{C}(85 \%$ yield), TLC $R_{f}=0.59$ (PE:MTBE, 1:1). IR (film, $\mathrm{cm}^{-1}$ ): 2933 (s), 1489 (m), 1446 (s), 1302 (s), 1146 (s), 1086 (m); ${ }^{1} \mathrm{H}$ NMR (400 MHz, $\left.\mathrm{CDCl}_{3}\right)$ 8 7.90-7.94 (m, 2H), 7.54$7.68(\mathrm{~m}, 3 \mathrm{H}), 7.26-7.42(\mathrm{~m}, 5 \mathrm{H}), 3.55(\mathrm{~m}, 1 \mathrm{H}), 2.70(\mathrm{~m}, 1 \mathrm{H}), 1.19-2.30(\mathrm{~m}, 12 \mathrm{H}) ;{ }^{13} \mathrm{C}$ $\operatorname{NMR}\left(\mathrm{CDCl}_{3}, 100 \mathrm{MHz}\right) \delta$ u: $138.8,123.7,94.1,82.0,42.6,38.5,32.6,24.8,24.0,22.6$, 20.4; d: 133.8, 131.8, 129.4, 128.7, 128.3, 127.9, 63.7, 45.9; HRMS calcd for $\mathrm{C}_{23} \mathrm{H}_{24} \mathrm{O}_{2} \mathrm{~S}$ $\left(M^{+}\right)$364.1497, found 364.1513.

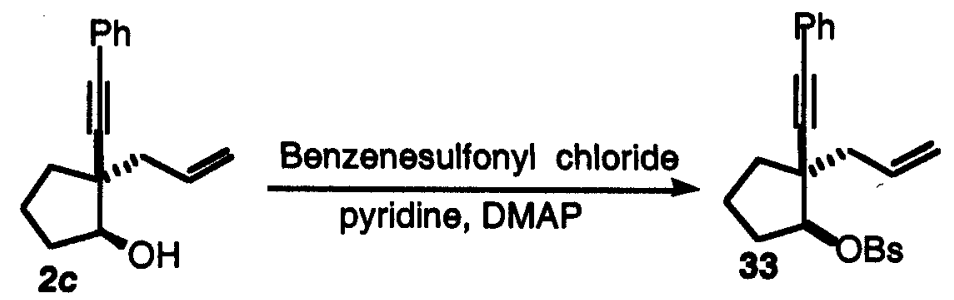

\section{Benzenesulfonate 33}

Same procedure as for the preparation of benzenesulfonate 29. Clear oil ( $92 \%$ yield), TLC $R_{f}=0.65$ (PE:MTBE, 3:1). IR (neat, $\mathrm{cm}^{-1}$ ): $2976(\mathrm{~s}), 1641$ (w), $1490(\mathrm{~m}), 1448(\mathrm{~m})$, 
1362 (s), 1176 (s); ${ }^{1} \mathrm{H}$ NMR (400 MHz, $\mathrm{CDCl}_{3}$ ) 8 7.89-7.93 (m, 2H), 7.38-7.56 (m, 3H), 7.25-7.35 (m, 5H), $5.85(\mathrm{~m}, 1 \mathrm{H}), 5.01-5.08(\mathrm{~m}, 2 \mathrm{H}), 4.61(\mathrm{t}, \mathrm{J}=5.6 \mathrm{~Hz}, 1 \mathrm{H}), 2.27(\mathrm{dd}$, $\mathrm{J}=14.0 \mathrm{~Hz}, 7.2 \mathrm{~Hz}, 1 \mathrm{H}), 2.10(\mathrm{dd}, \mathrm{J}=14.0 \mathrm{~Hz}, 7.2 \mathrm{~Hz}, 1 \mathrm{H}), 1.61-2.02(\mathrm{~m}, 6 \mathrm{H}) ;{ }^{13} \mathrm{C} N M R$ $\left(\mathrm{CDCl}_{3}, 100 \mathrm{MHz}\right) \delta \mathrm{u}: 137.2,123.5,118.4,90.2,84.5,47.1,41.9,35.5,30.4,20.1 ; \mathrm{d}:$ 133.7, 133.6, 131.8, 129.1, 128.2, 128.0, 127.9, 87.5; HRMS calcd for $\mathrm{C}_{22} \mathrm{H}_{22} \mathrm{O}_{3} \mathrm{NaS}$ $(\mathrm{M}+\mathrm{Na}) 389.1187$, found 389.1207 .

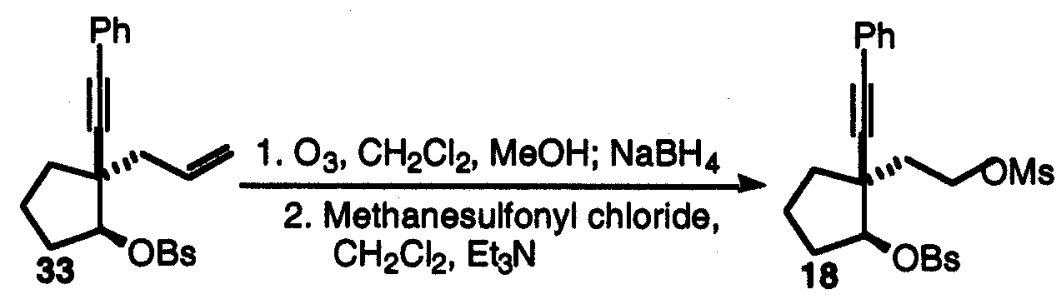

\section{Methanesulfonate 18}

Same procedure as for the preparation of methanesulfonate 13. Clear oil ( $84 \%$ yield), TLC $R_{f}=0.13\left(\right.$ PE:MTBE, 1:1). IR (neat, $\left.\mathrm{cm}^{-1}\right): 2960$ (s), 1449 (m), 1359 (s), 1175 (s); ${ }^{1} \mathrm{H} \mathrm{NMR}\left(400 \mathrm{MHz}, \mathrm{CDCl}_{3}\right)$ 8 7.90-7.94 (m, 2H), $7.61(\mathrm{~m}, 1 \mathrm{H})$, 7.48-7.52 (m, 2H), 7.27$7.39(\mathrm{~m}, 5 \mathrm{H}), 4.62(\mathrm{t}, \mathrm{J}=6.4 \mathrm{~Hz}, 1 \mathrm{H}), 4.46(\mathrm{t}, \mathrm{J}=6.8 \mathrm{~Hz}, 2 \mathrm{H}), 2.99(\mathrm{~s}, 3 \mathrm{H}), 1.63-2.17(\mathrm{~m}$, $8 \mathrm{H}) ;{ }^{13} \mathrm{C} \mathrm{NMR}\left(\mathrm{CDCl}_{3}, 100 \mathrm{MHz}\right) \delta \mathrm{u}: 136.8,122.8,88.0,85.8,67.6,45.8,36.4,36.3$, 29.1, 19.7; d: 133.8, 131.7, 129.3, 128.3, 127.7, 87.4, 37.2; HRMS calcd for $\mathrm{C}_{22} \mathrm{H}_{24} \mathrm{O}_{6} \mathrm{NaS}_{2}(\mathrm{M}+\mathrm{Na})$ 471.0912, found 471.0893. 


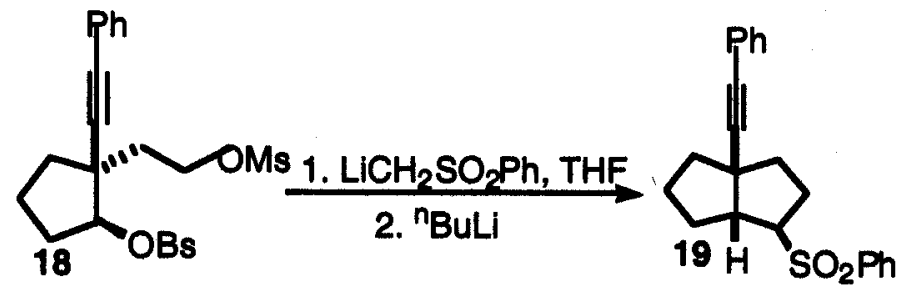

\section{Sulfone 19}

Same procedure as for the preparation of sulfone 3. Colorless crystals ( $86 \%$ yield): $\mathrm{mp}=$ $83-84^{\circ} \mathrm{C}$ (\% yield), TLC $R_{f}=0.58$ (PE:MTBE, 1:1). IR (neat, $\mathrm{cm}^{-1}$ ): $2958(\mathrm{~s}), 2868(\mathrm{~m})$, 2221 (w), 1490 (m), 1446 (s), 1302 (s), 1146 (s), 1086 (s); ${ }^{1} \mathrm{H}$ NMR (400 MHz, $\mathrm{CDCl}_{3}$ ) $\delta$ 7.90-7.94 (m, $2 \mathrm{H}), 7.53-7.64(\mathrm{~m}, 3 \mathrm{H}), 7.24-7.35(\mathrm{~m}, 5 \mathrm{H}), 3.01-3.11(\mathrm{~m}, 2 \mathrm{H}), 1.29-2.26$ $(\mathrm{m}, 10 \mathrm{H}) ;{ }^{13} \mathrm{C} \mathrm{NMR}\left(\mathrm{CDCl}_{3}, 100 \mathrm{MHz}\right) \delta \mathrm{u}: 138.5,123.8,96.6,80.9,49.3,40.7,39.6$, $33.1,28.8,25.8 ;$ d: $133.8,131.6,129.3,128.8,128.2,127.7,71.9,53.4$; HRM calcd for $\mathrm{C}_{22} \mathrm{H}_{22} \mathrm{O}_{2} \mathrm{~S}\left(\mathrm{M}^{+}\right)$350.1341, found 350.1348 .

\section{References:}

1. Still, W. C.; Kahn, M.; Mitra, A. J. Org. Chem. 1978, 43, 2923.

2. Taber, D. F. J. Org. Chem. 1982, 47, 1351.

3. Davies, M. T.; Dobson, D. F.; Hayman, D. F.; Jackman, G. B.; Lester, M. G.; Petrow, V.; Stephenson, O.; Webb, A. A.; Tetrahedron 1962, 18, 751.

4. The buffer solution is prepared by adding $4.5 \mathrm{~mL}$ of glacial acetic acid to $1 \mathrm{~L}$ of a $0.1 \mathrm{M}$ solution of $\mathrm{K}_{2} \mathrm{CO}_{3}$.

5. Tu, Y.; Frohn, M.; Wang, Z.; Shi, Y. Org. Syn. 2003, 80, 1.

6. Morisson V.; Barnier, J. P.; Blanco, L. Tetrahedron 1998, 54, 7749. 

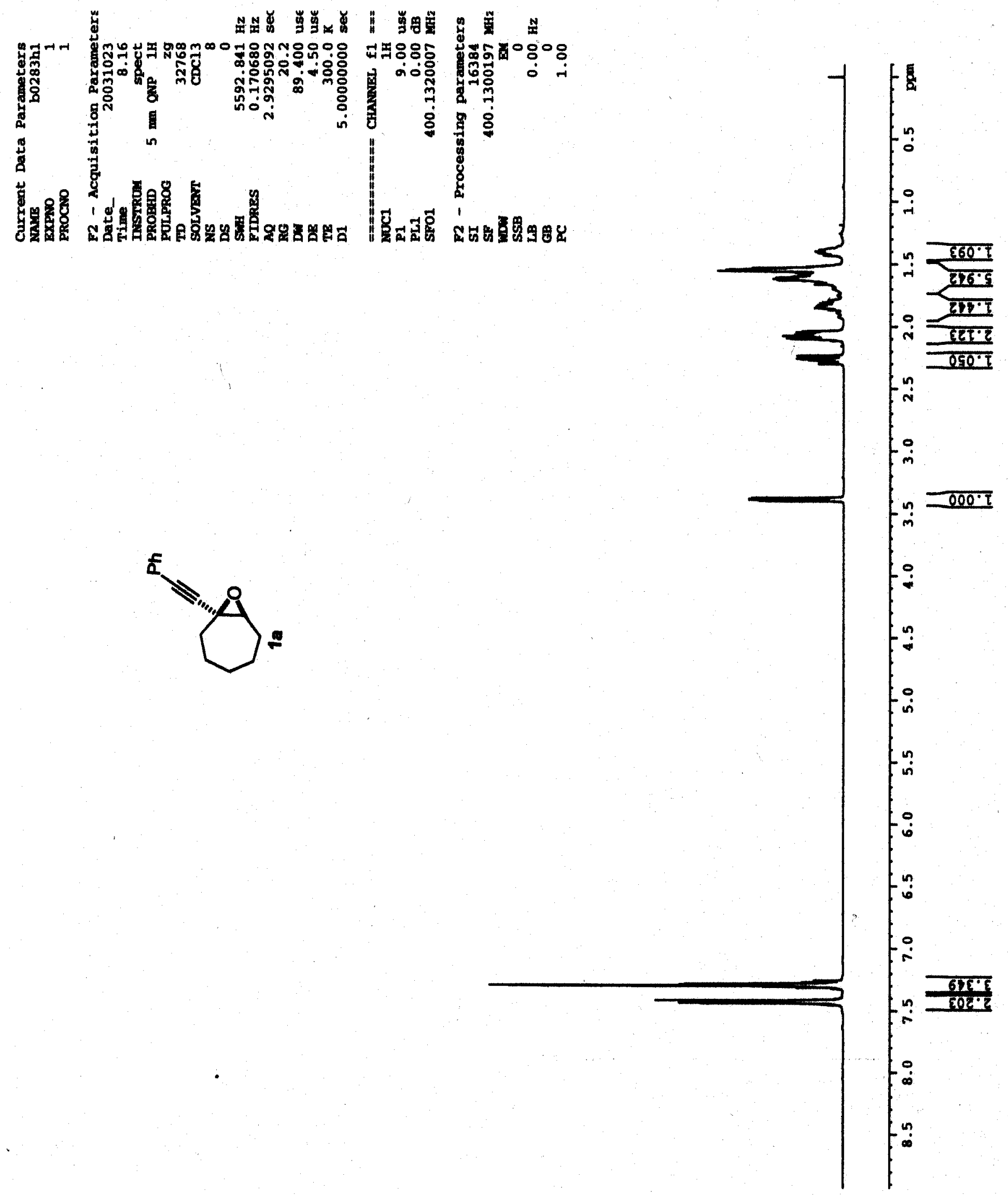

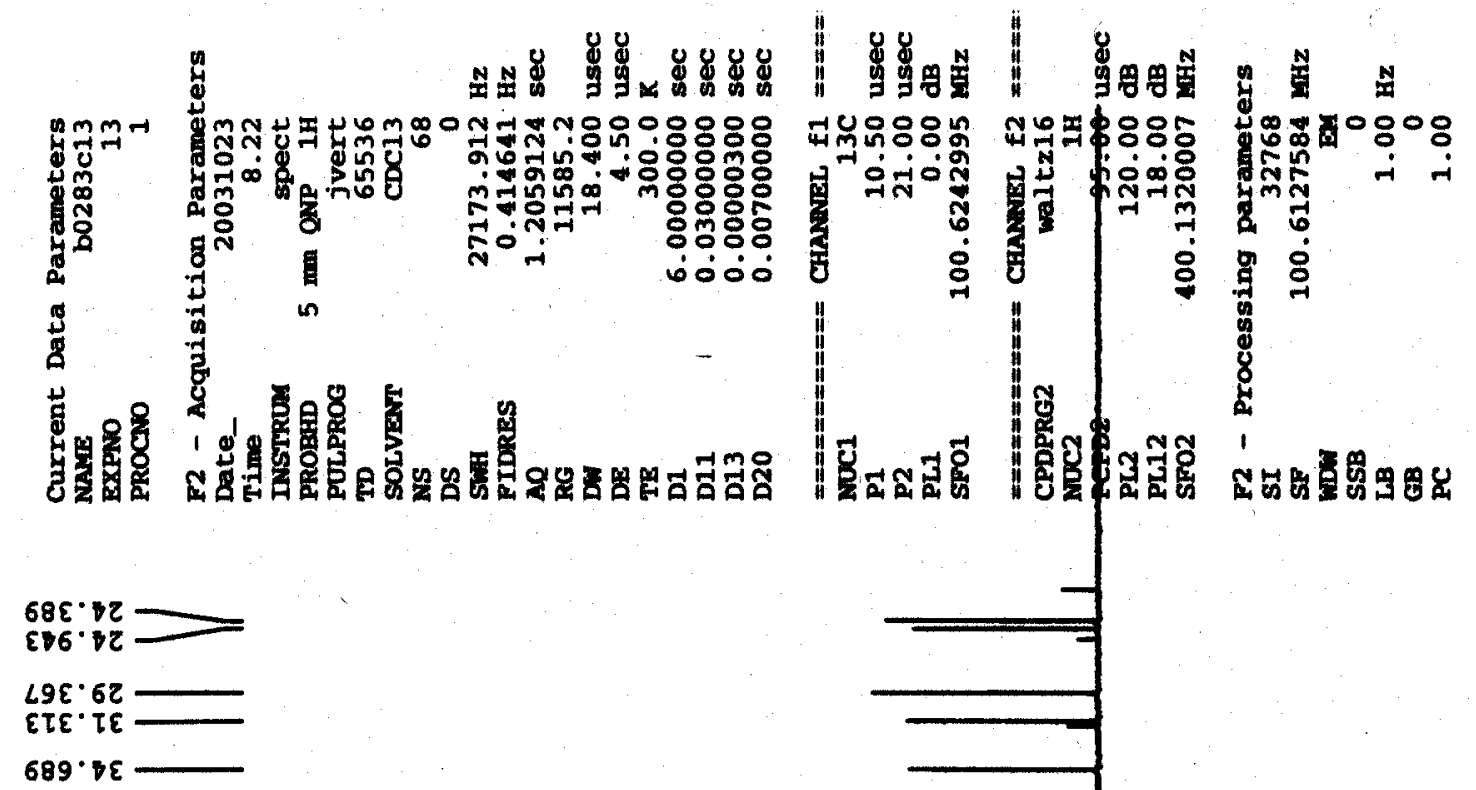

$22 L \cdot D S$

७९6. $89-$
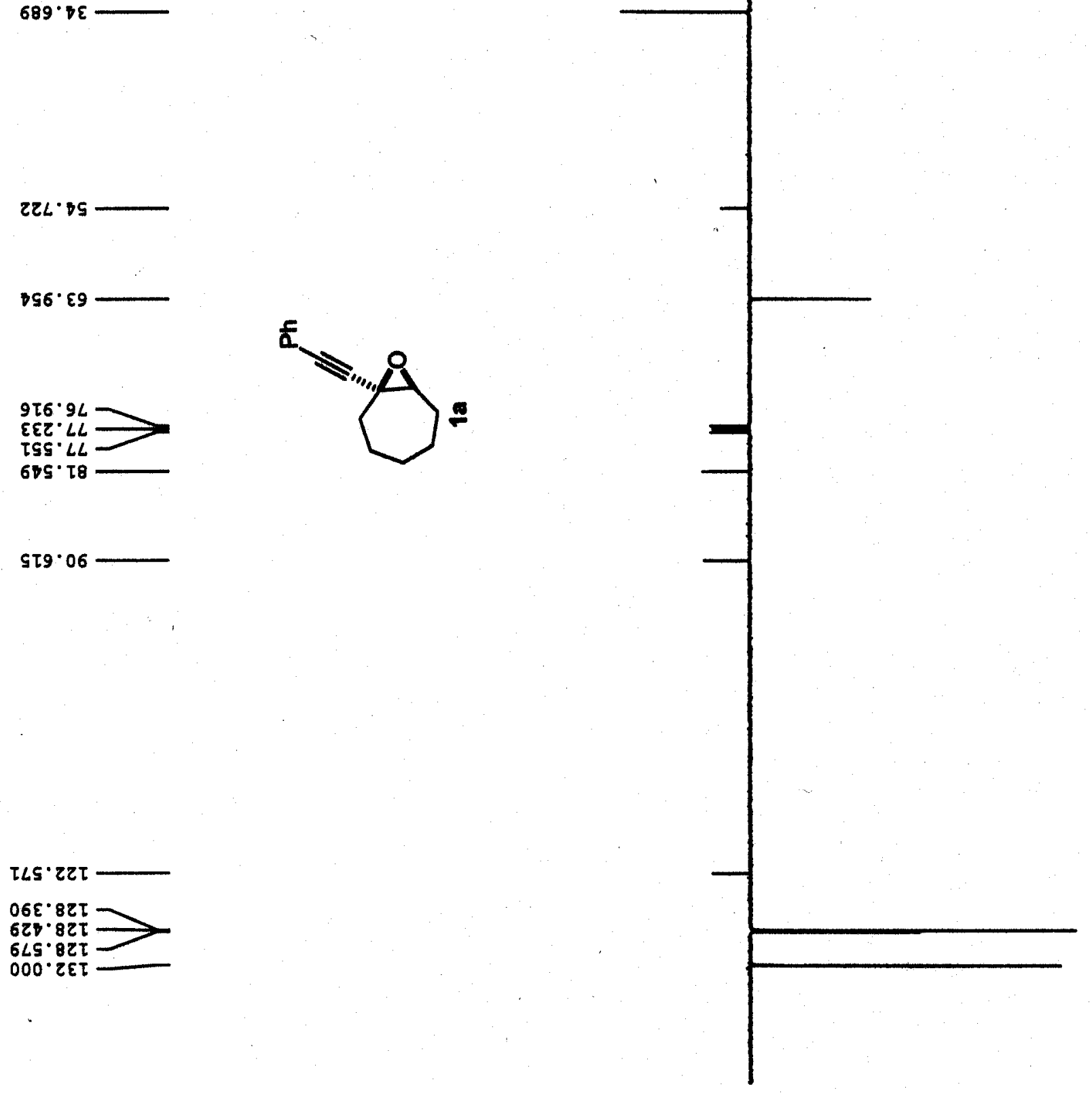

\$โ9.06 

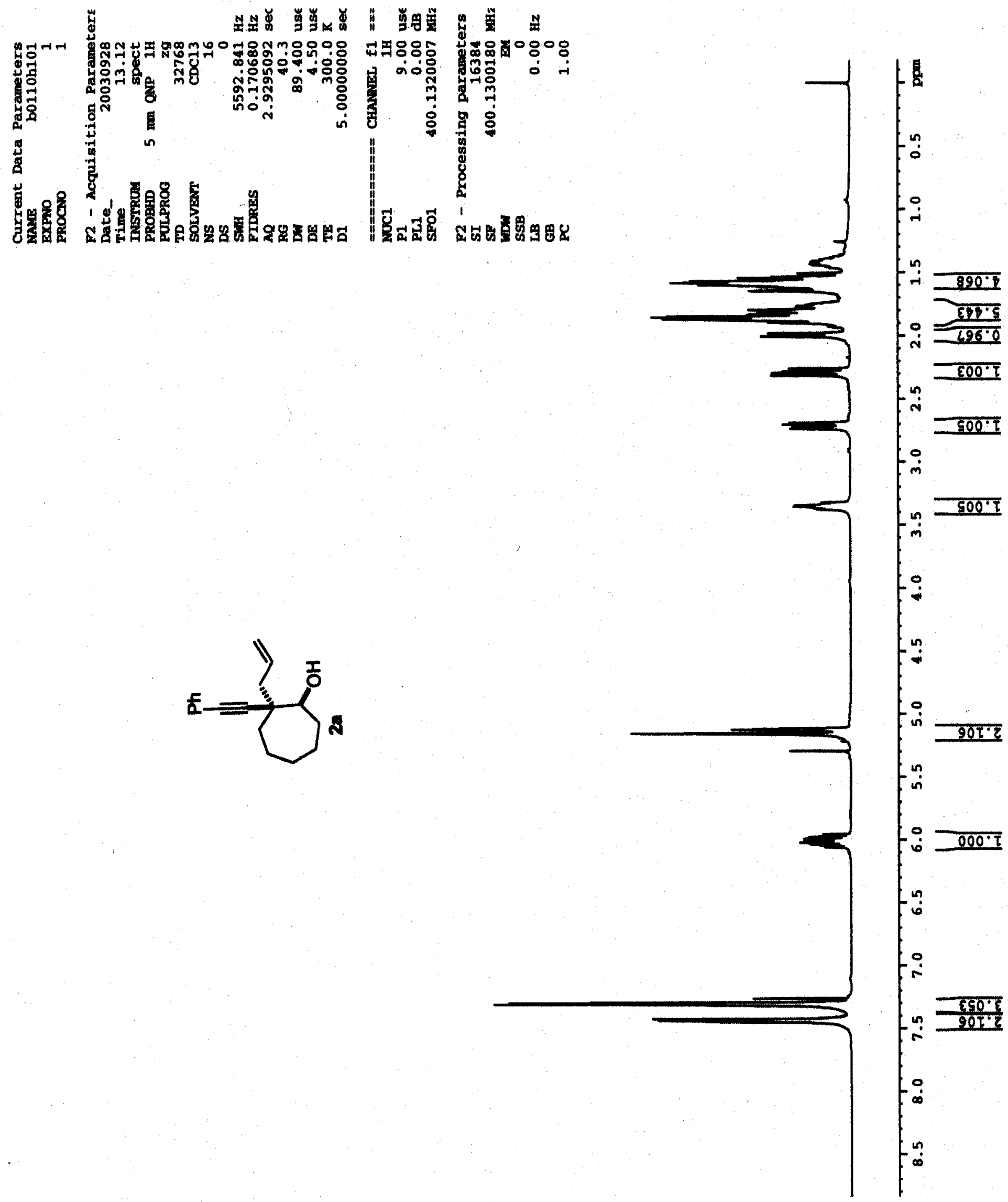


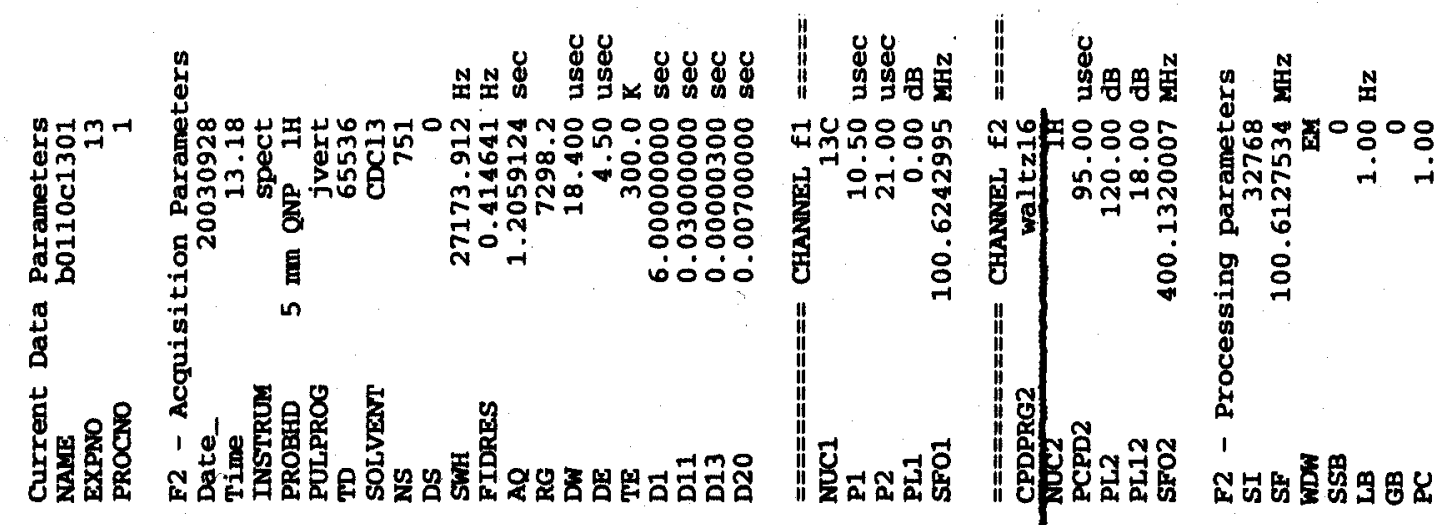

$\tau z \mathrm{z} \cdot \mathrm{z}$

126. $22 \square$

$800 . \angle Z$

$6 \angle 9^{\circ} \varepsilon \varepsilon$
$L L S^{\circ} \cdot \varepsilon$

โ9E.ด๐

\$\$O $\angle$

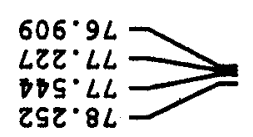

$675 \cdot 98 \longrightarrow$

$\angle \nabla S \cdot \tau 6$

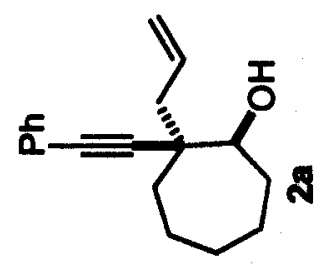

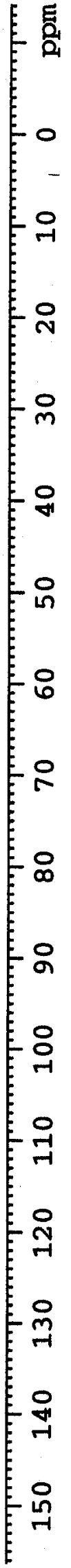


HPLC Condition:

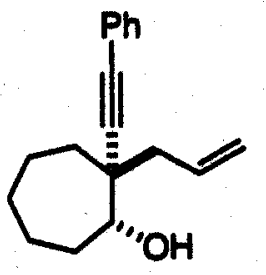

$(+)-2 a$
Column: Chiralcel OD

Chiral Technologies Inc.

Eluent: Hexane / Isopropanol (99 / 1)

Flow rate: $1.0 \mathrm{~mL} / \mathrm{min}$
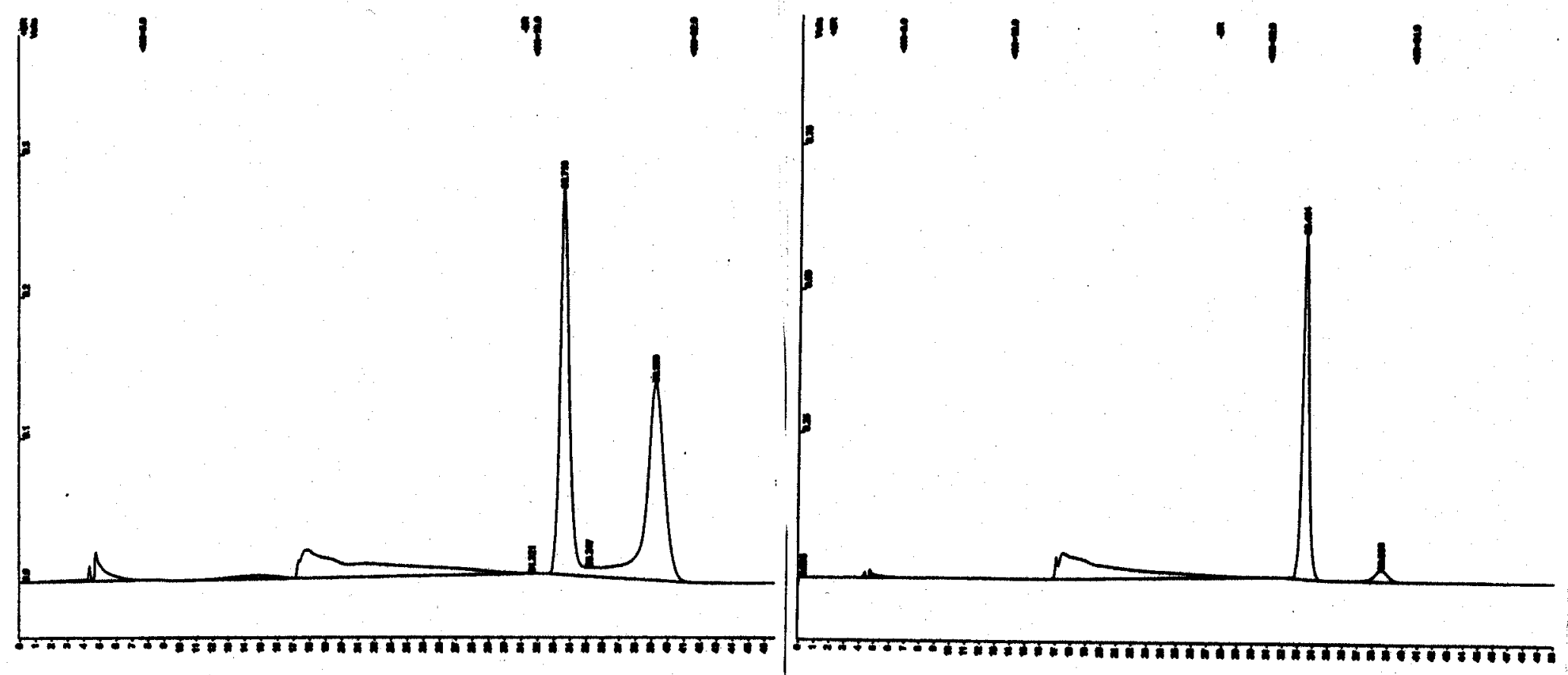

\begin{tabular}{cccc}
$\begin{array}{c}\text { Peak } \\
\text { No. }\end{array}$ & Result & $\begin{array}{c}\text { Ret. } \\
\text { Time } \\
(\mathrm{min})\end{array}$ & $\begin{array}{c}\text { Area } \\
\text { (Counts) }\end{array}$ \\
\hline 1 & 50.0668 & 33.713 & 11003448 \\
2 & 49.9120 & 39.339 & 10969420 \\
\hline \multicolumn{4}{c}{ Racemate }
\end{tabular}

\begin{tabular}{cccc}
$\begin{array}{c}\text { Peak } \\
\text { No. }\end{array}$ & Result & $\begin{array}{c}\text { Ret. } \\
\text { Time } \\
\text { (min) }\end{array}$ & $\begin{array}{c}\text { Area } \\
\text { (Counts) }\end{array}$ \\
\hline 1 & 93.0117 & 33.494 & 18160704 \\
2 & 6.8553 & 38.630 & 1338514 \\
\hline
\end{tabular}

Reaction matorial 

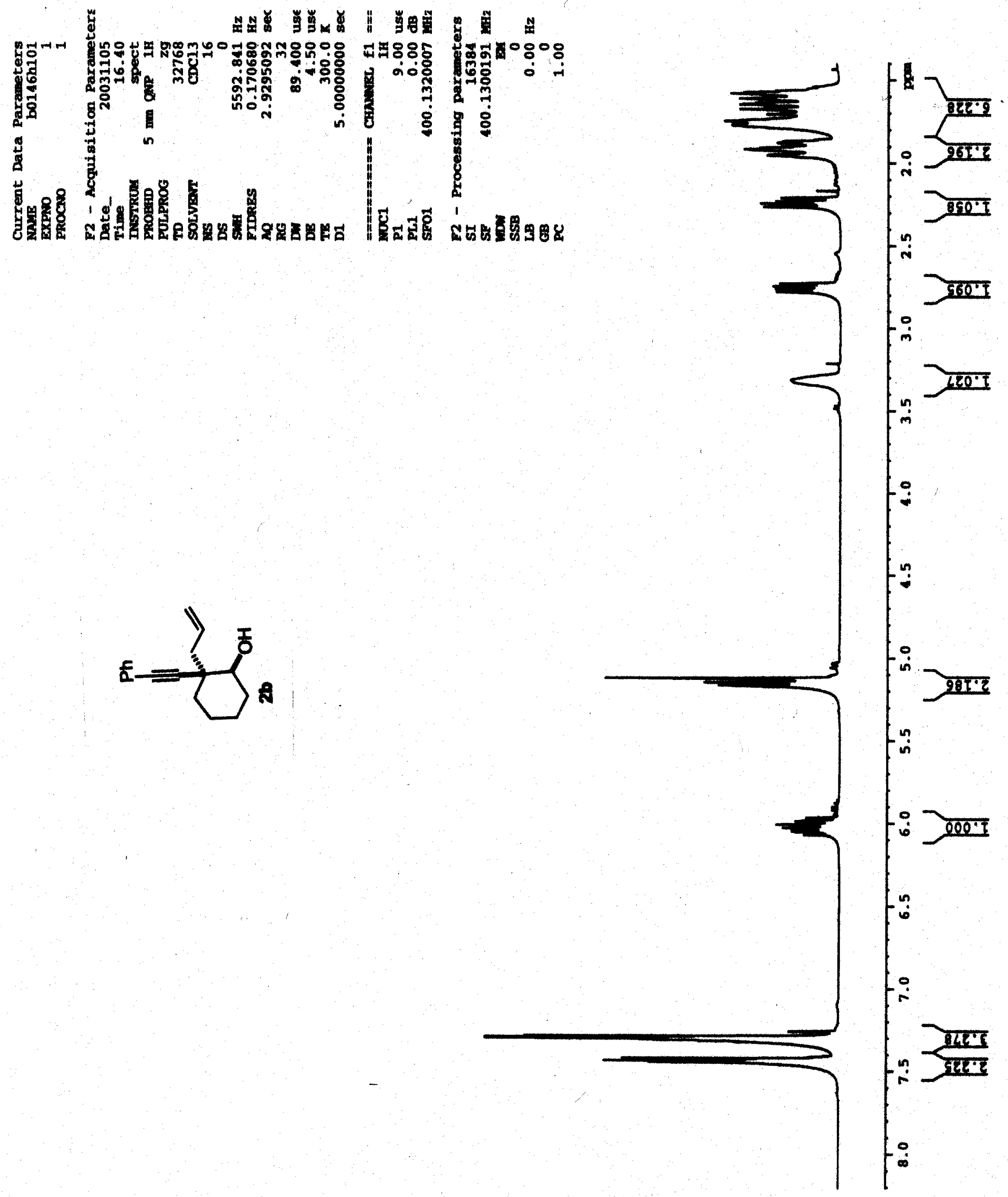


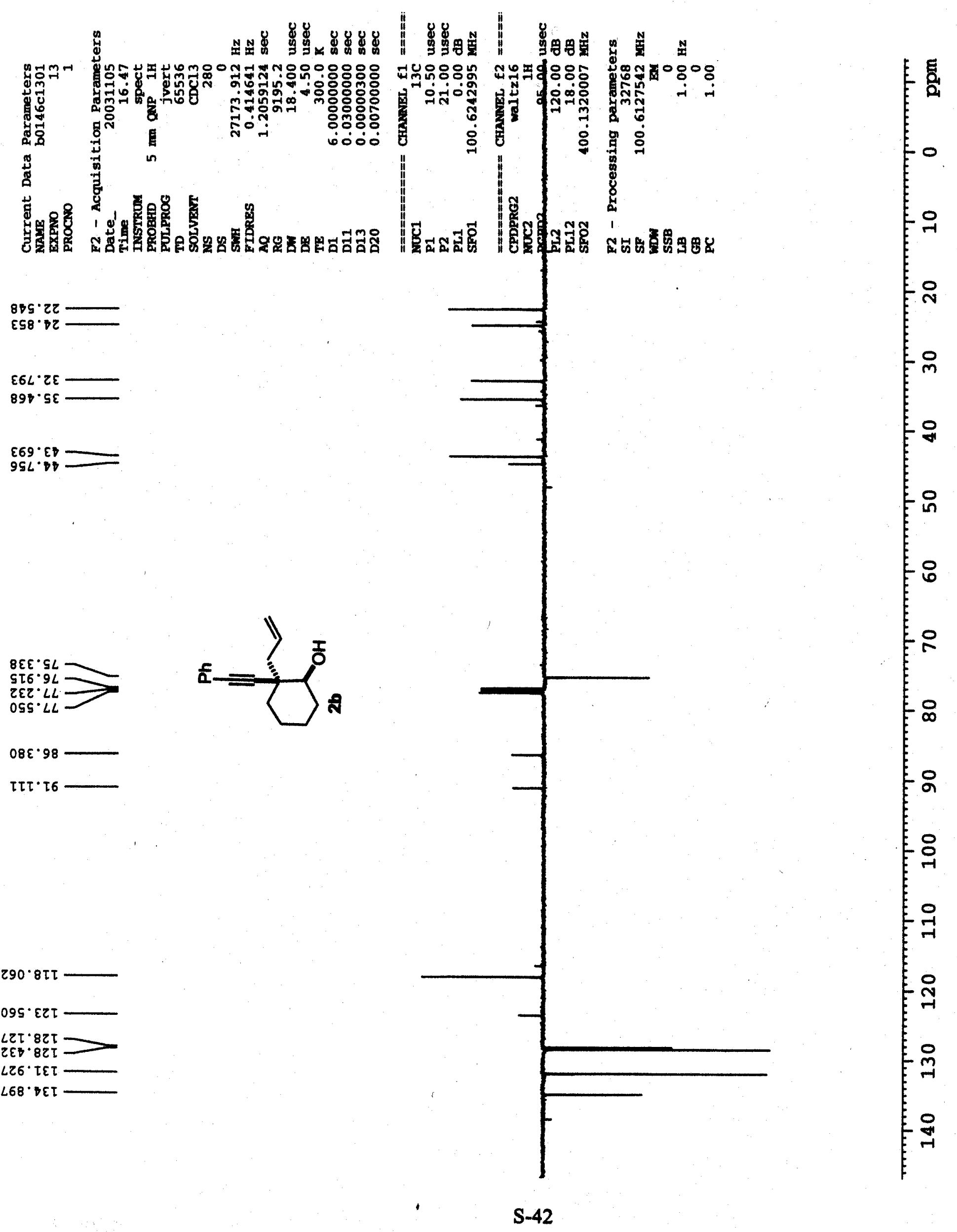



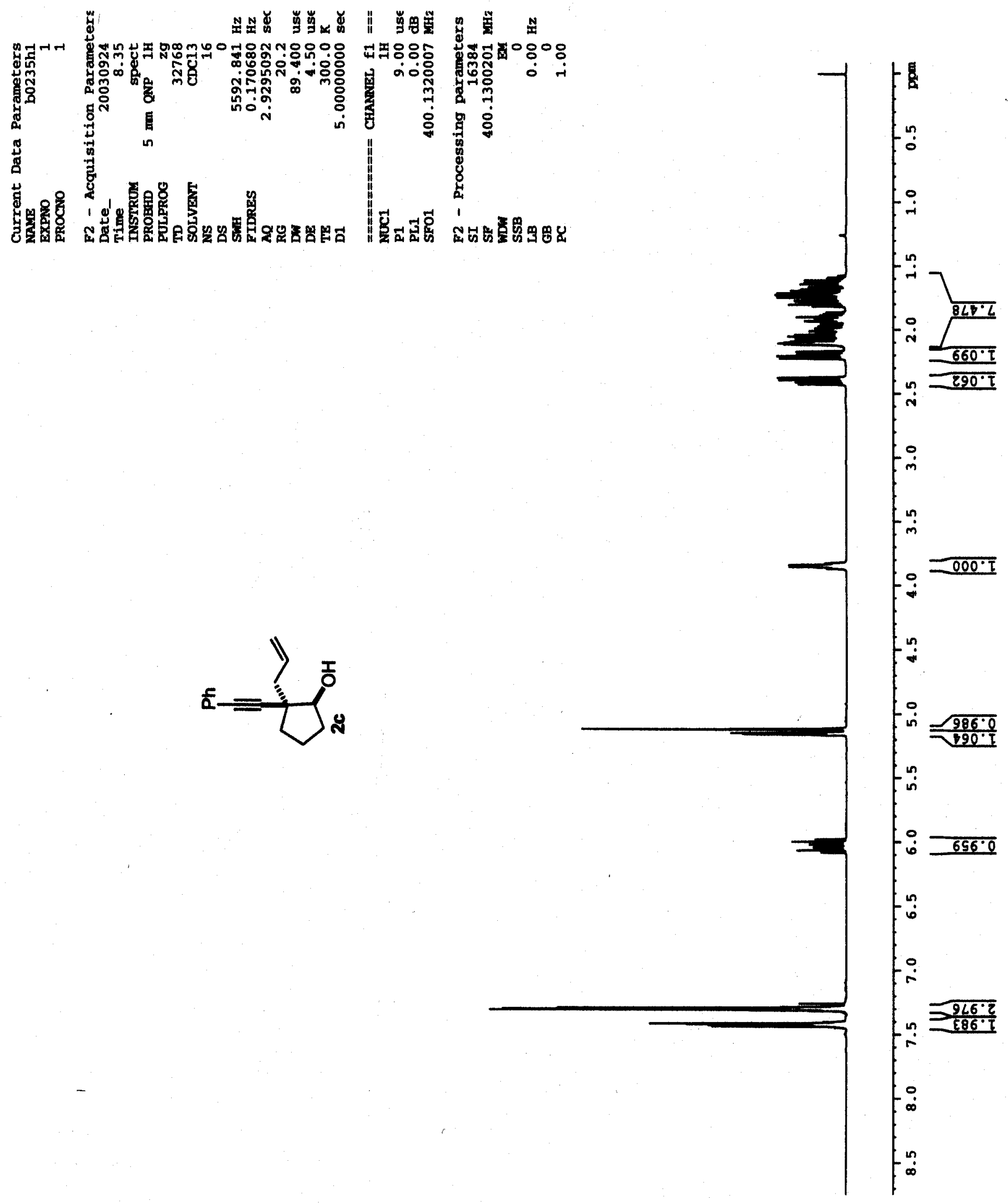


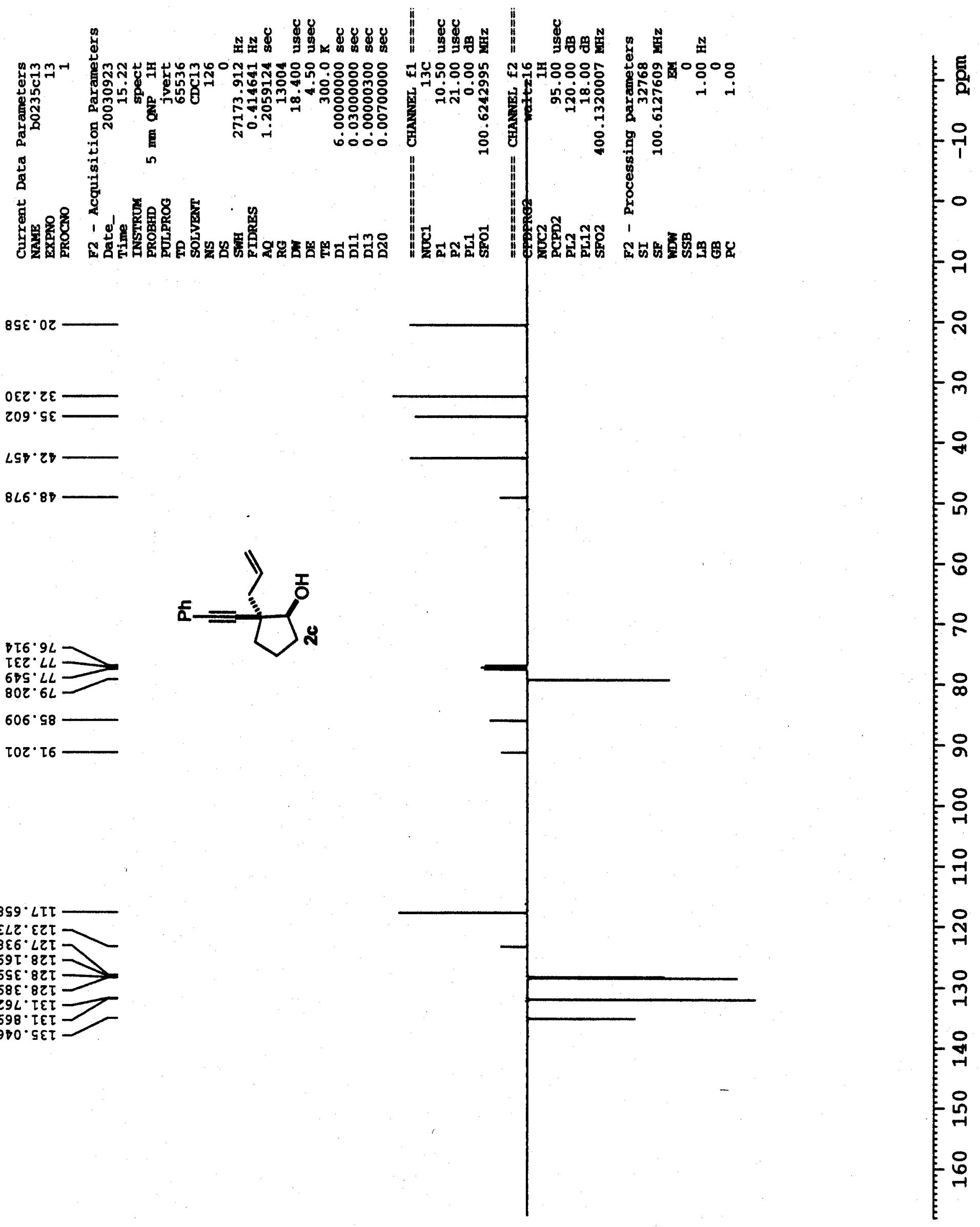



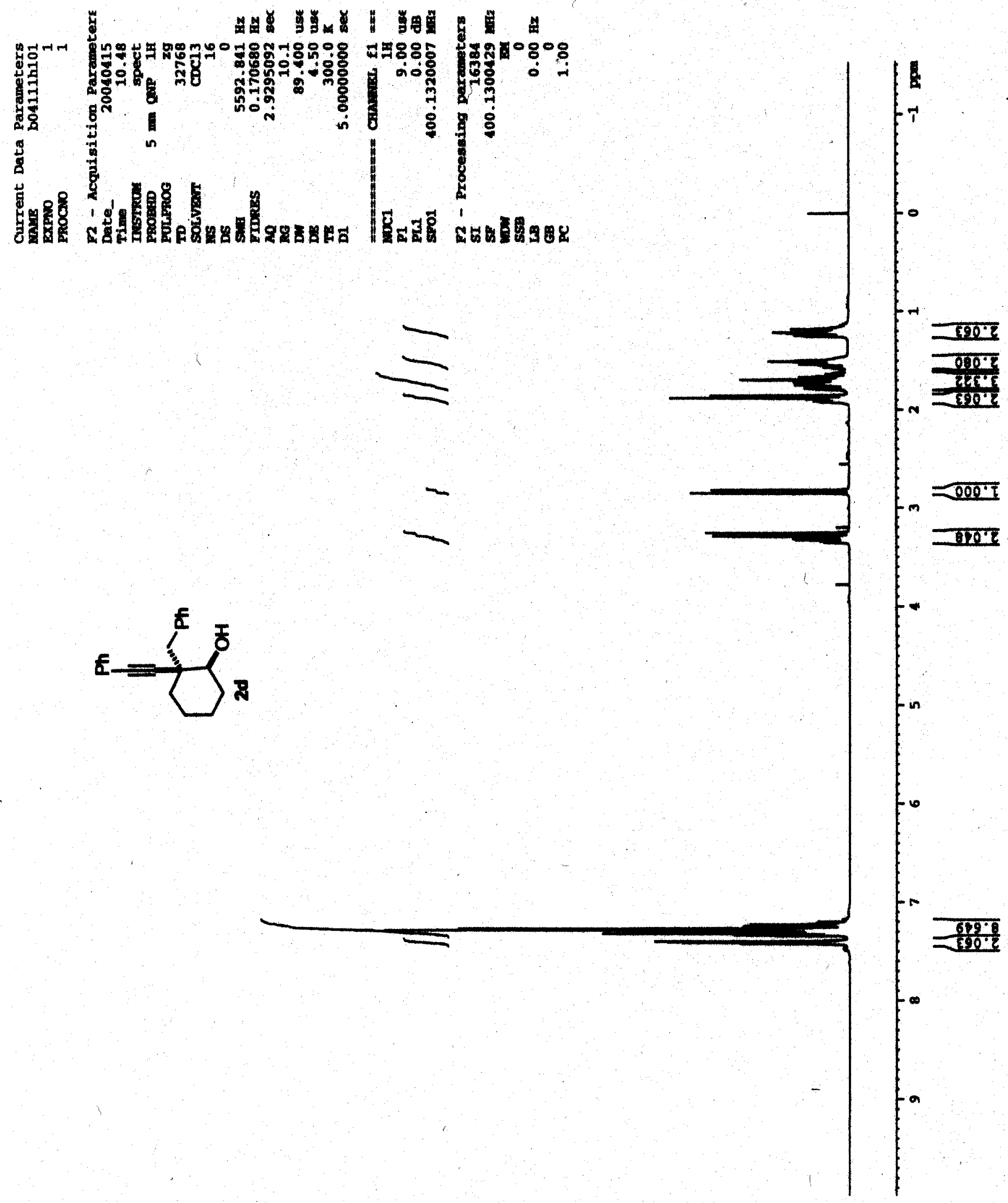

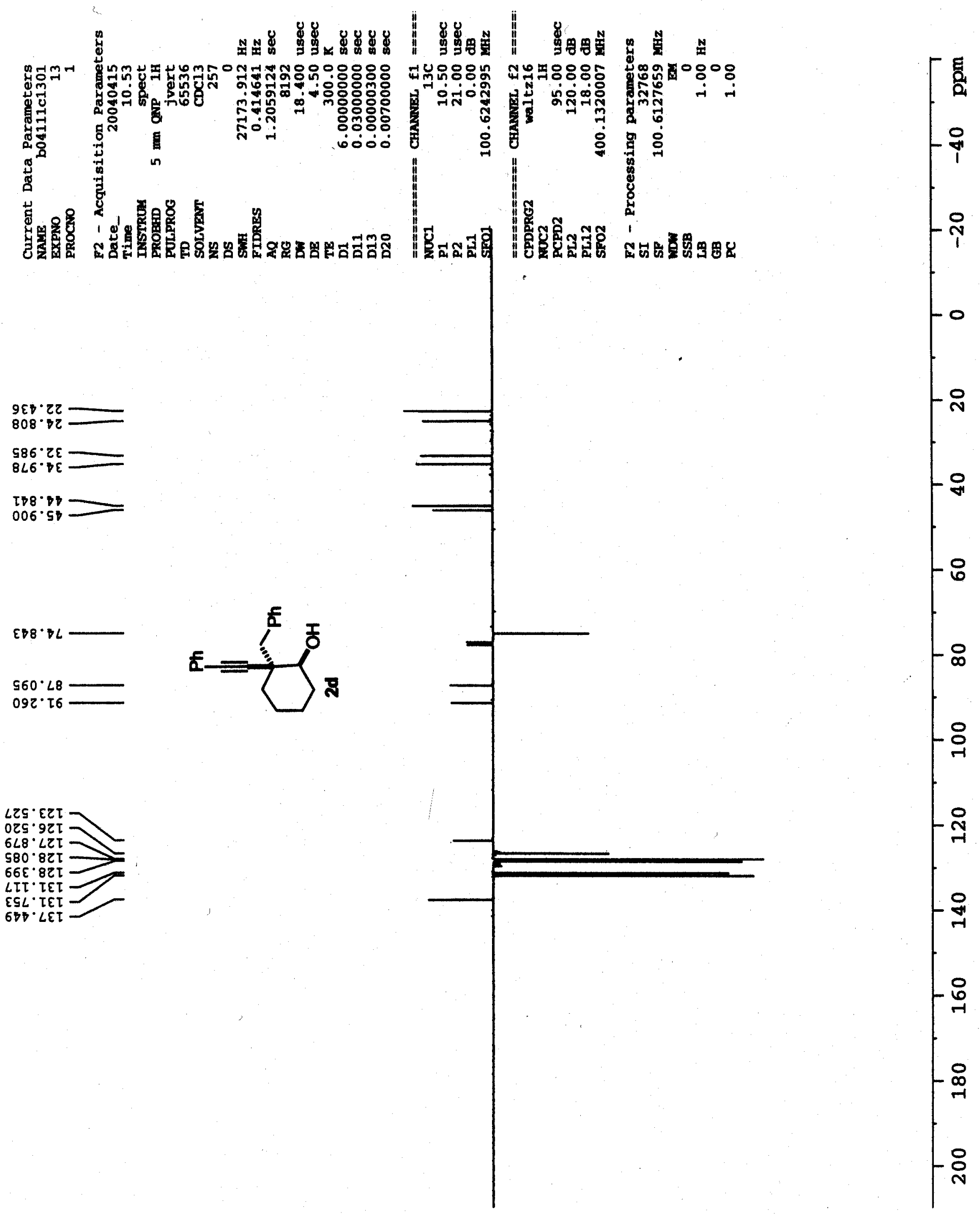

$\varepsilon \triangleright 8^{\circ} \cdot \mathcal{L}$

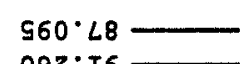

$092 \cdot \tau 6$
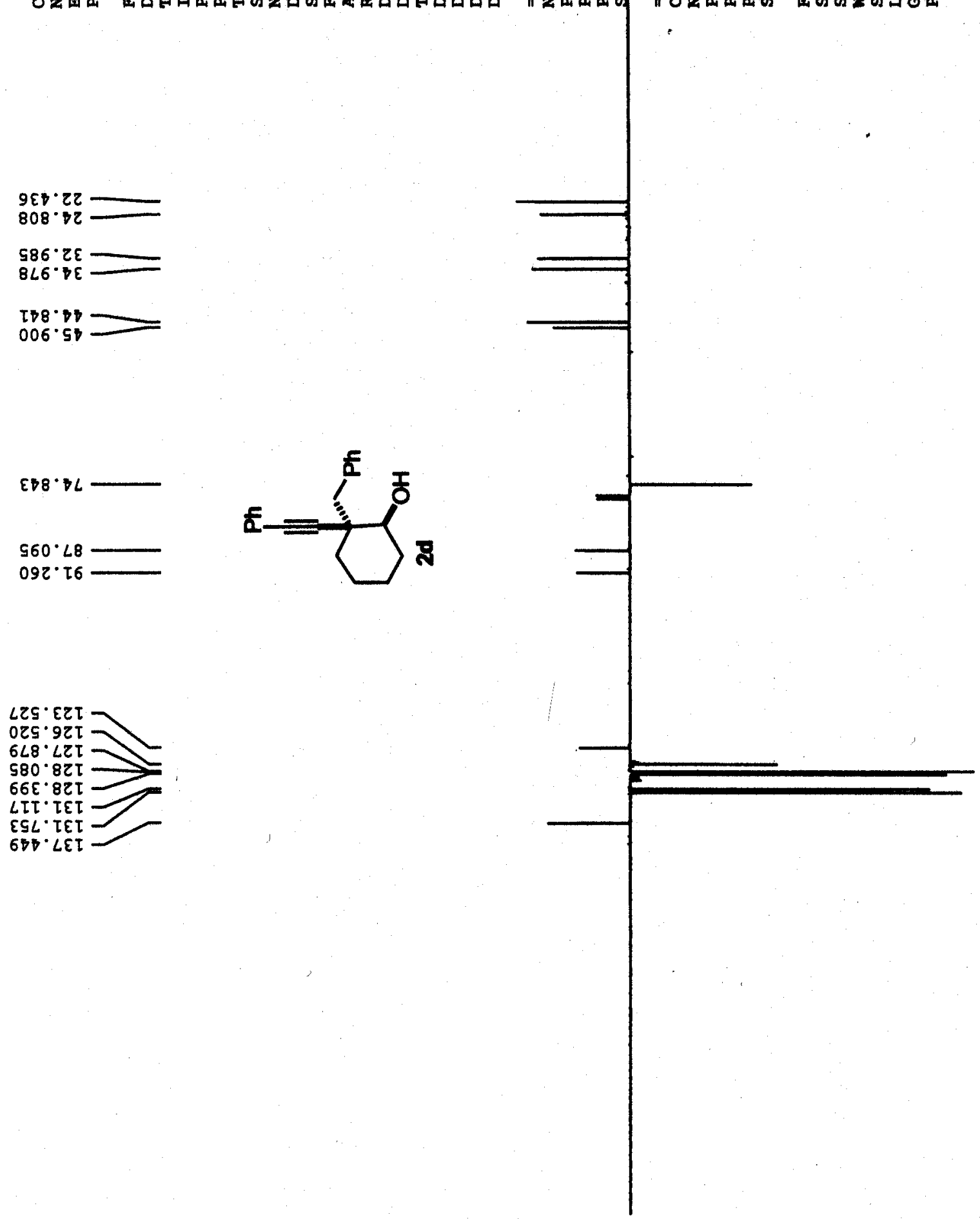

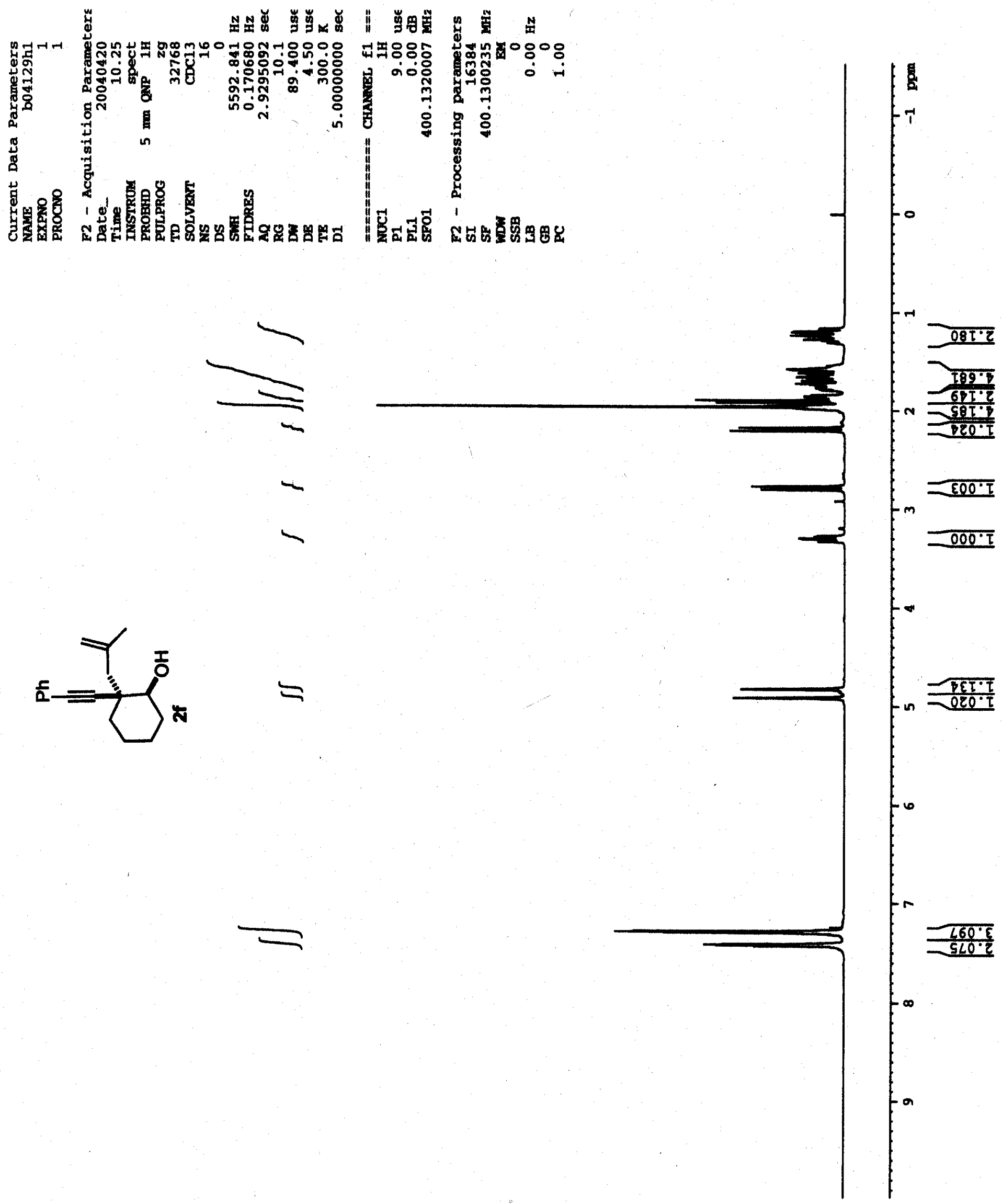

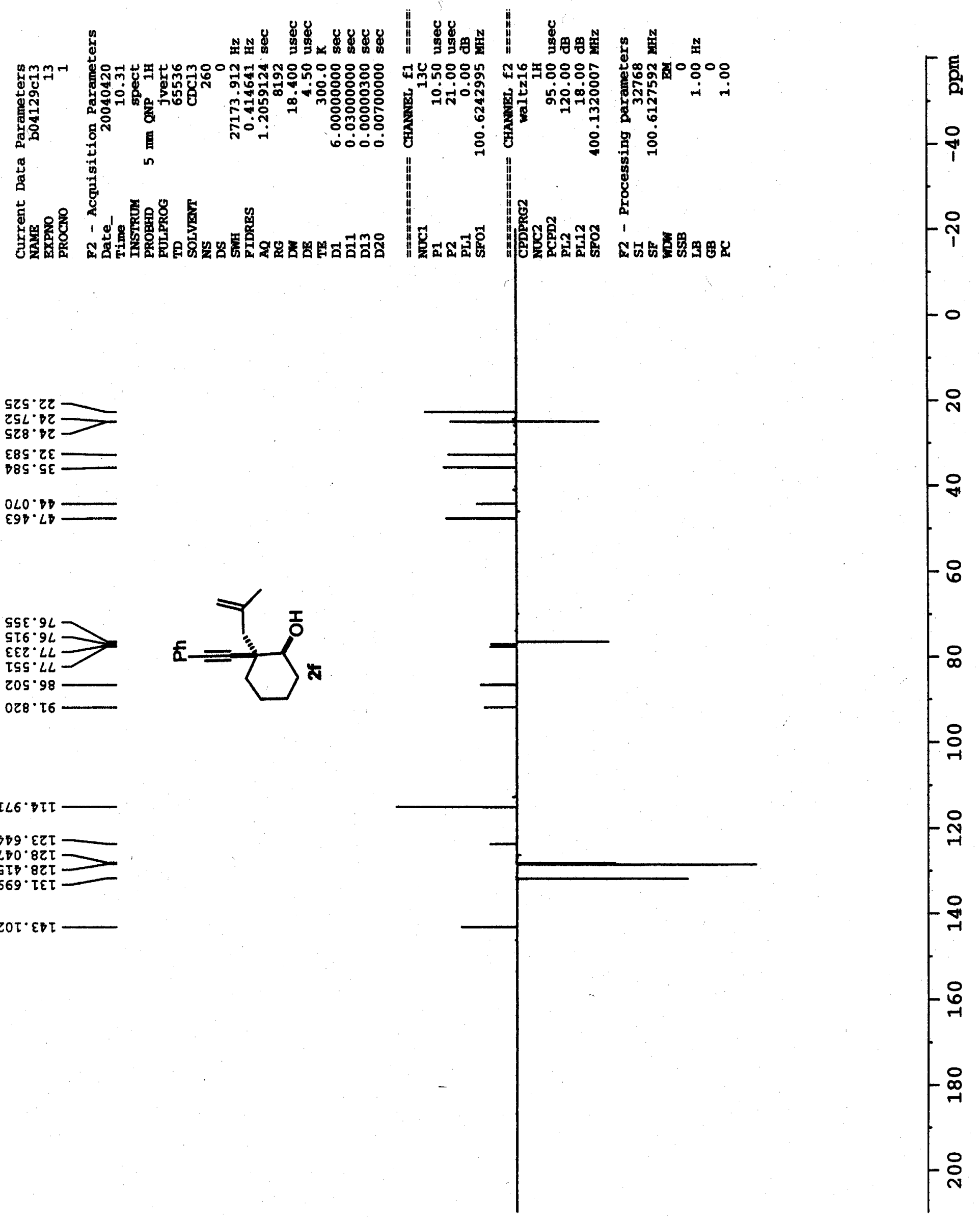

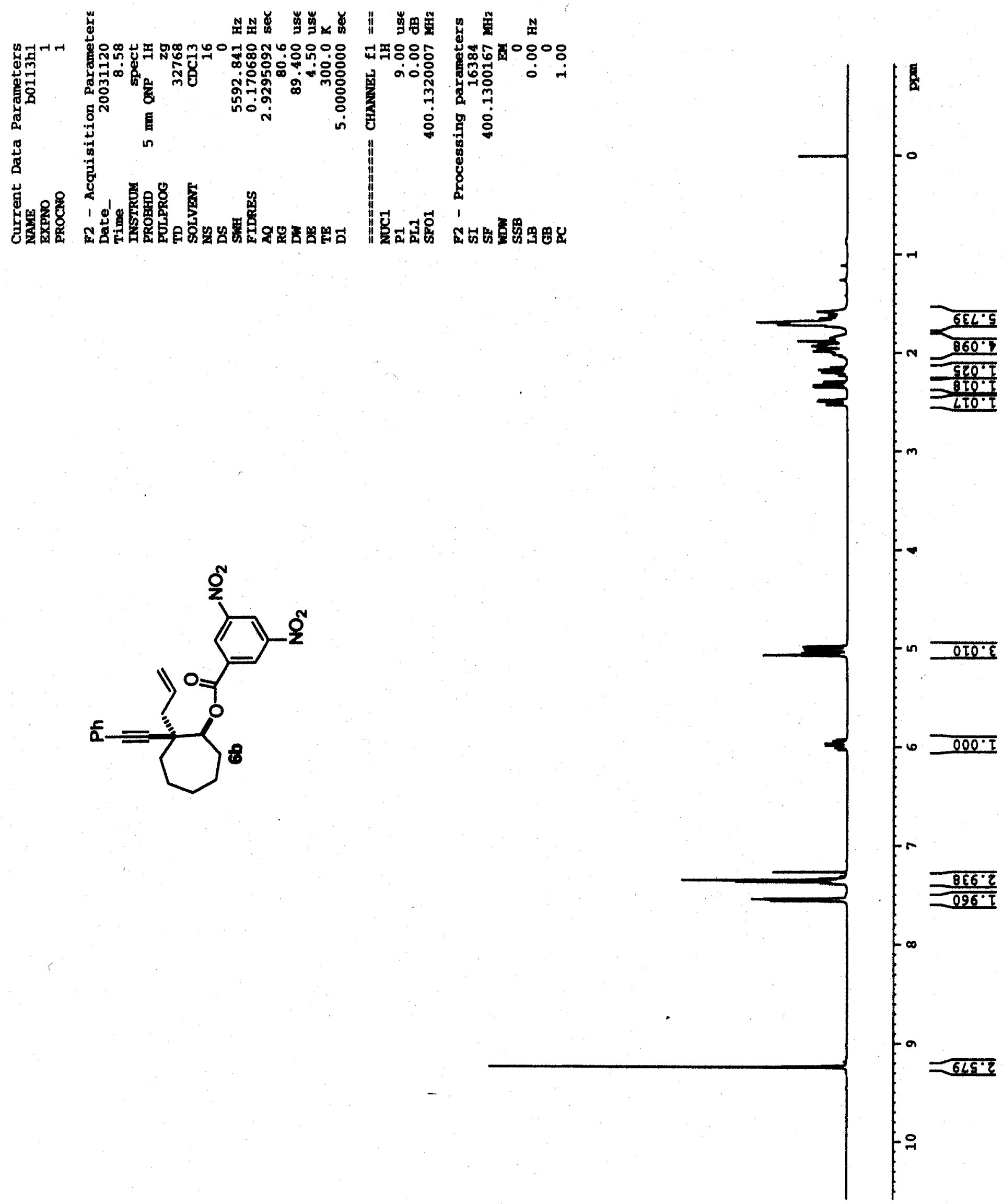


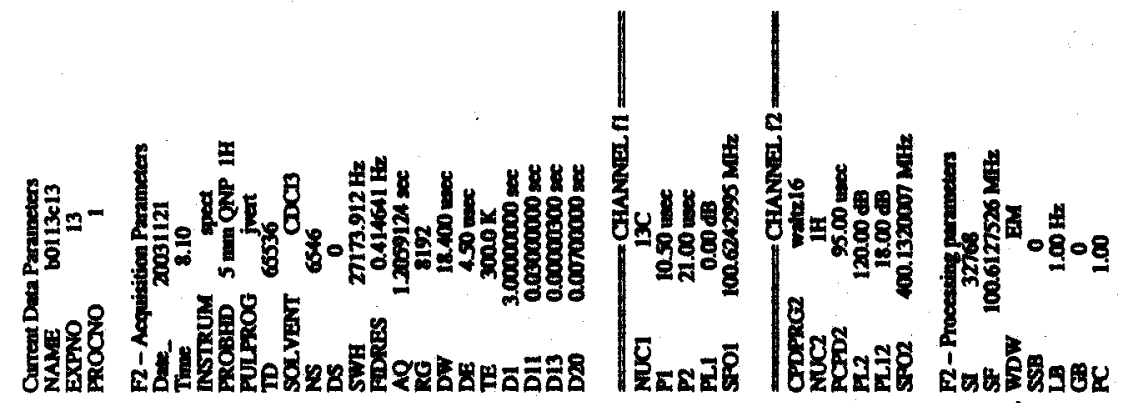

$\angle Z I ' Z 2$

$2 \$ 9.22$

$\downarrow t<\cdot 92$

$8 \pitchfork \tau \cdot 1 \varepsilon$

$\varepsilon \tau L \downarrow \downarrow \varepsilon$

$2 t s^{\circ}+t=$
$\left[t 0^{\circ} s t=\right.$

$0169 L$

$8 Z 2 \angle L$

StS LL

ع09.28

$\$ 82$ ' 58

$\$ 2606$
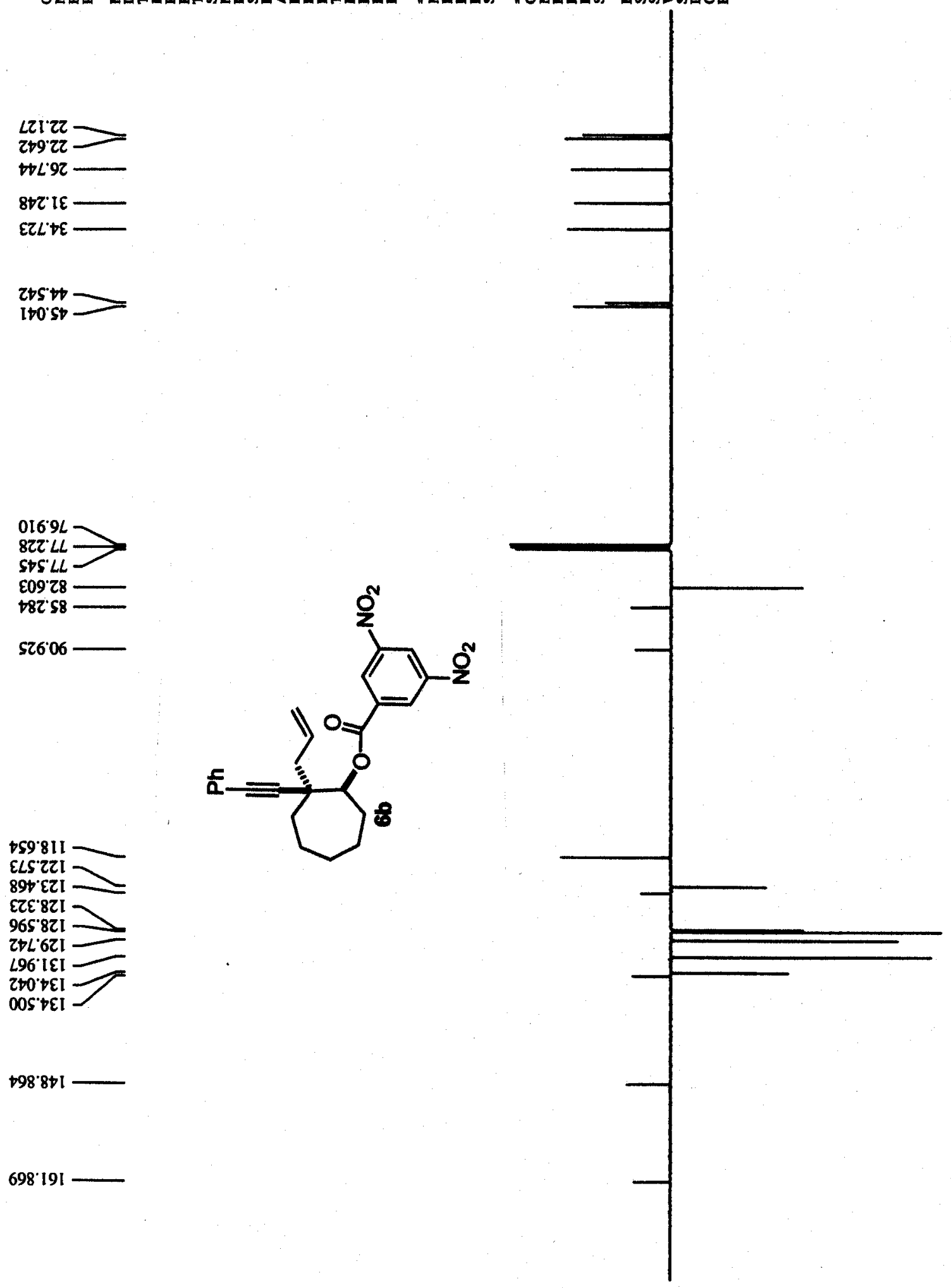

Tomen

$+98.8 \mathrm{tl}$

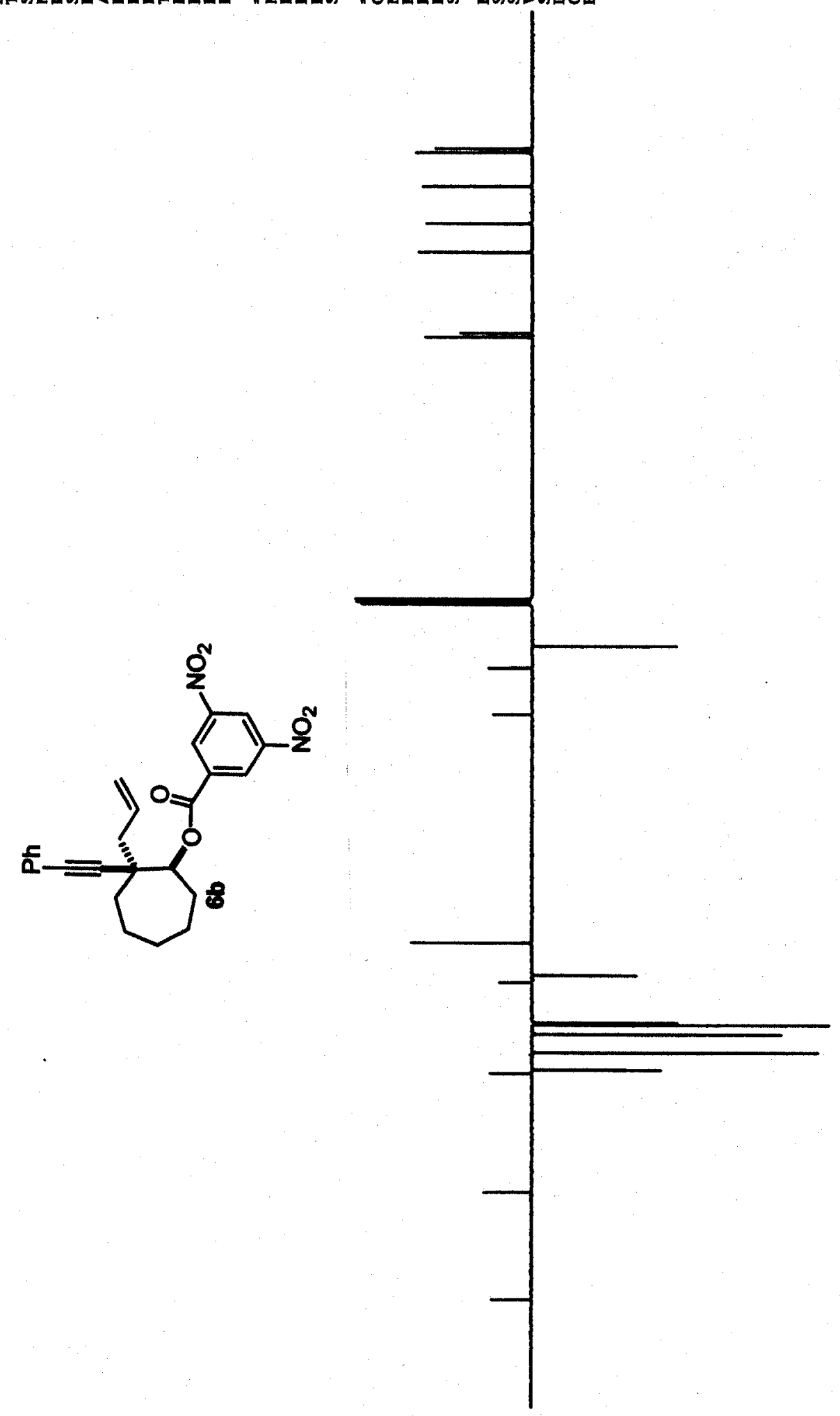

698' 191

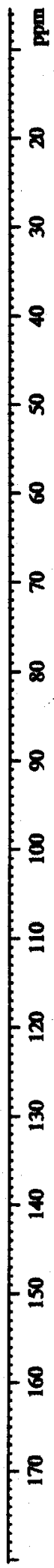



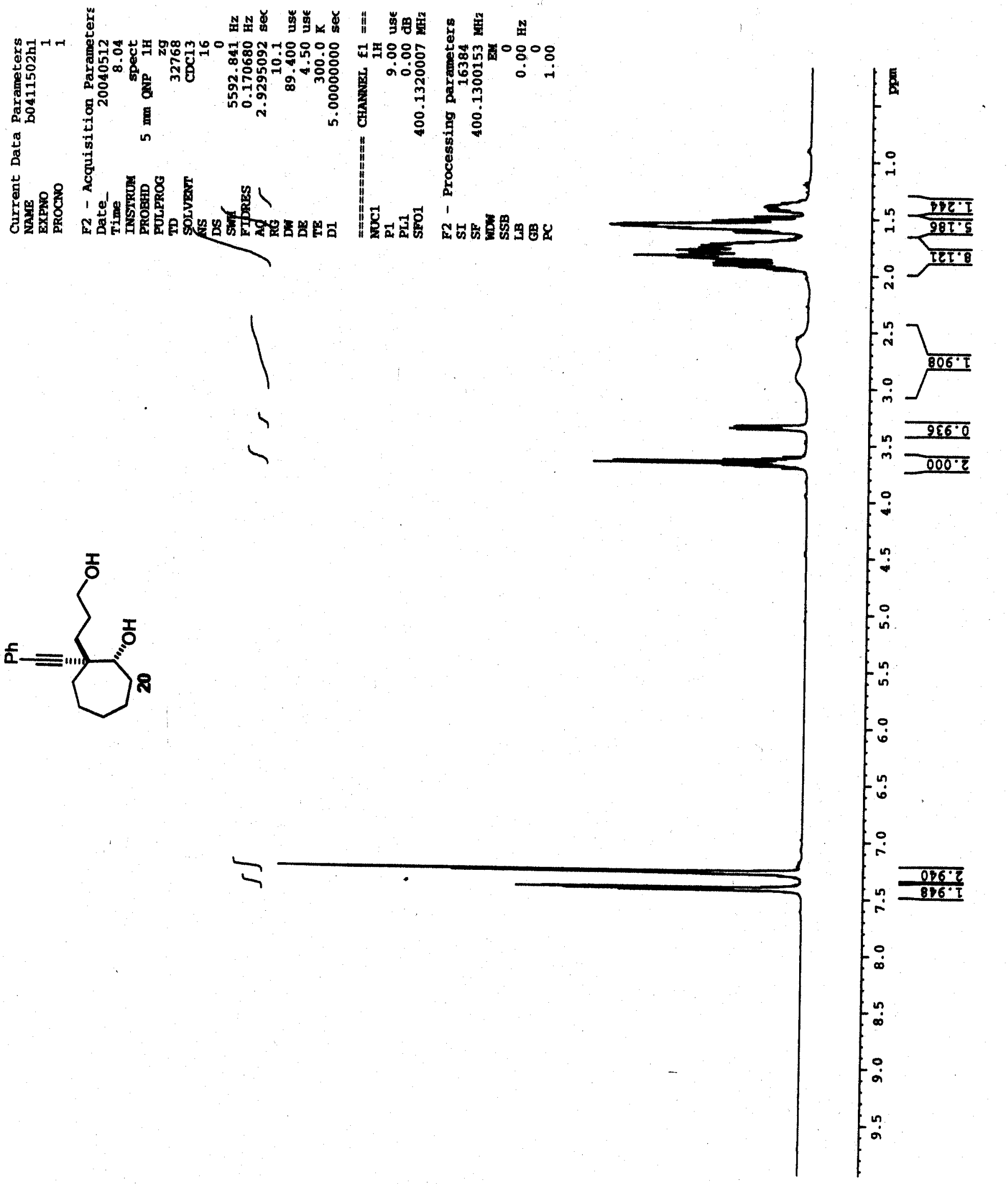

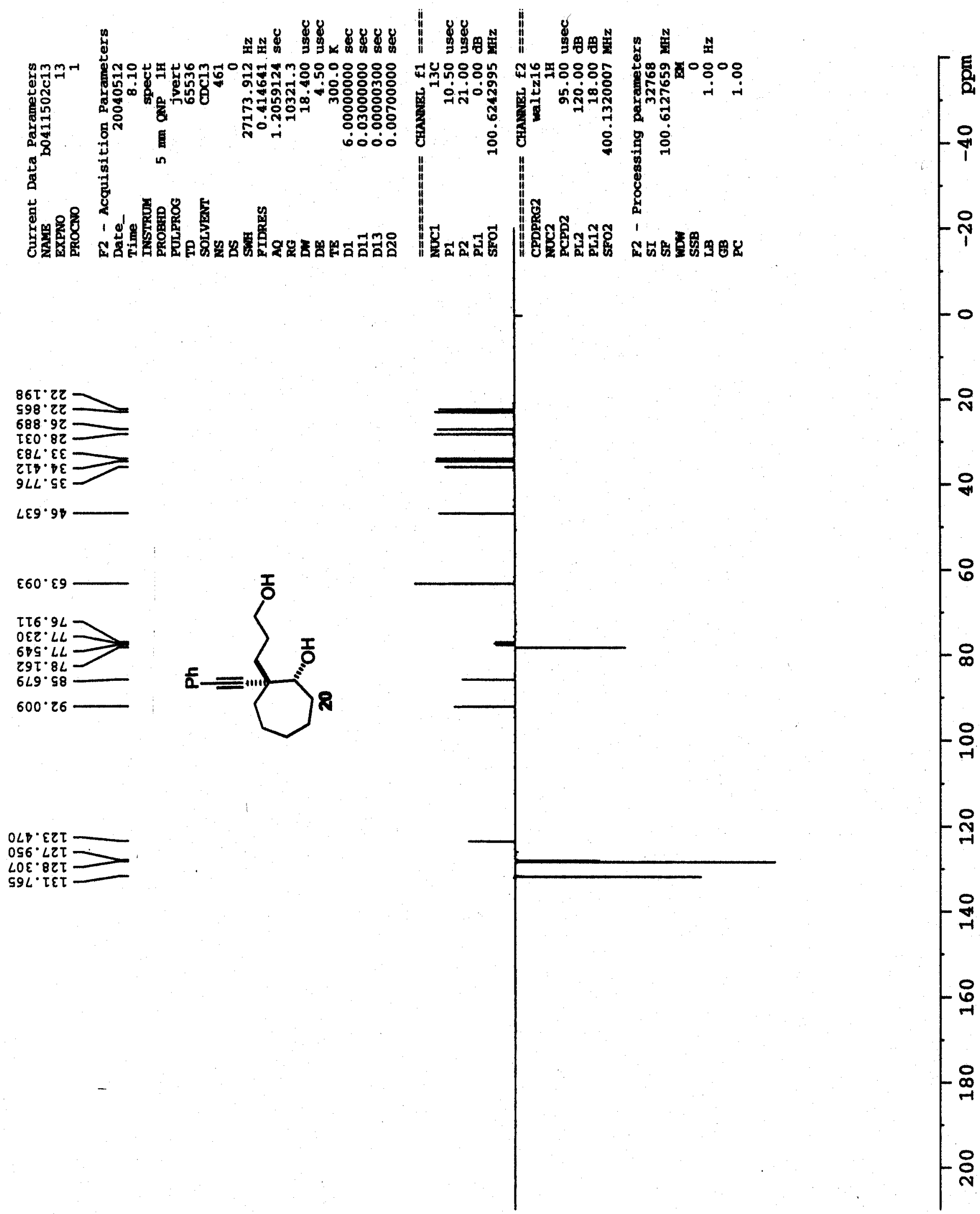

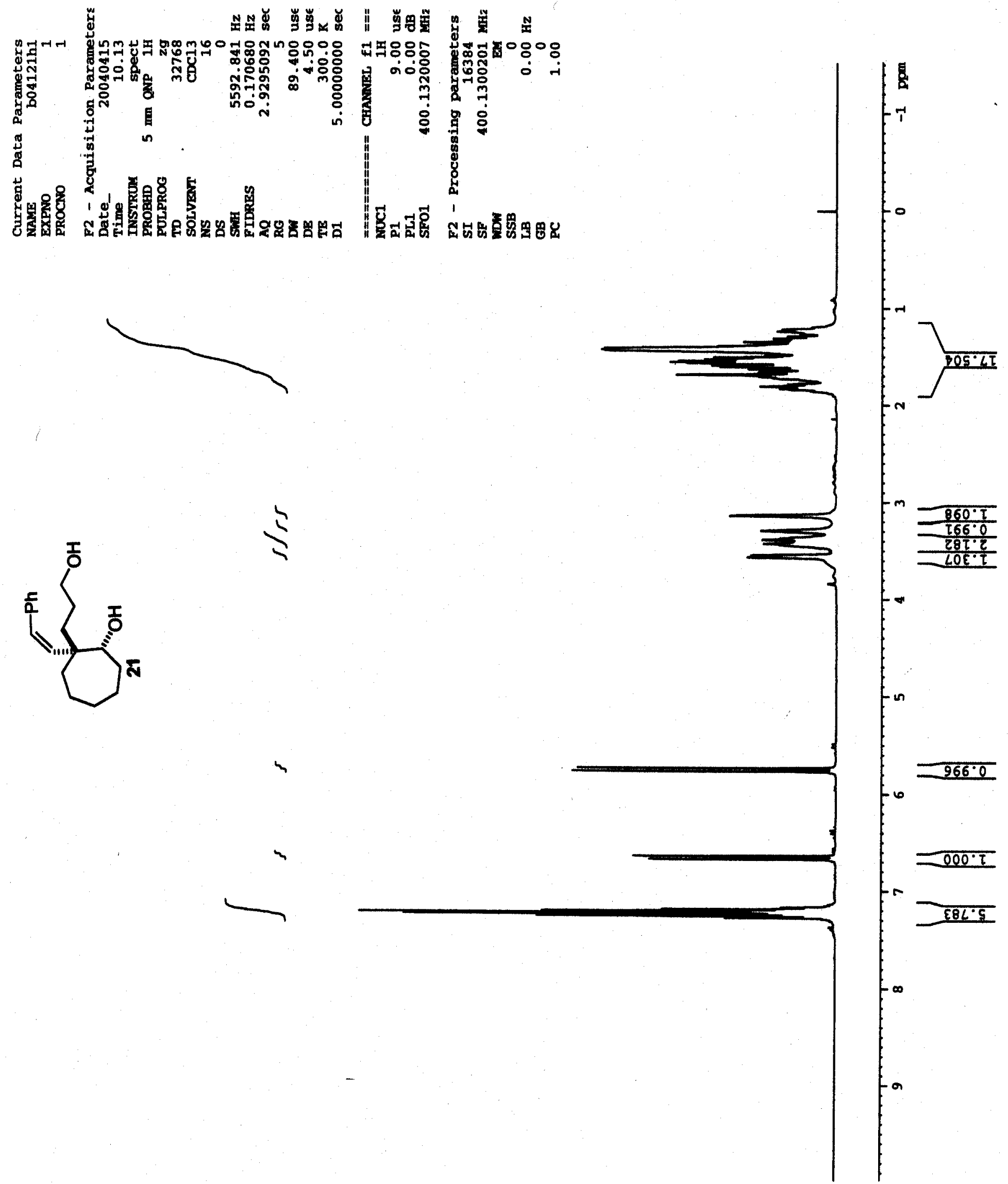

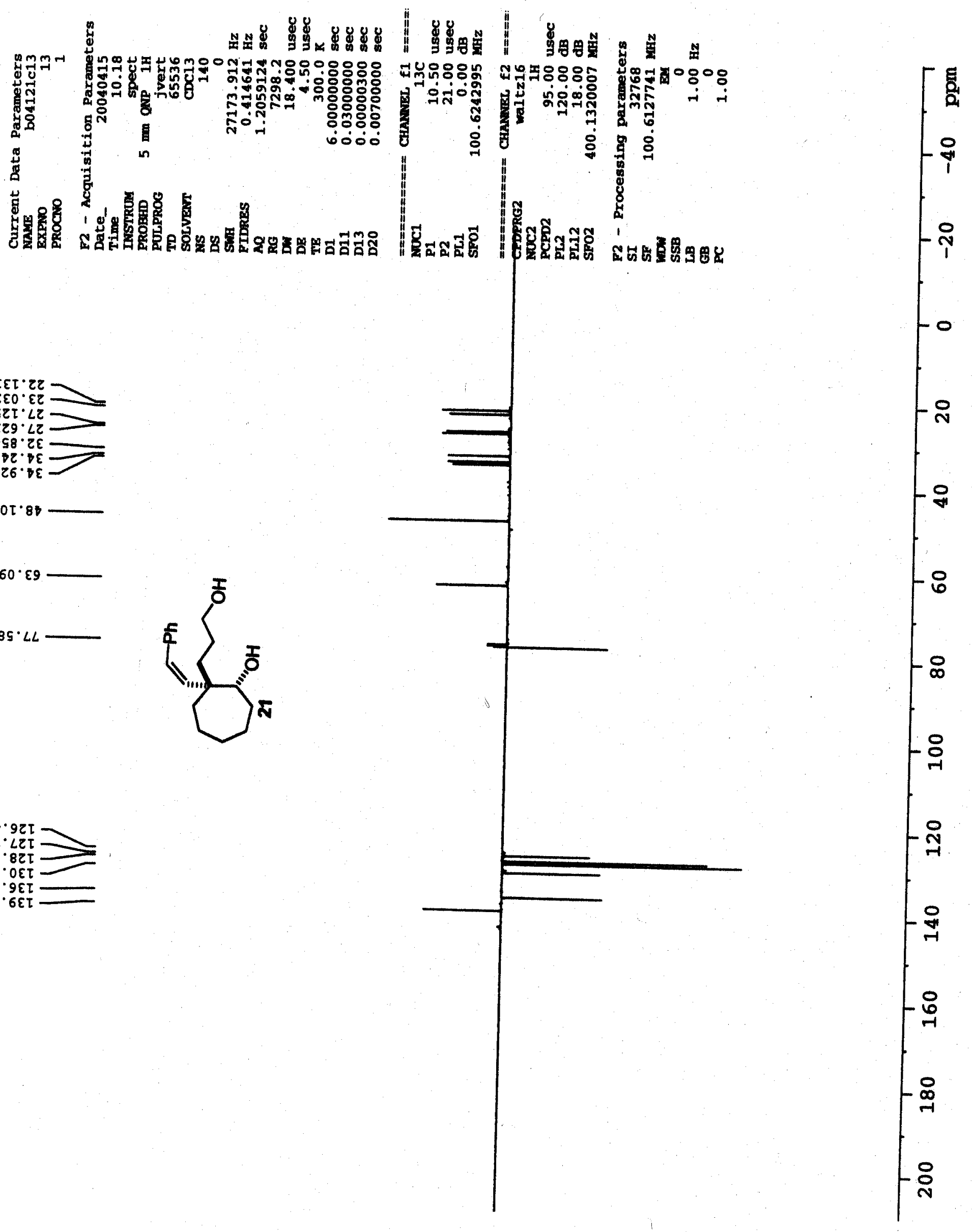

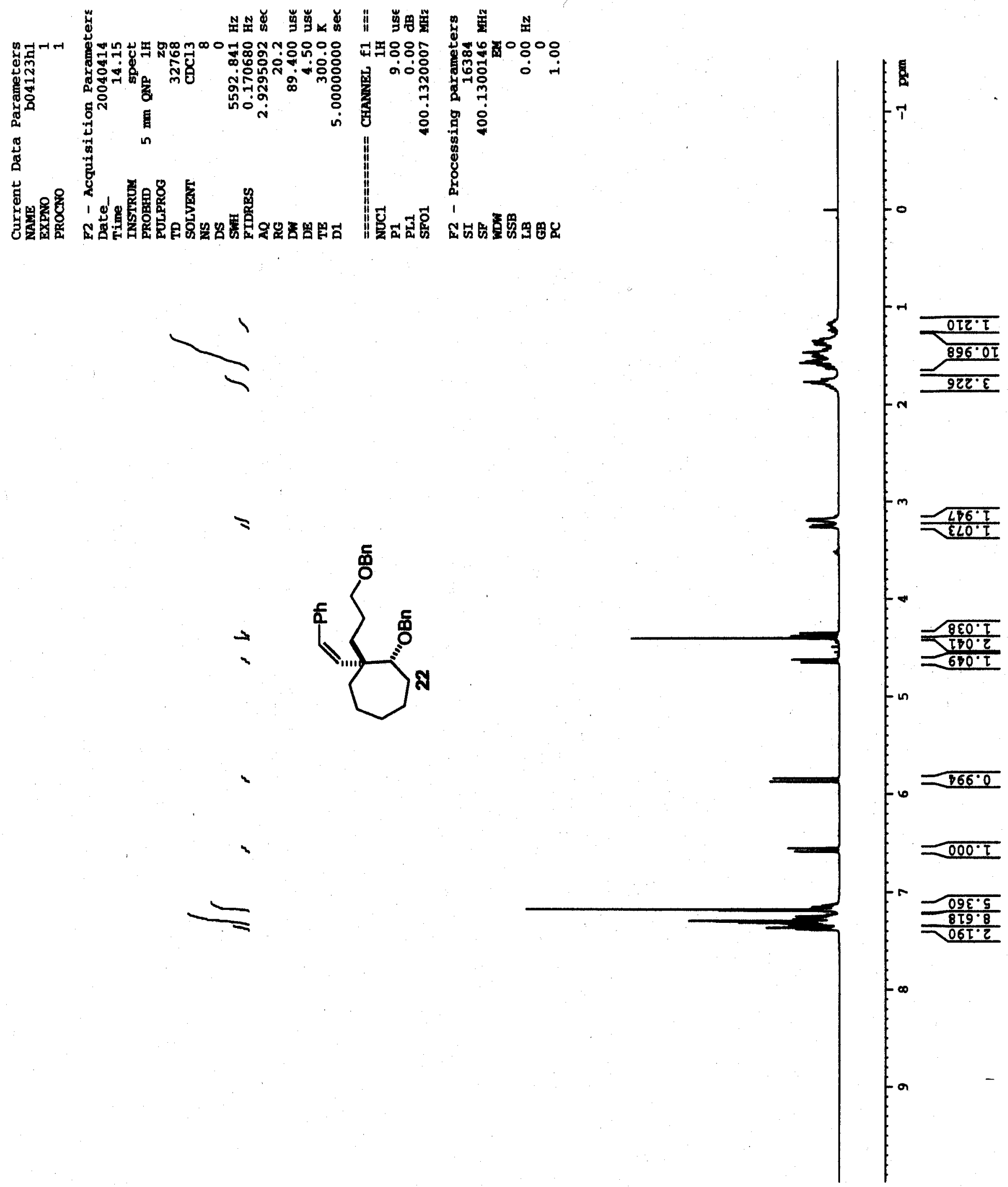

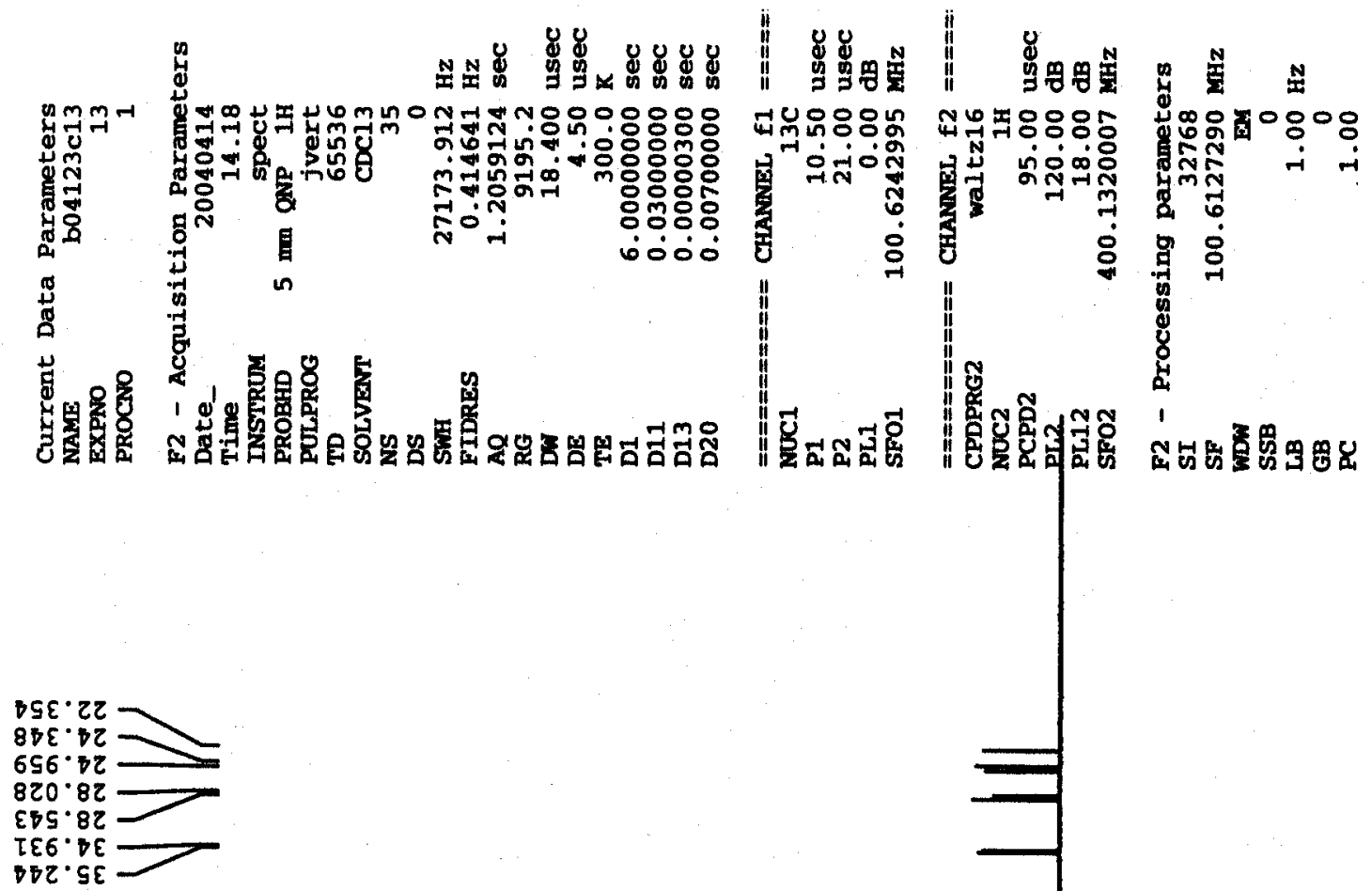

LOE $85 \square$
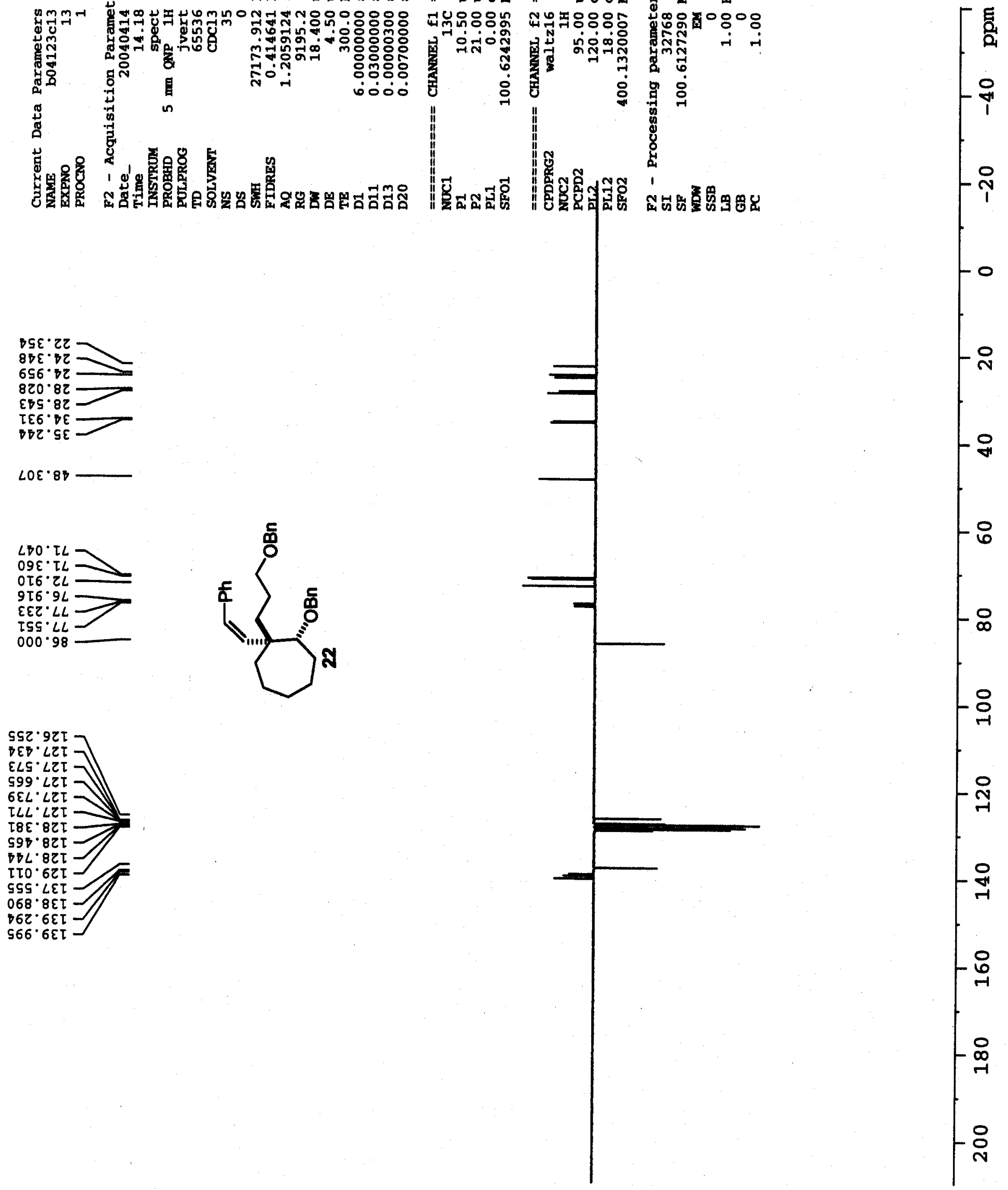

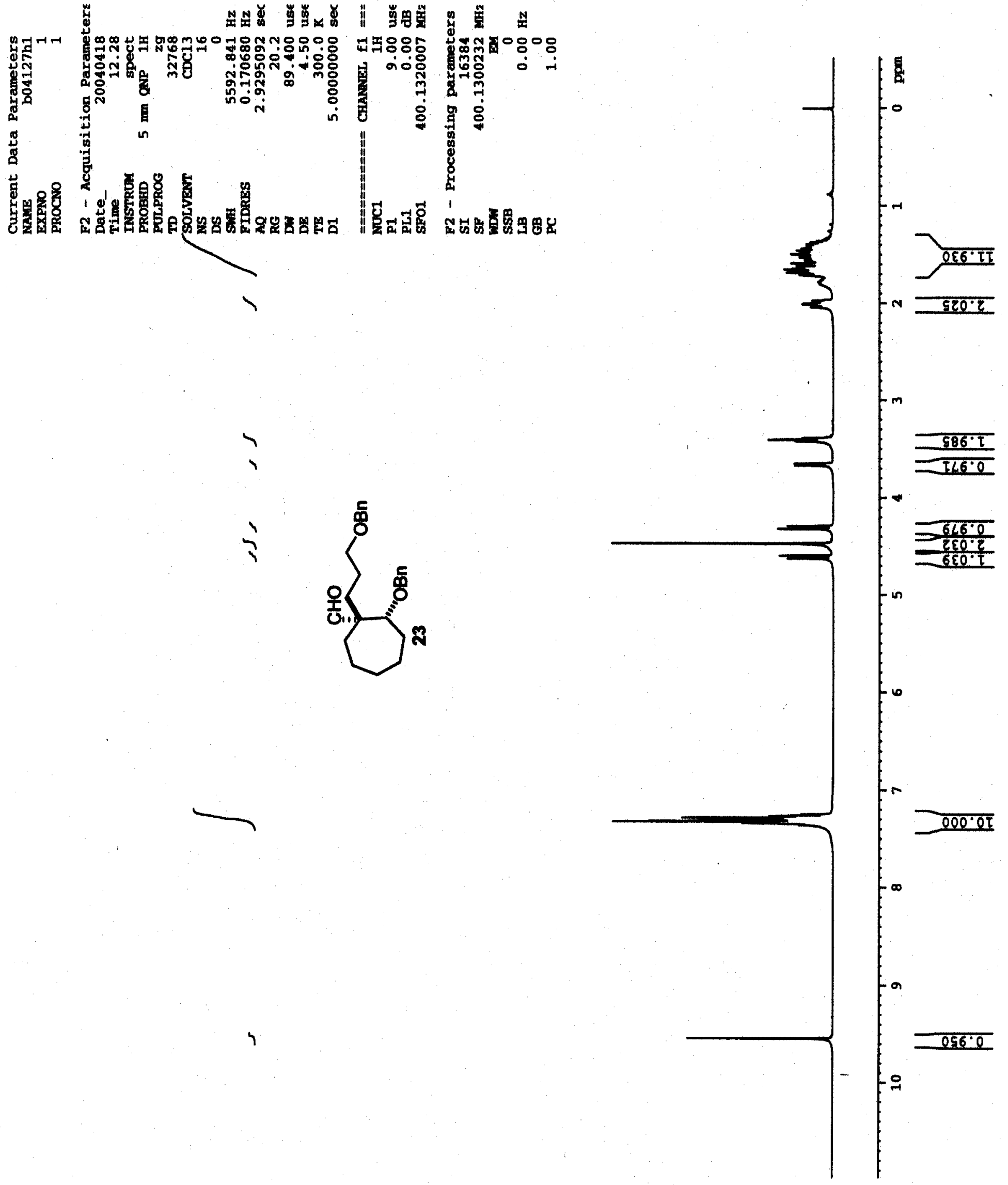

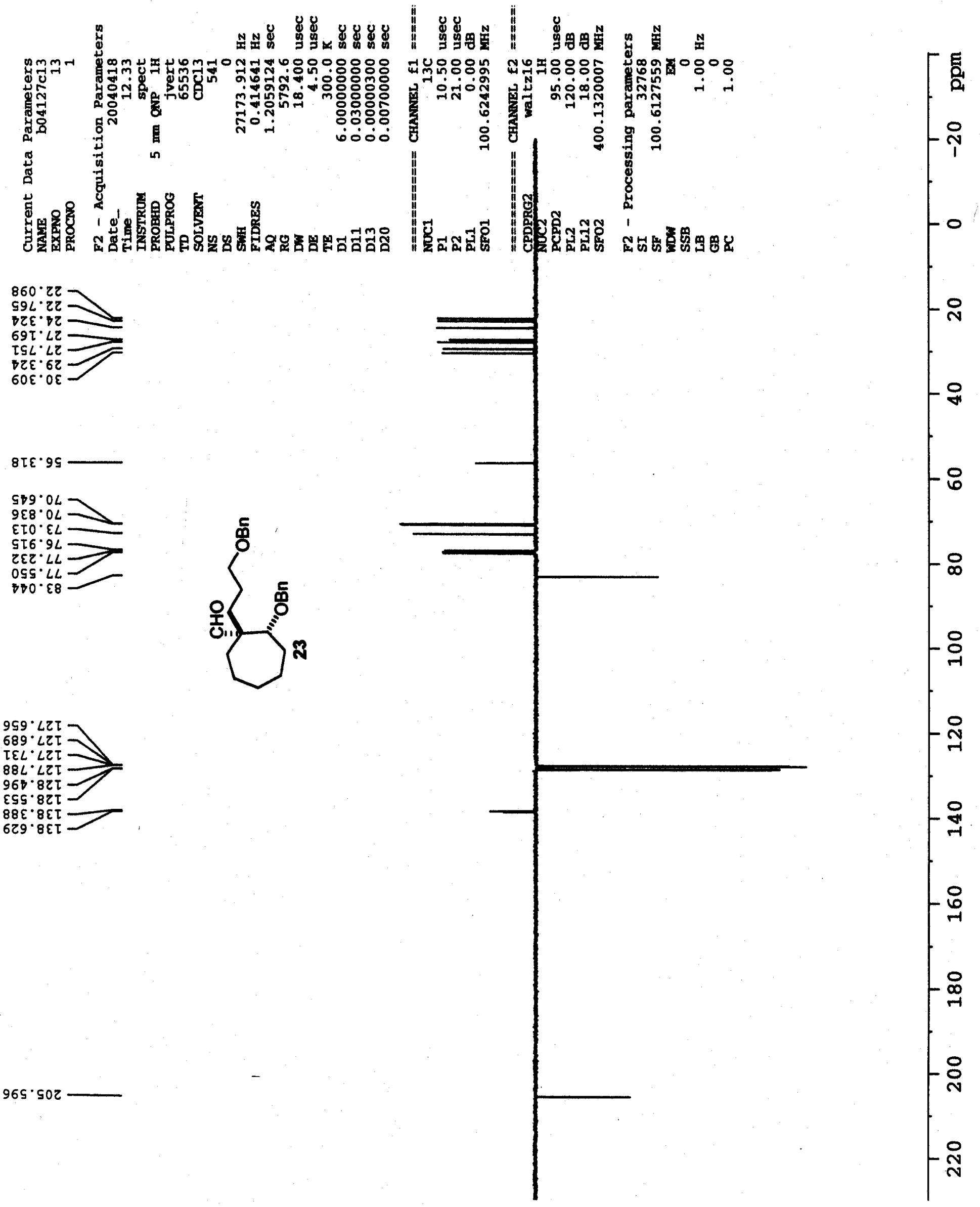

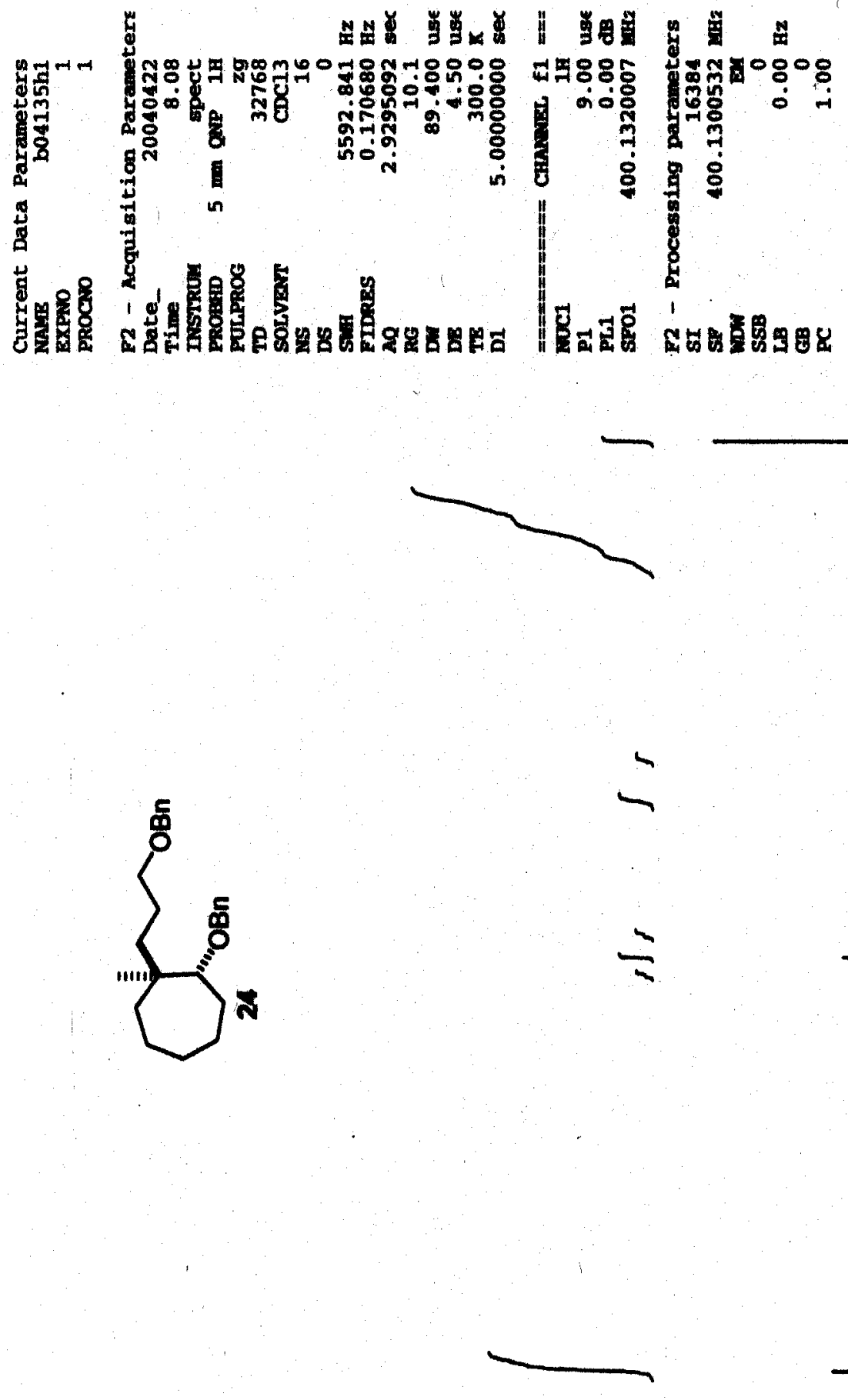

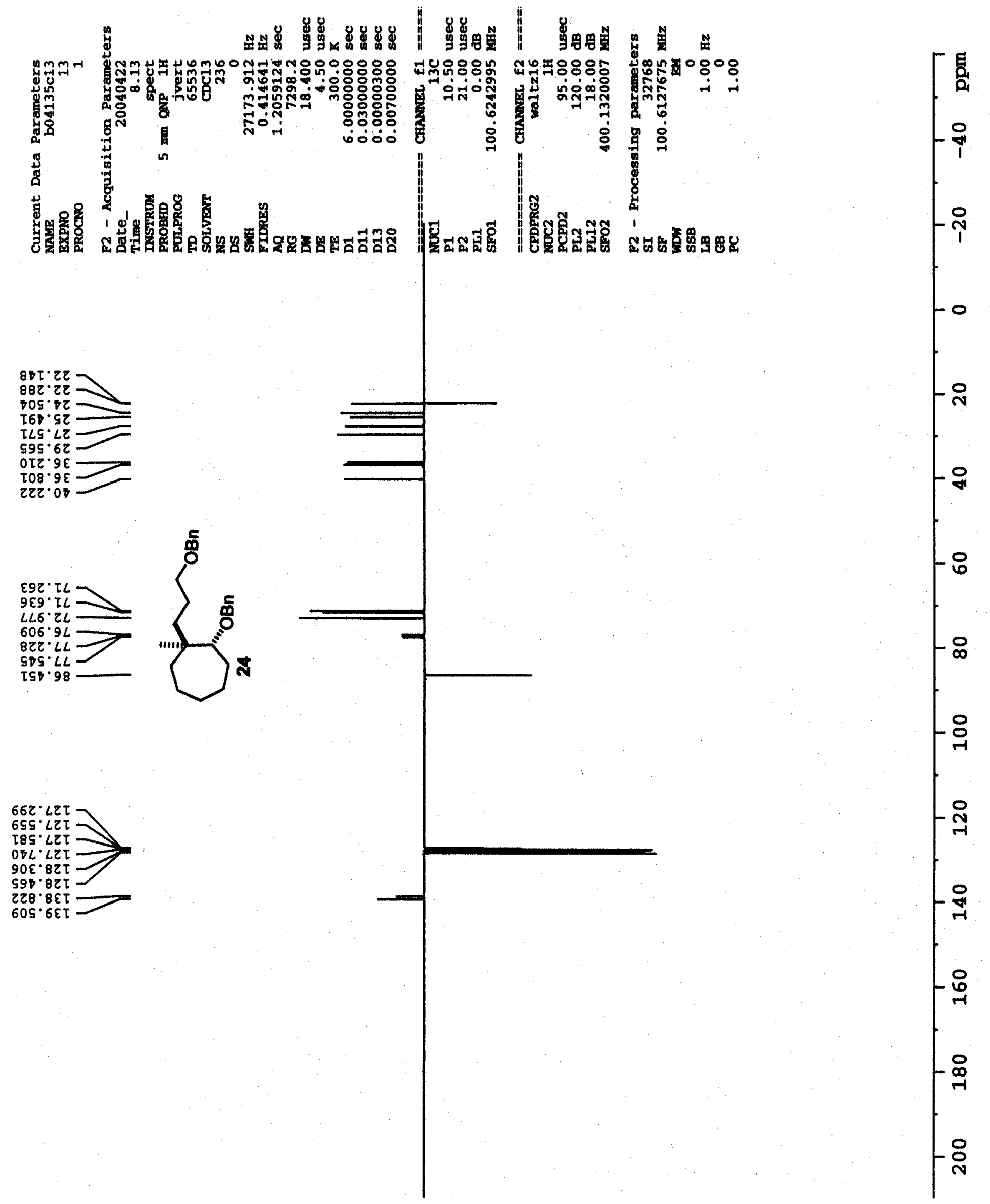

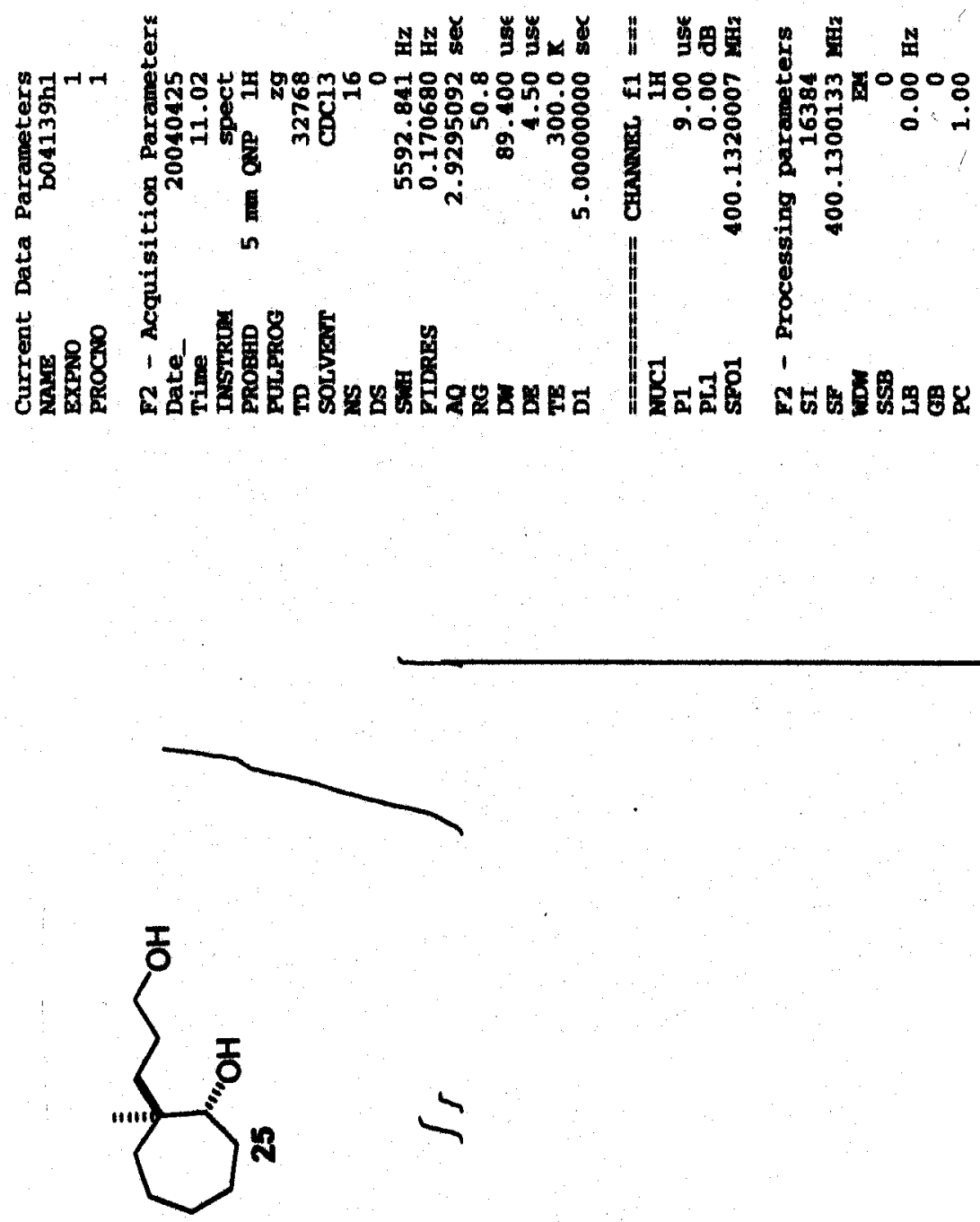

5

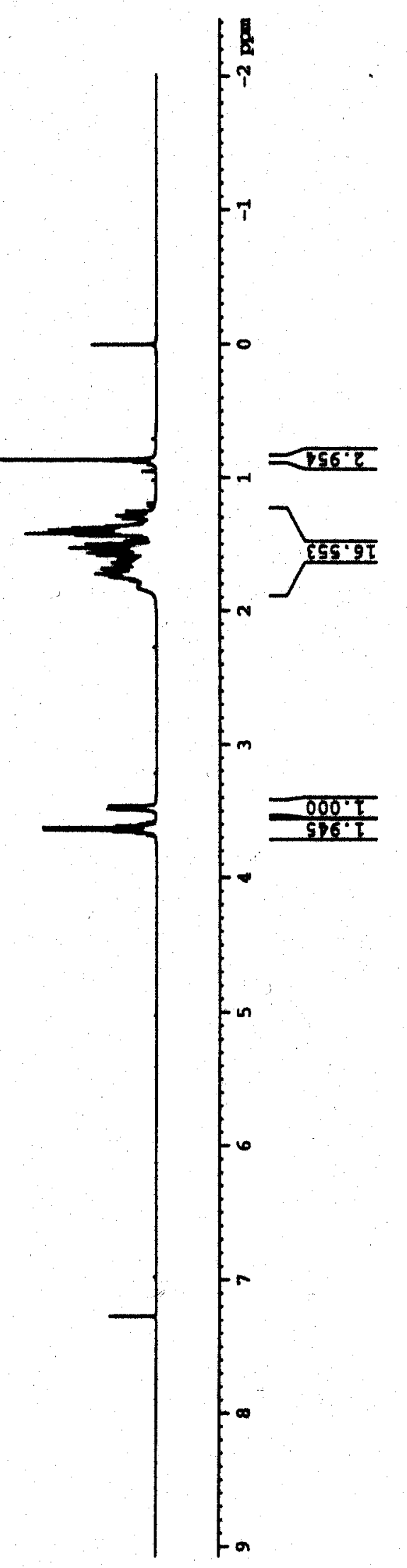



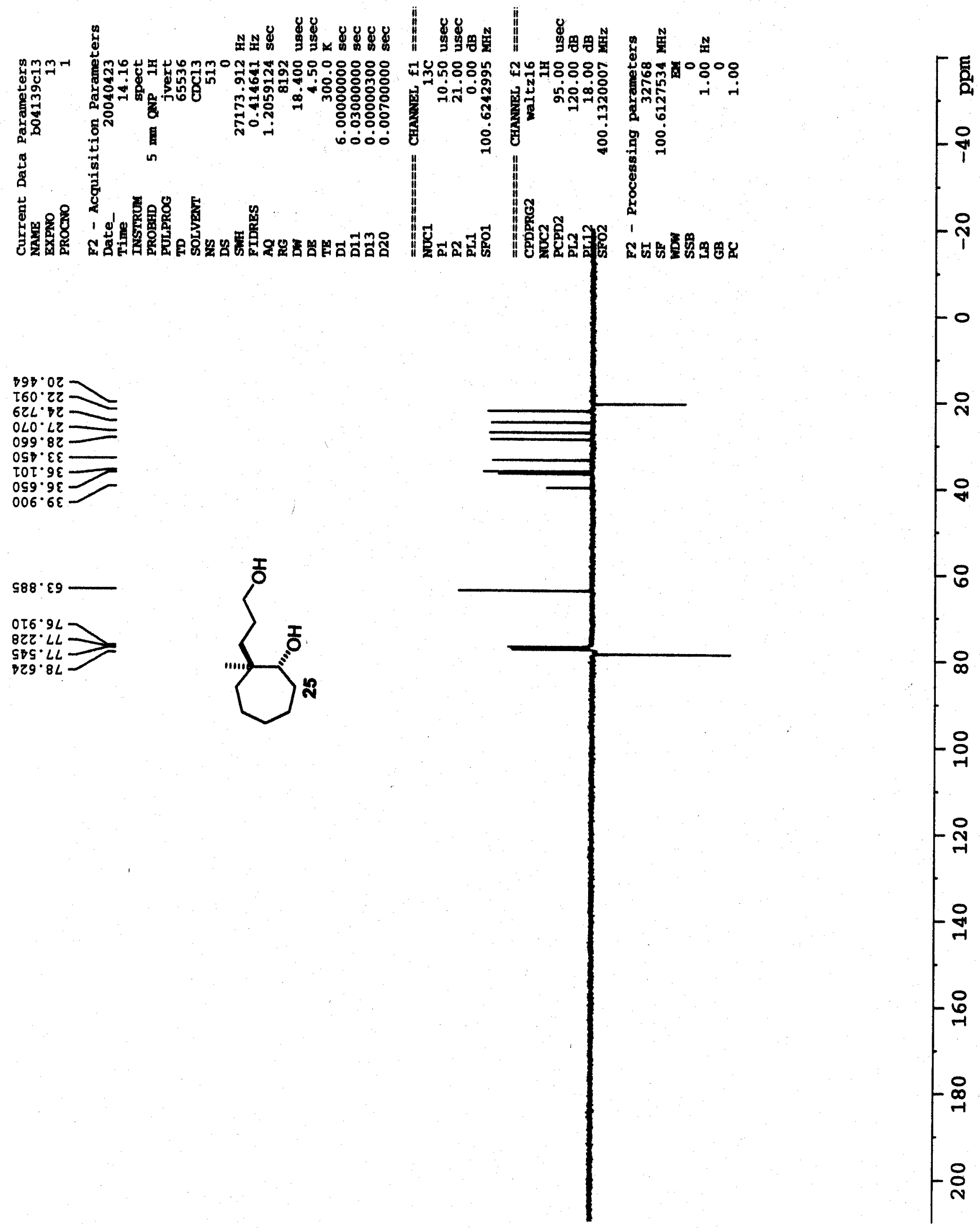


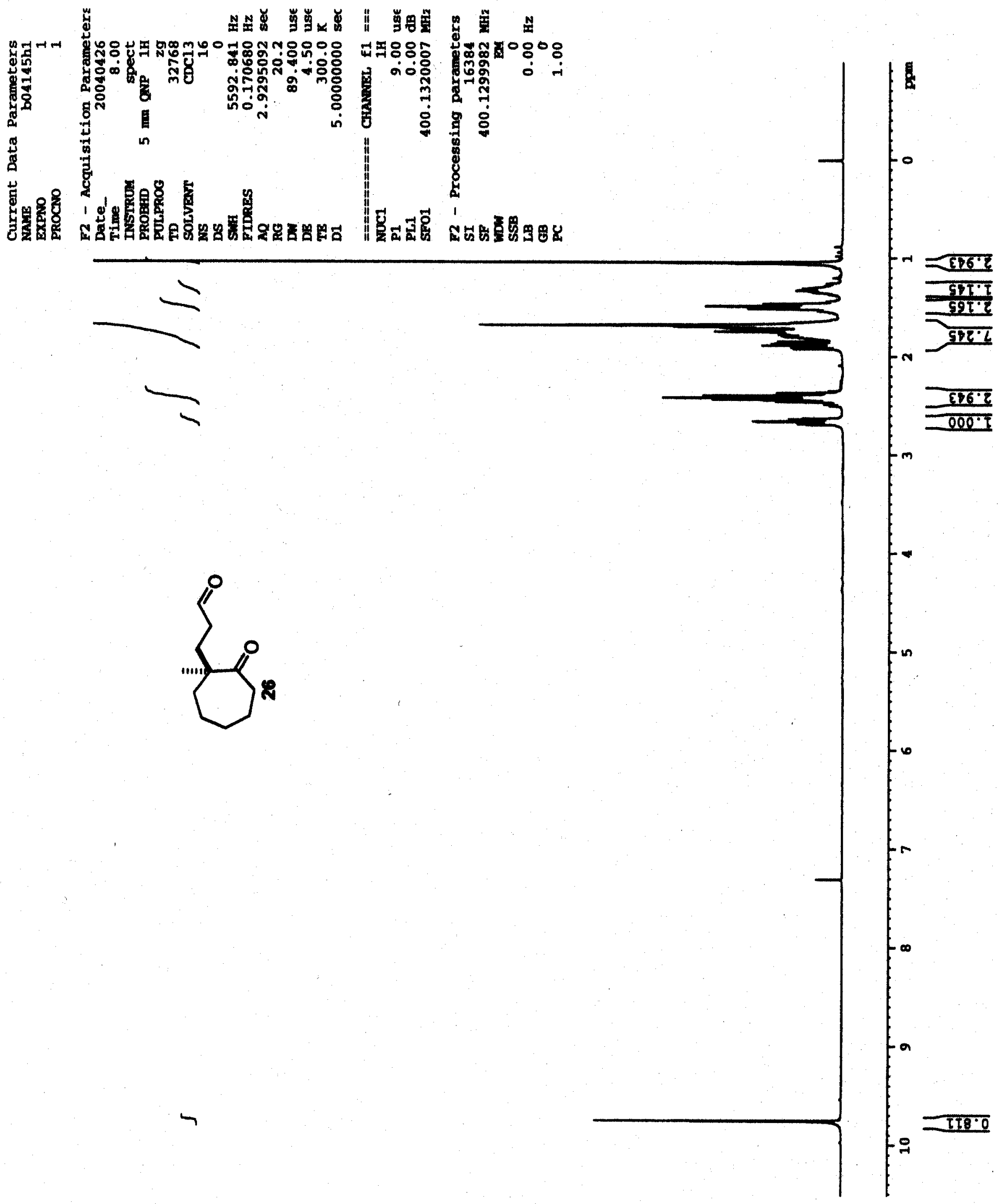



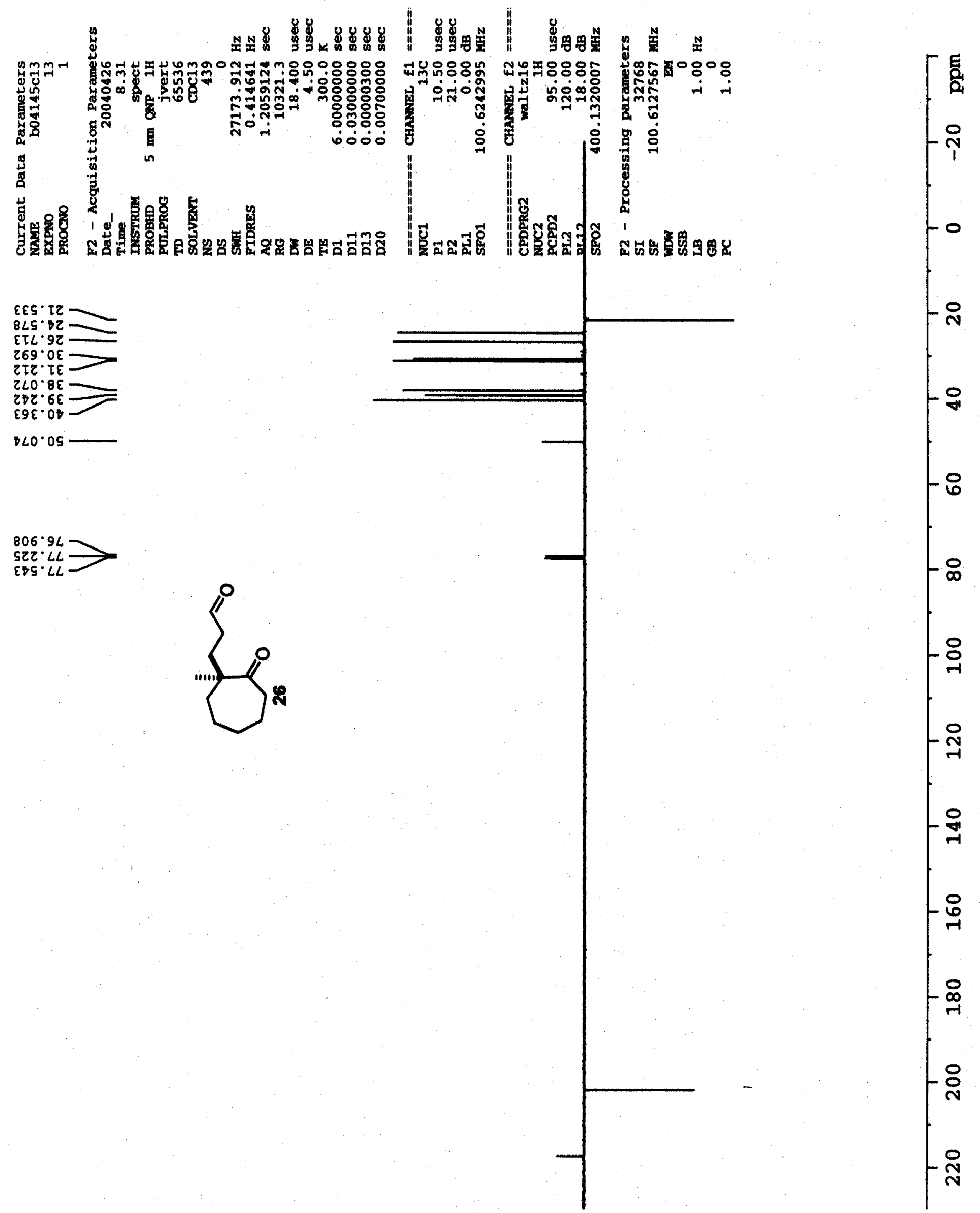

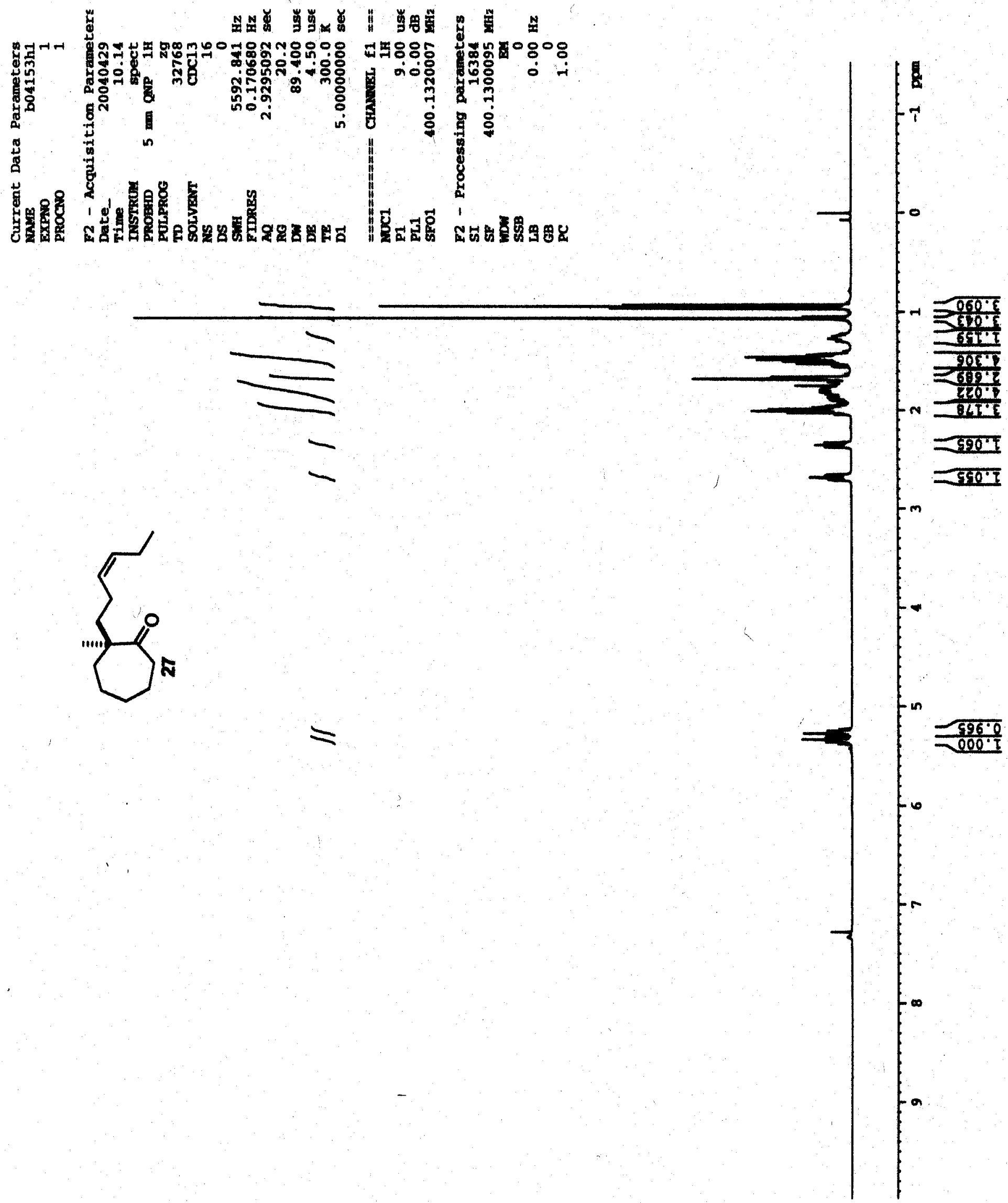


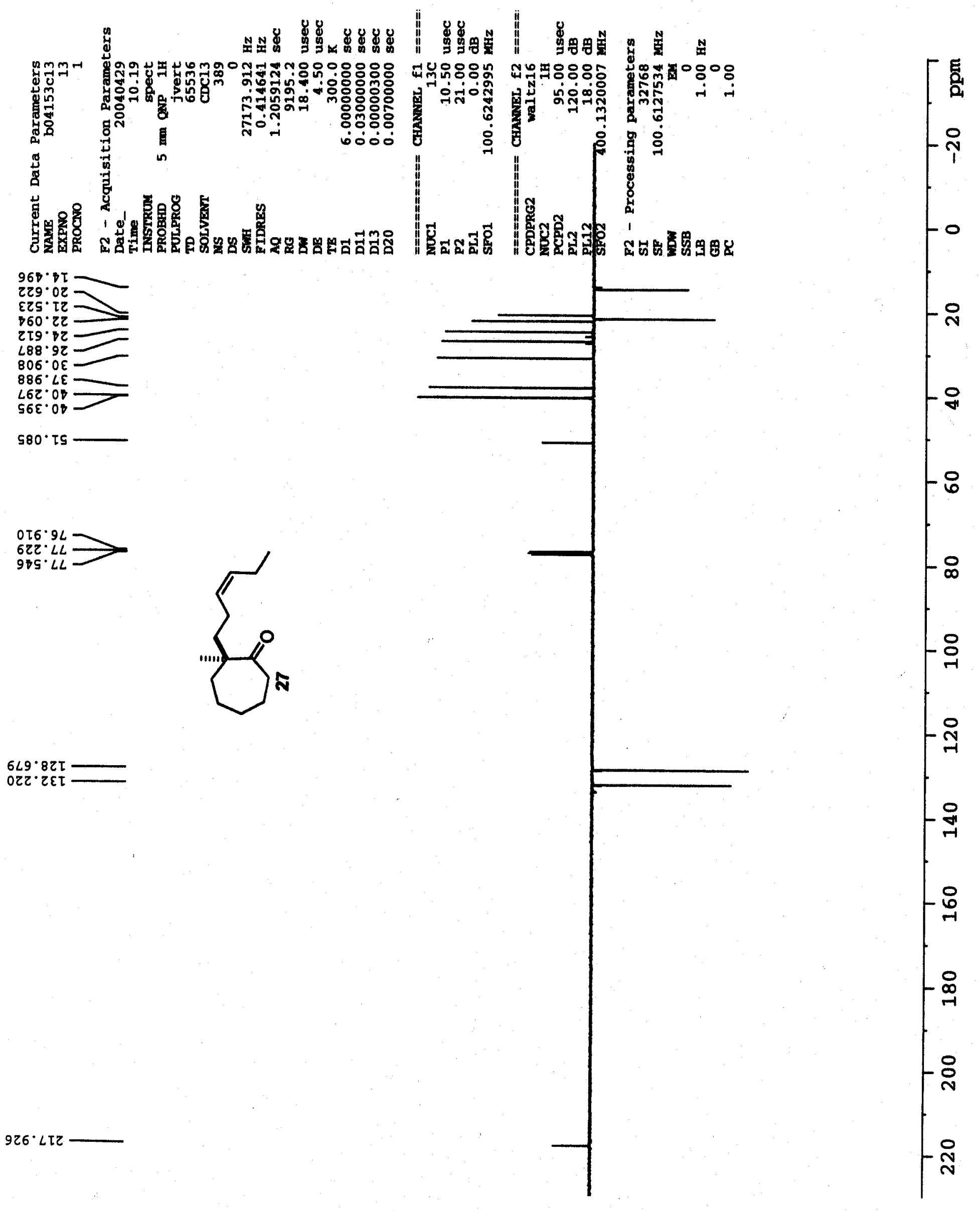



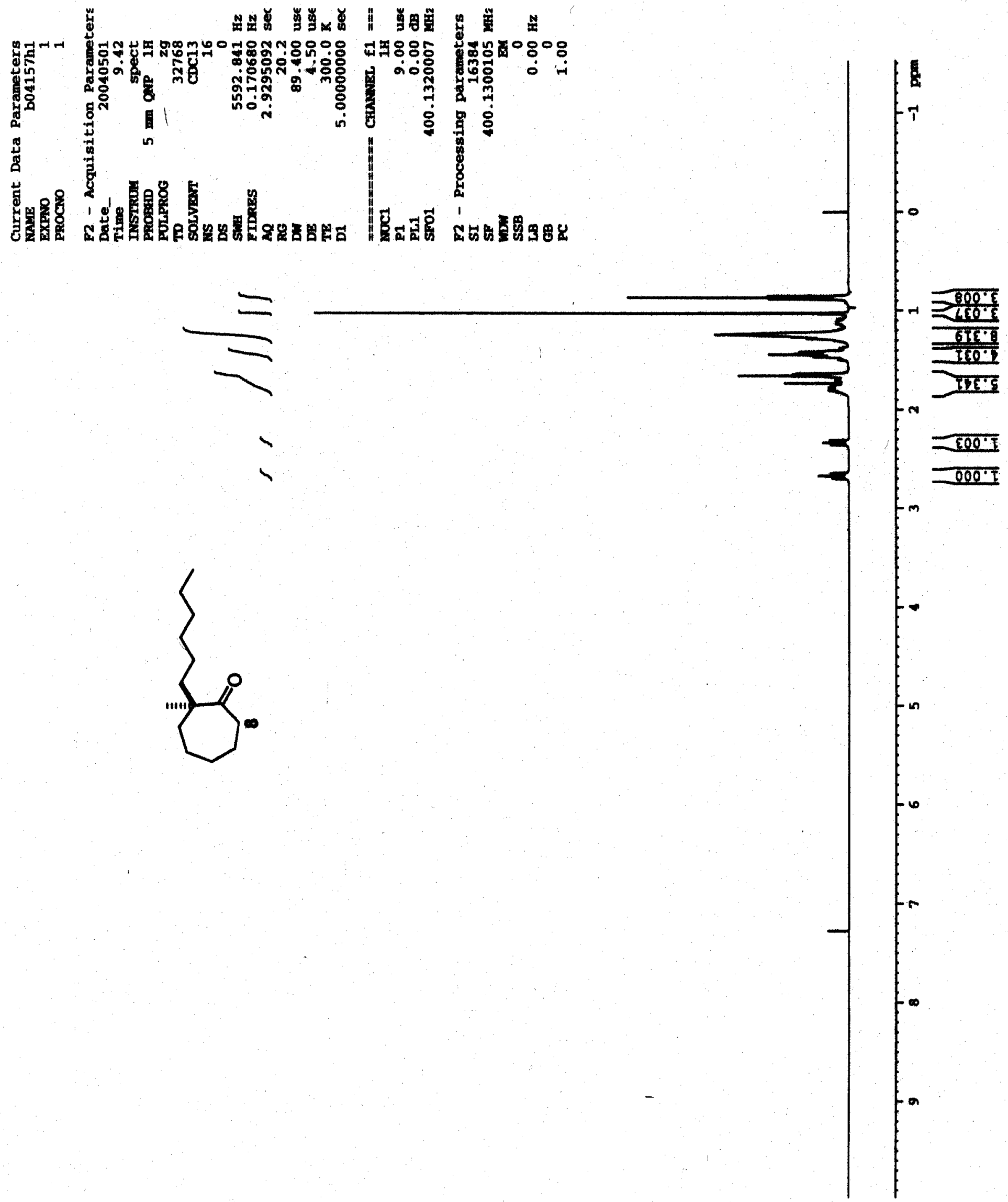


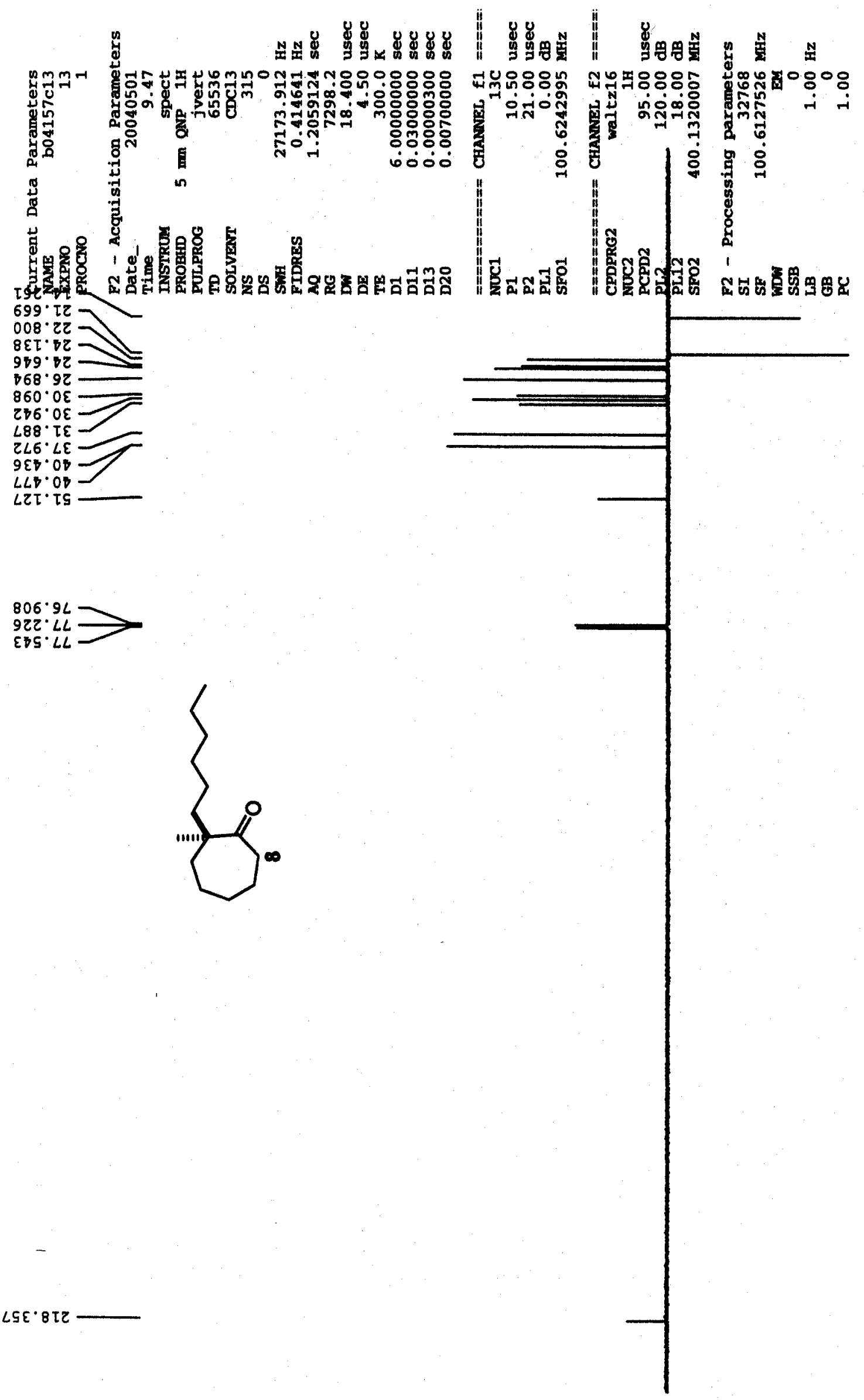

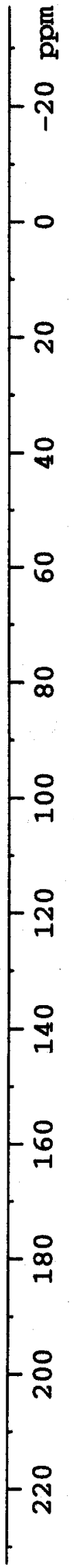



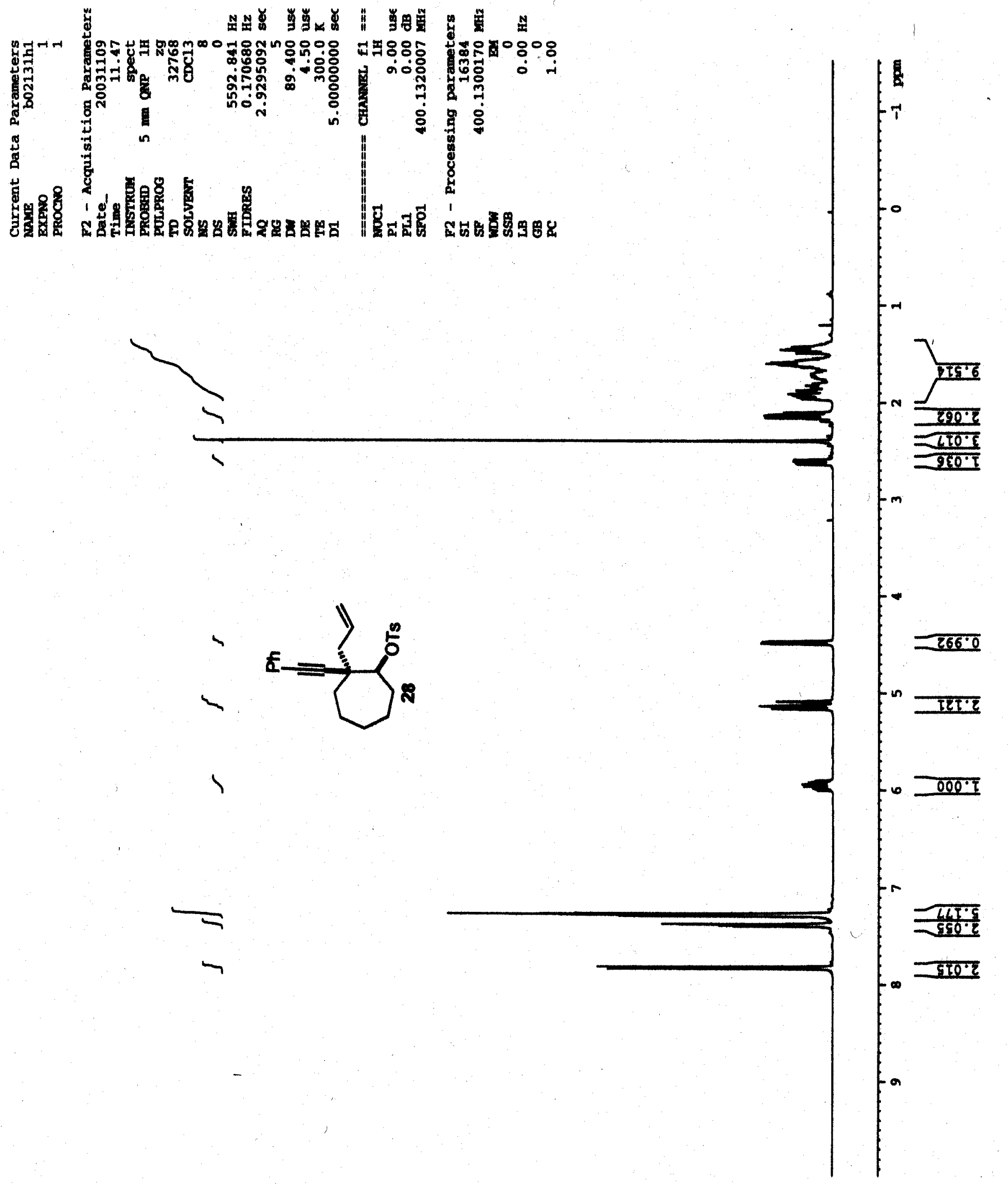

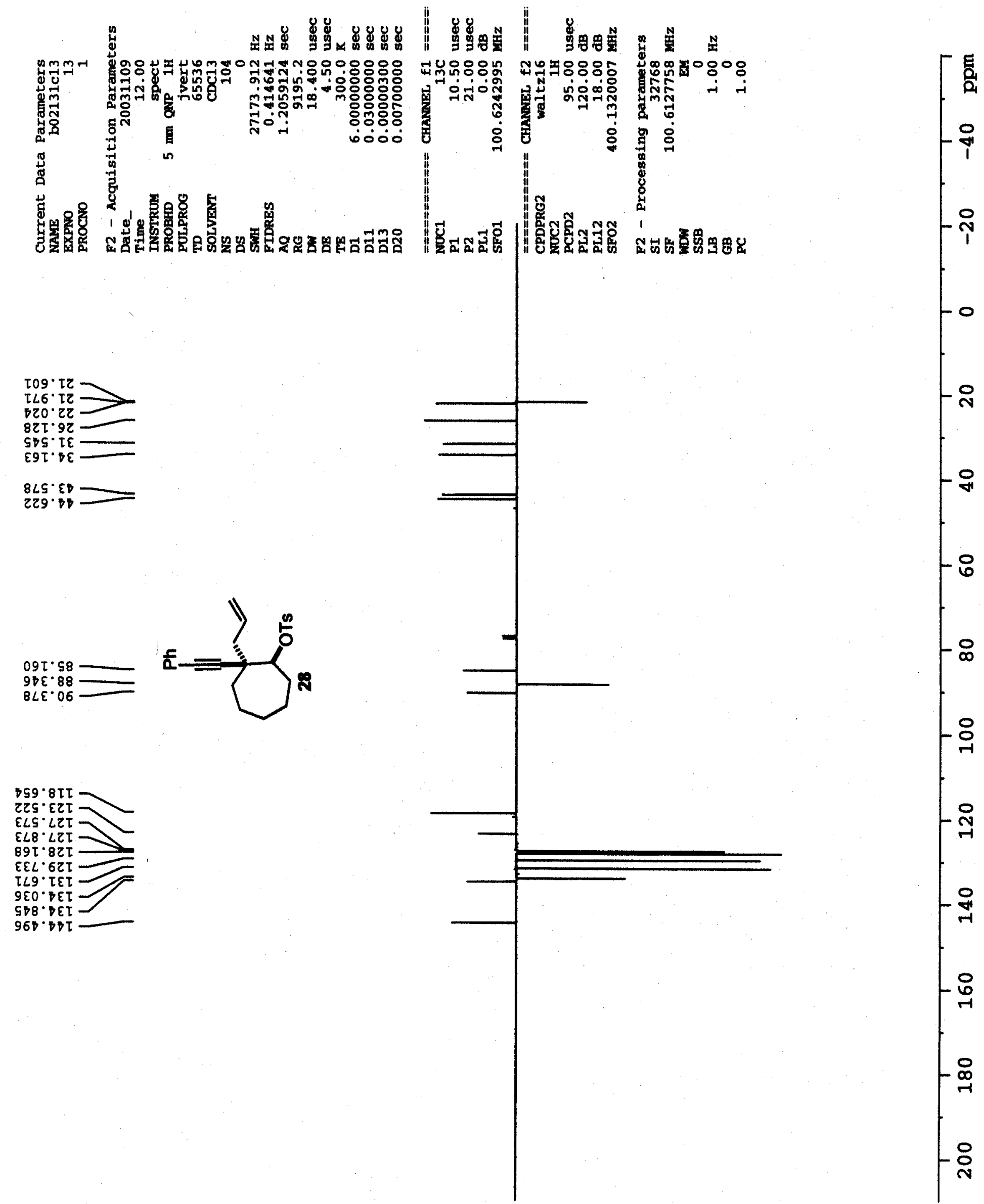

오

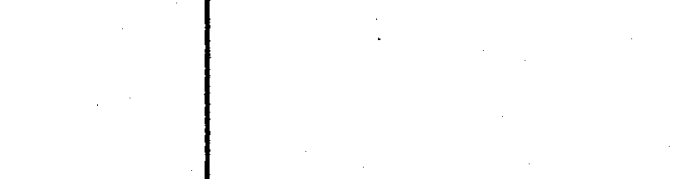



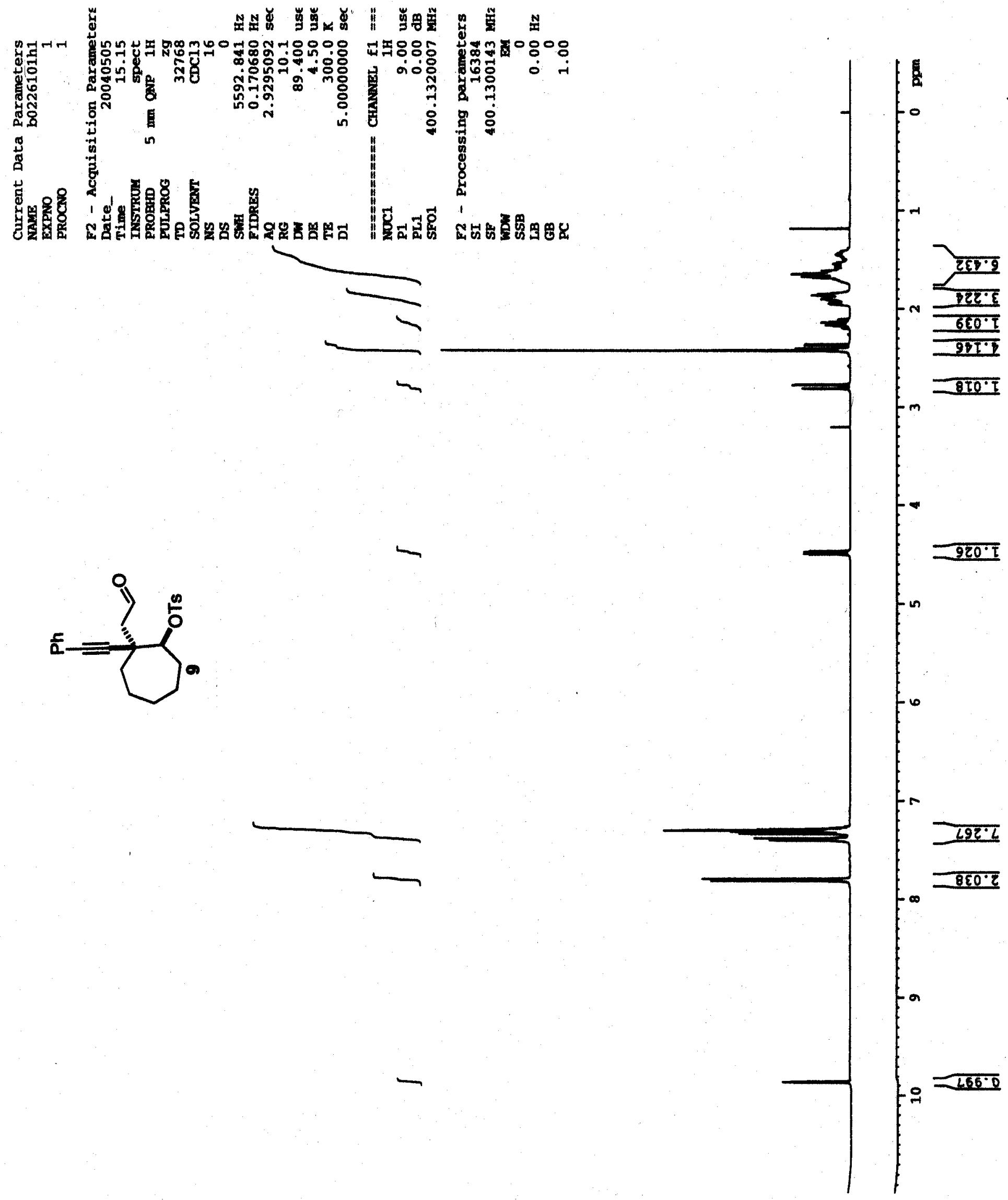

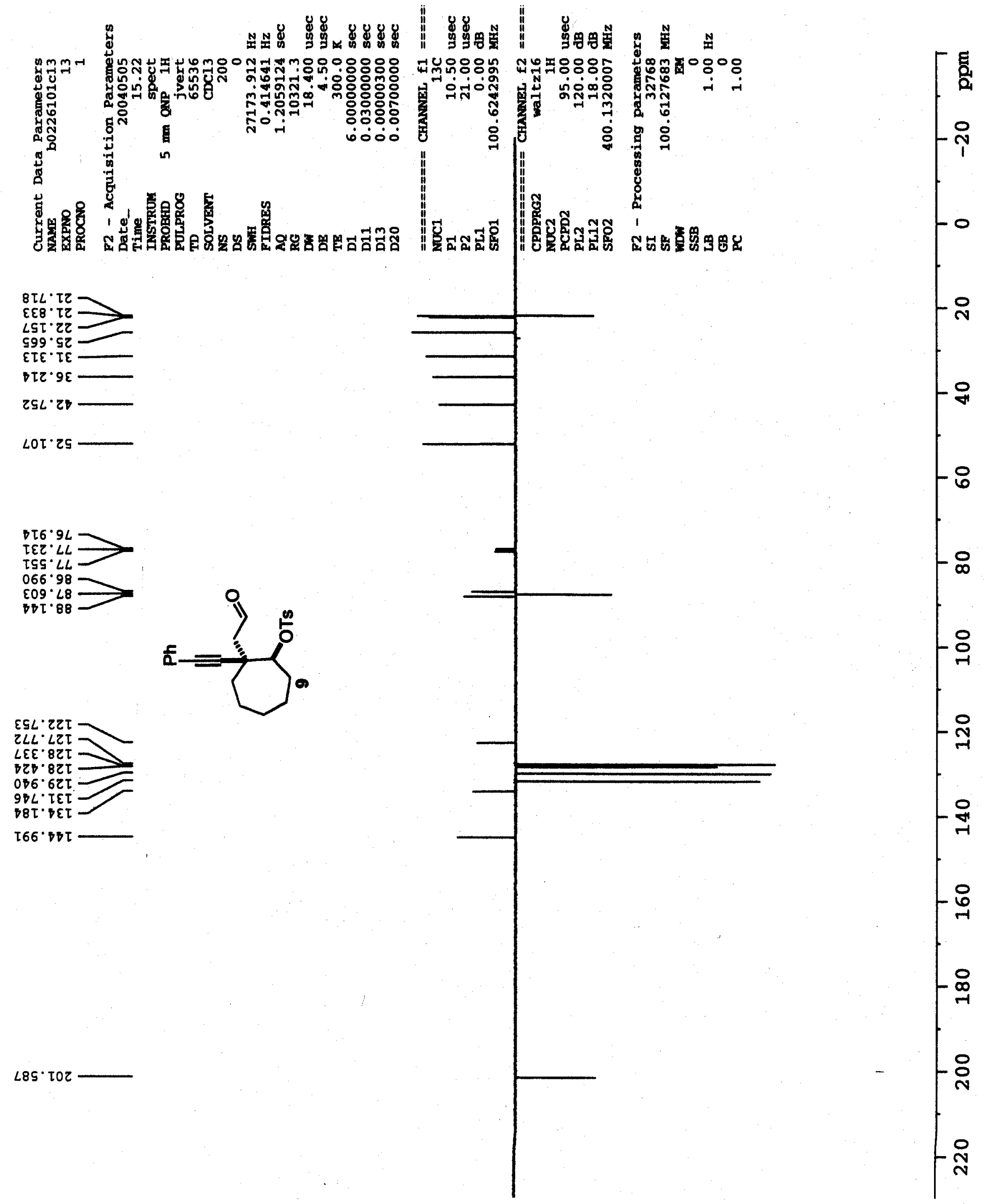

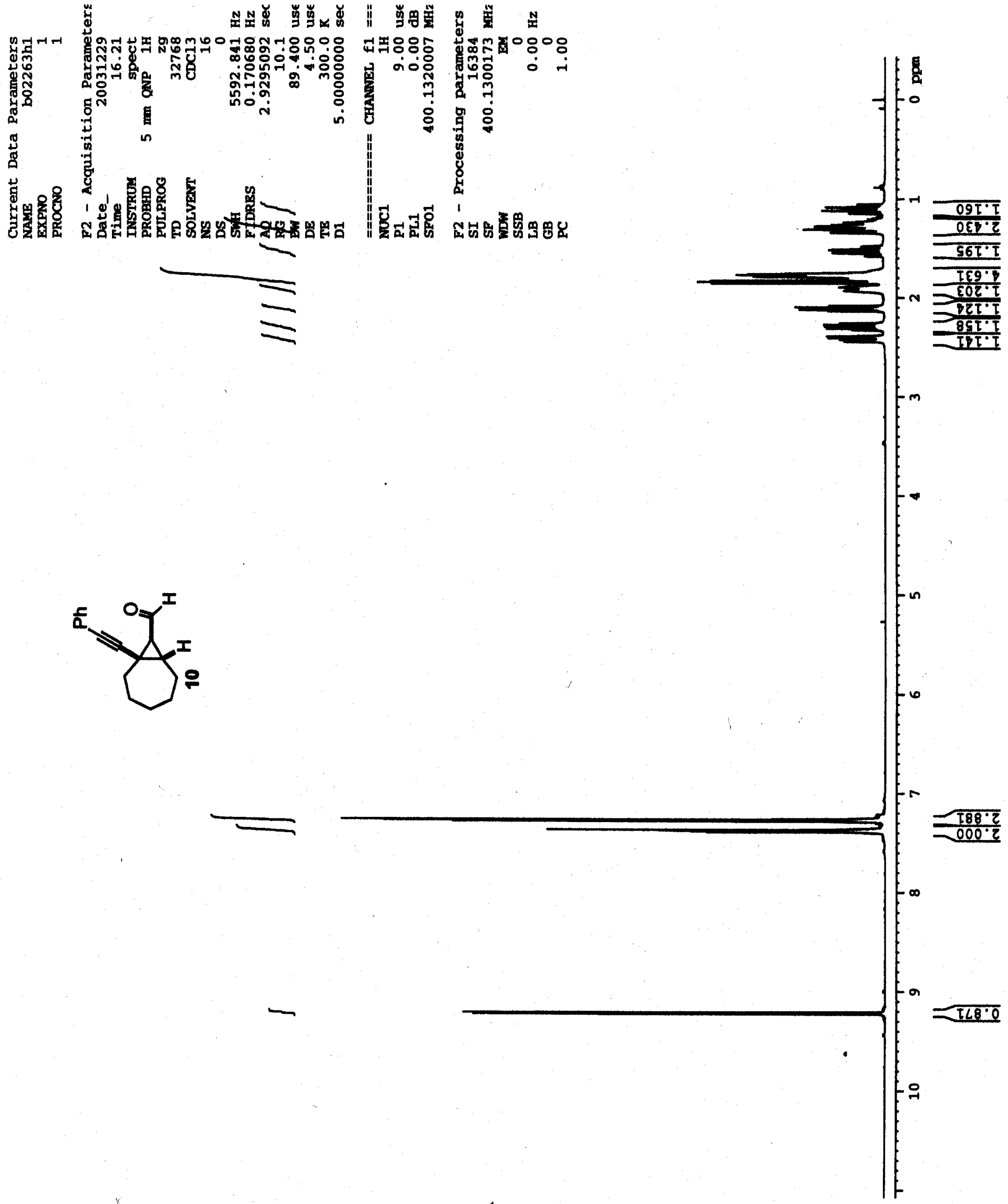

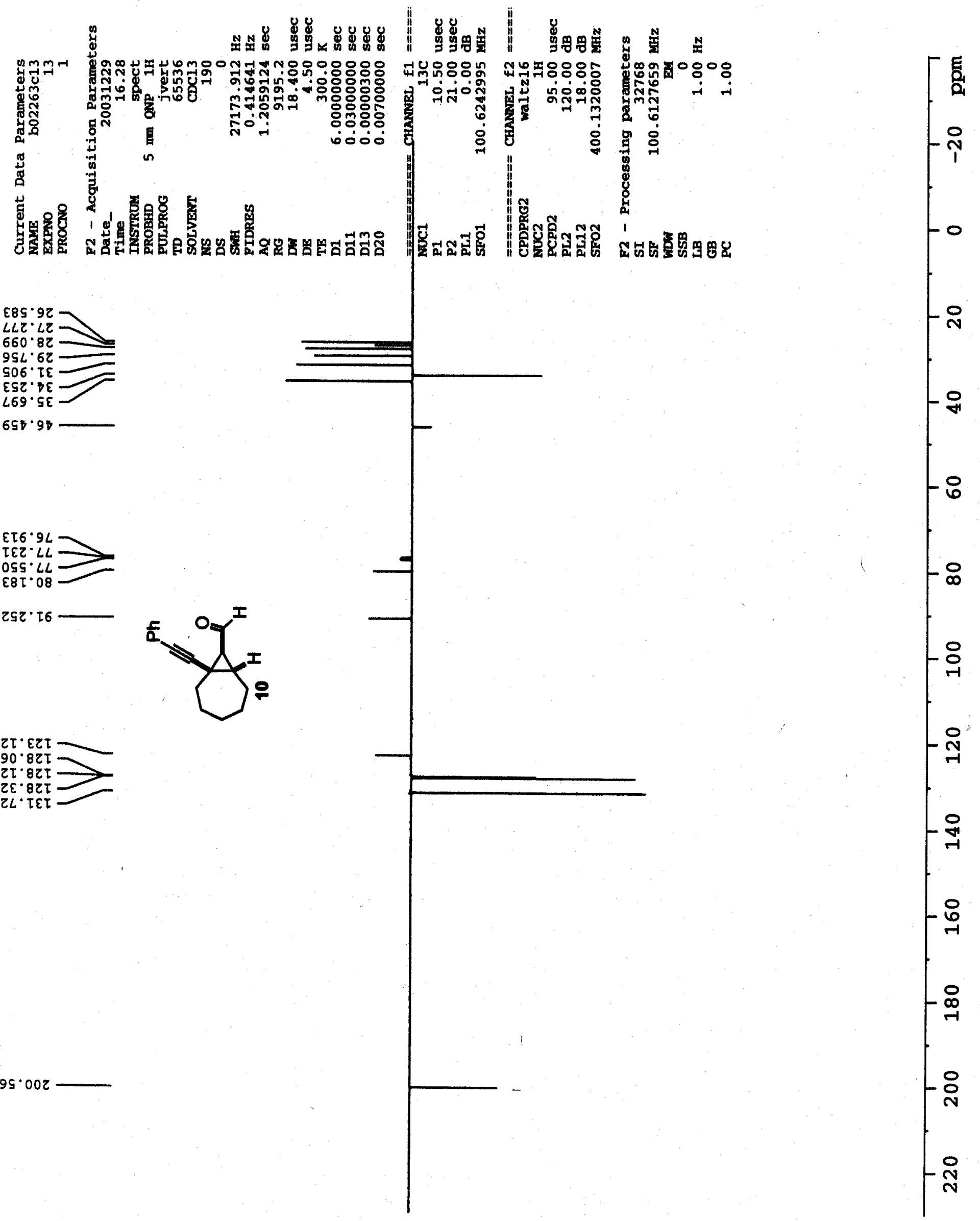

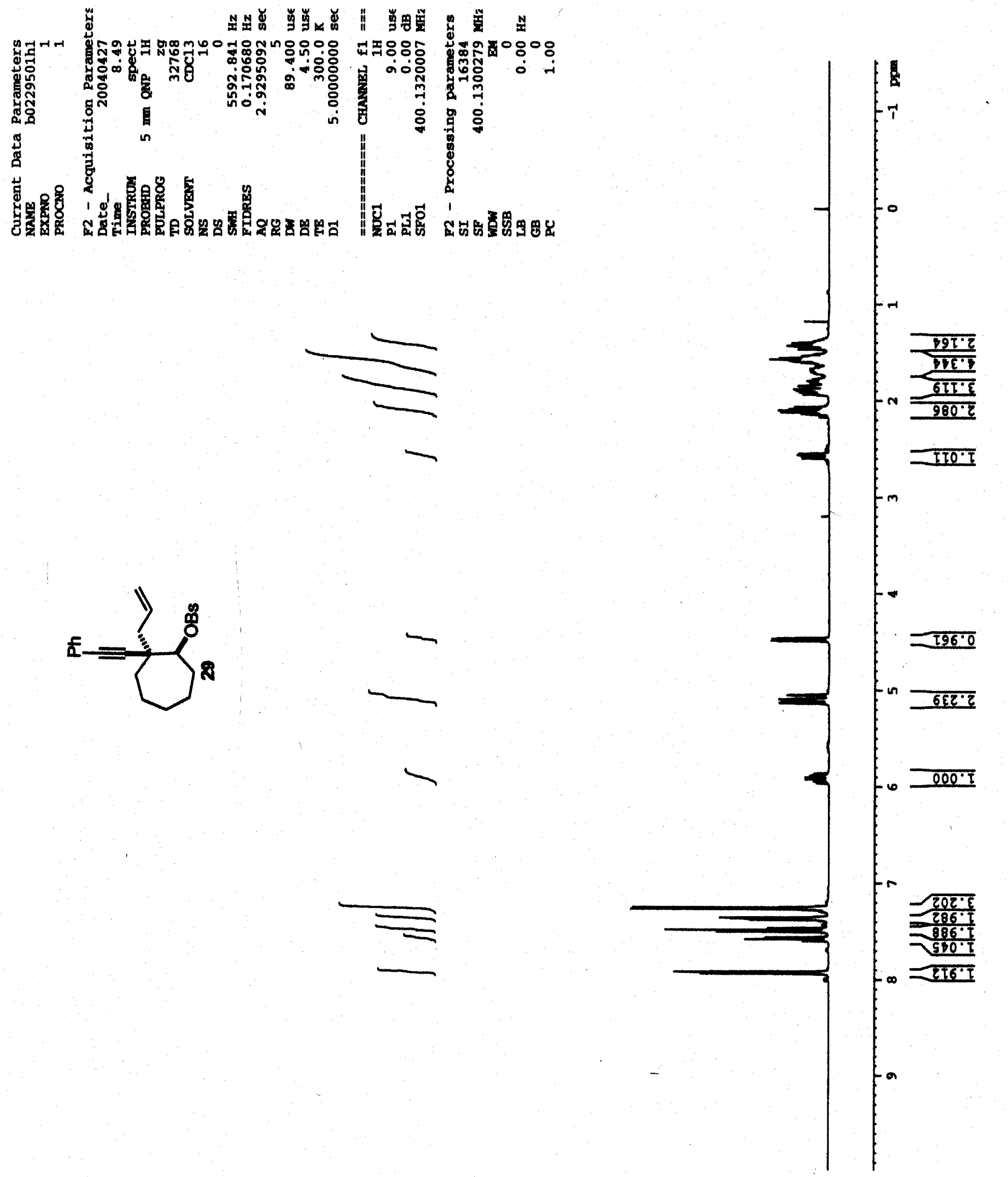

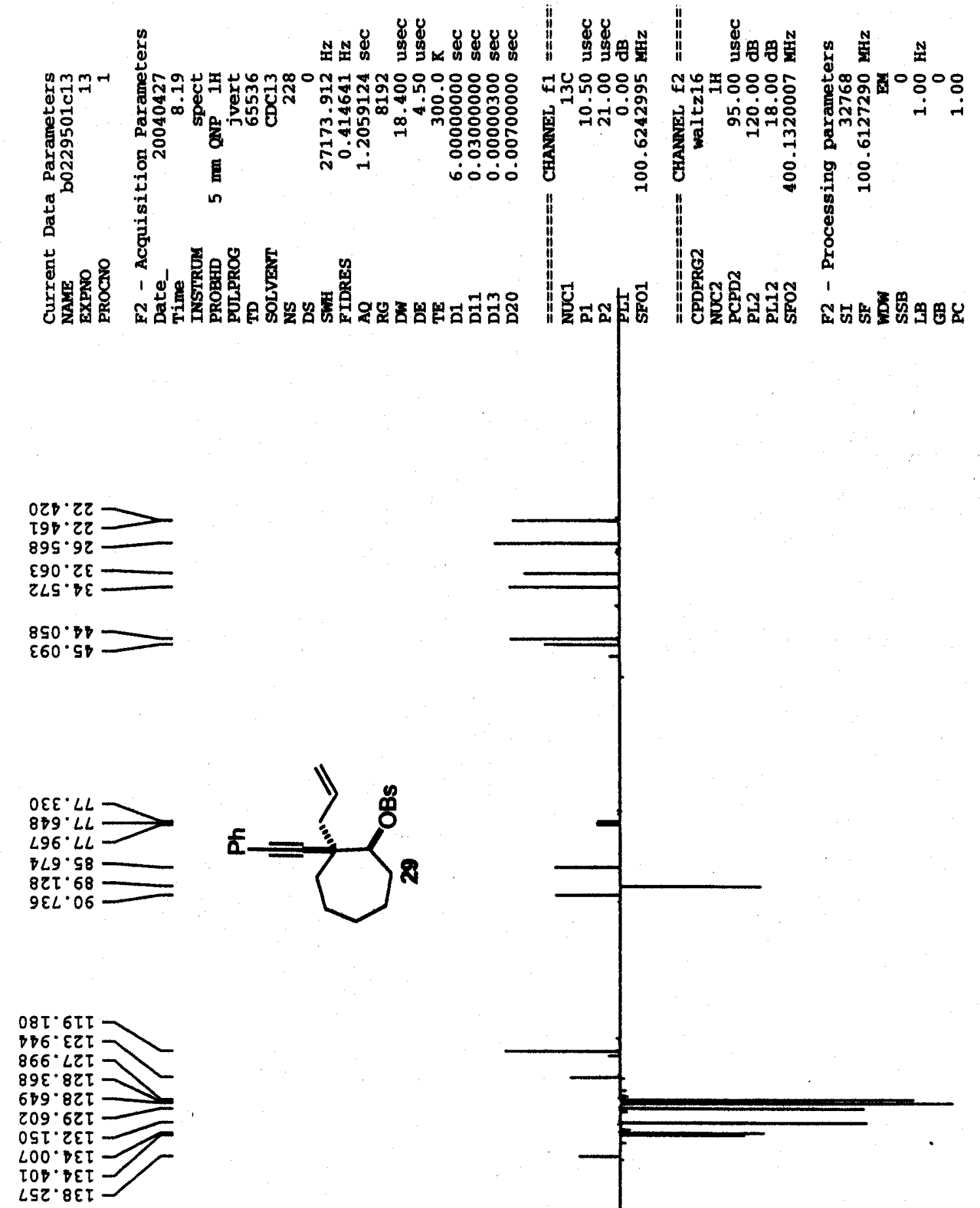

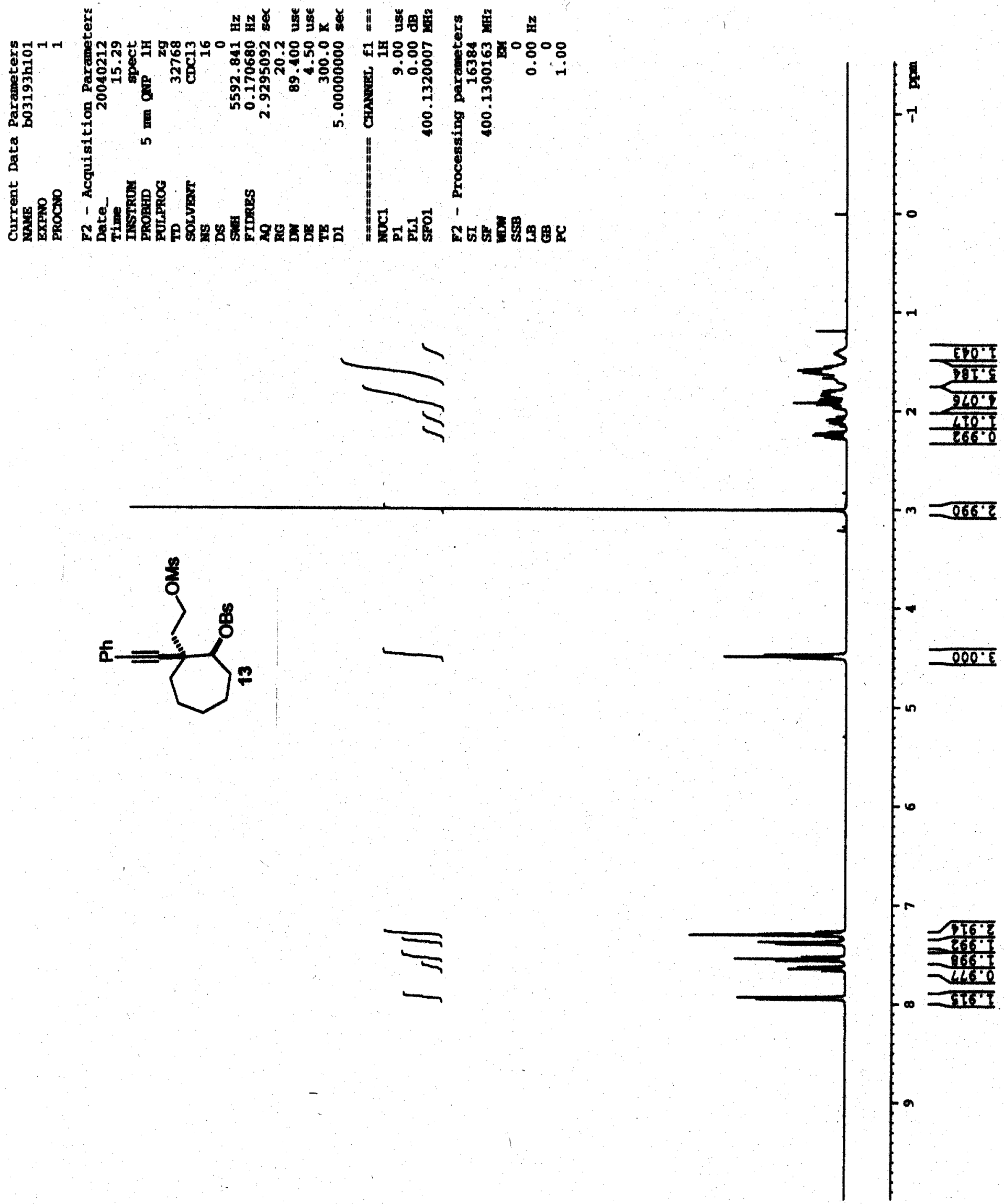

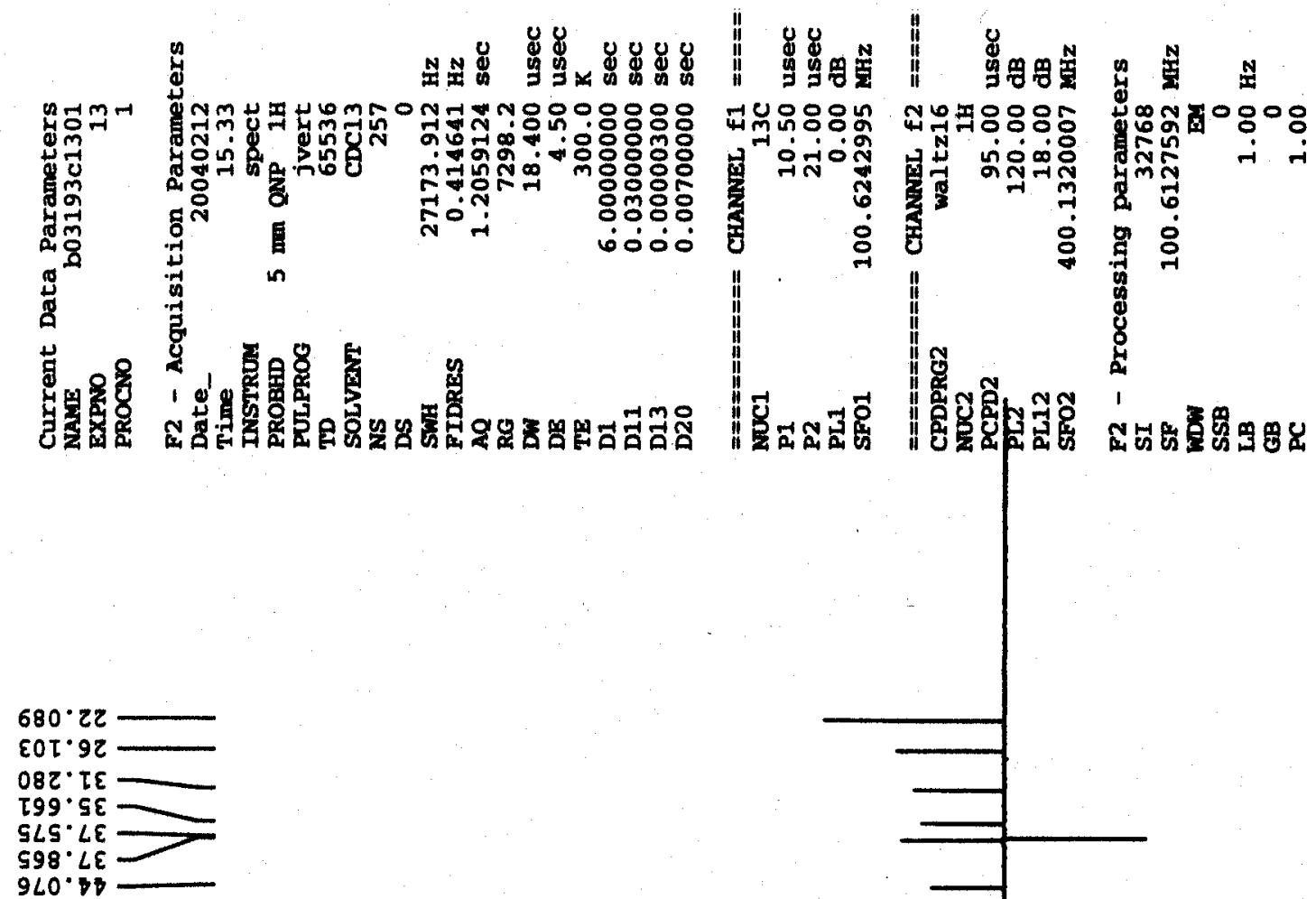

โ95" $\angle 9$

$9 \tau 6.9 L$

ZEZ'LL

$\angle 82 \cdot 98$

$\varepsilon 90 \cdot 88$

ILS. 88
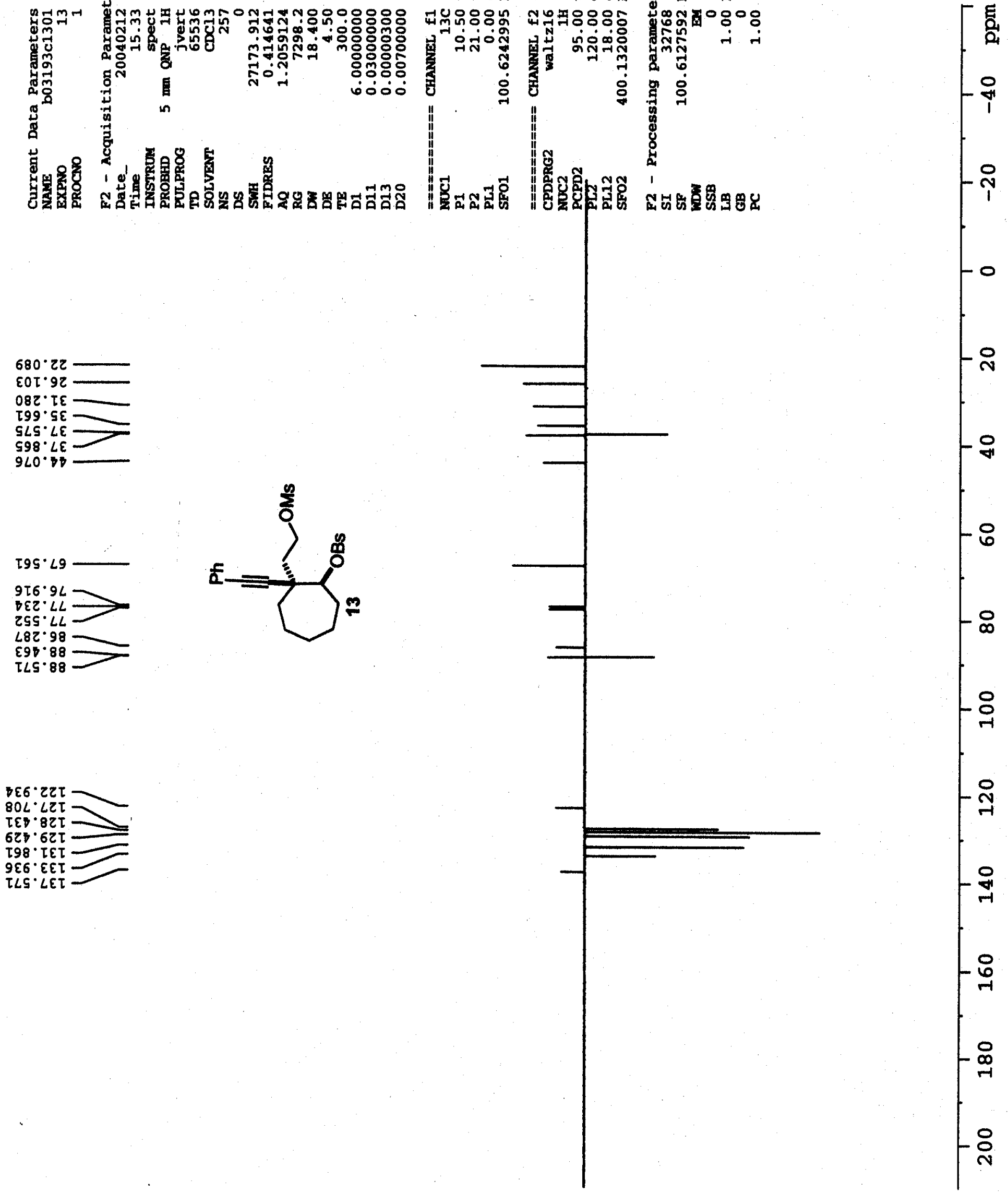

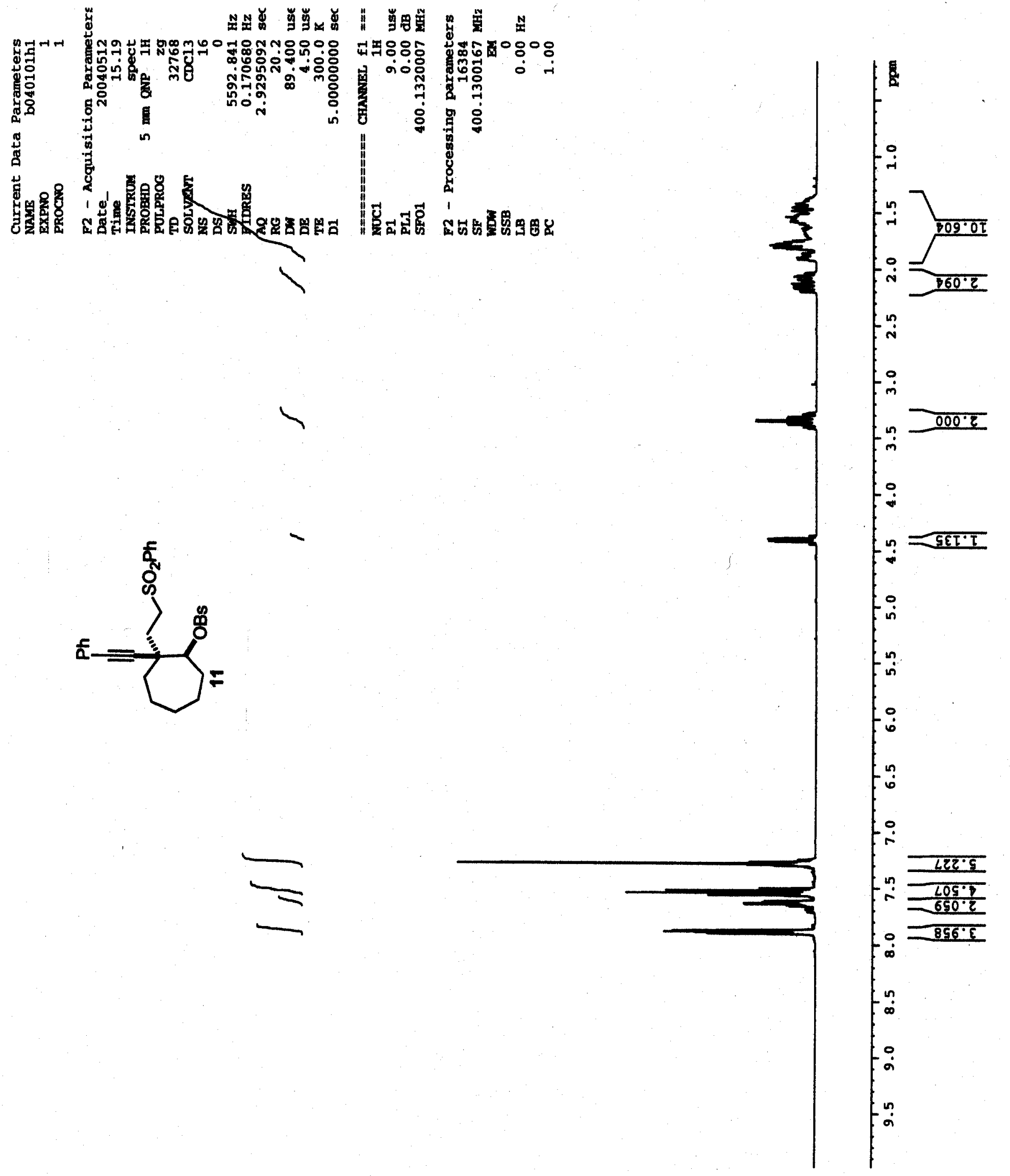

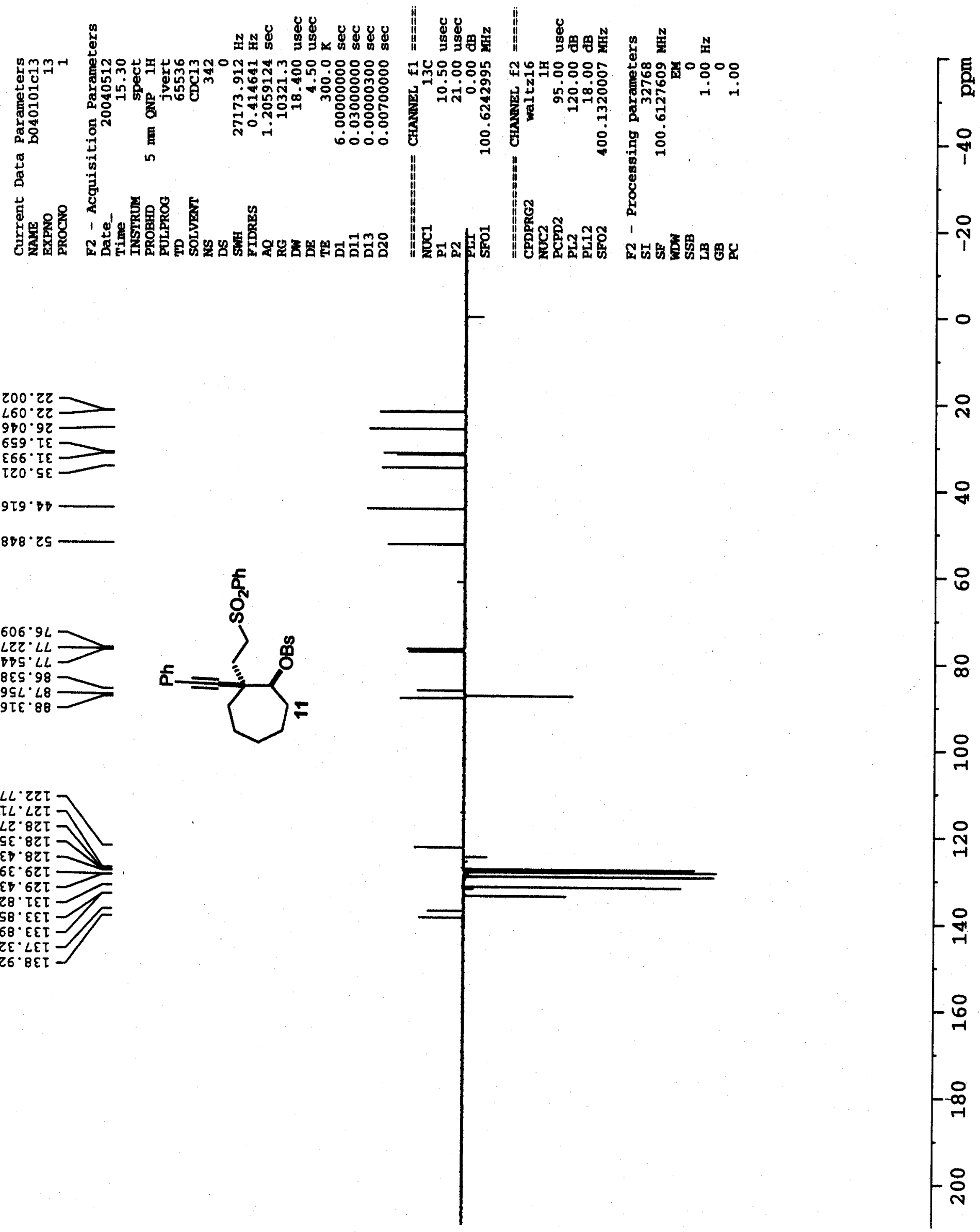

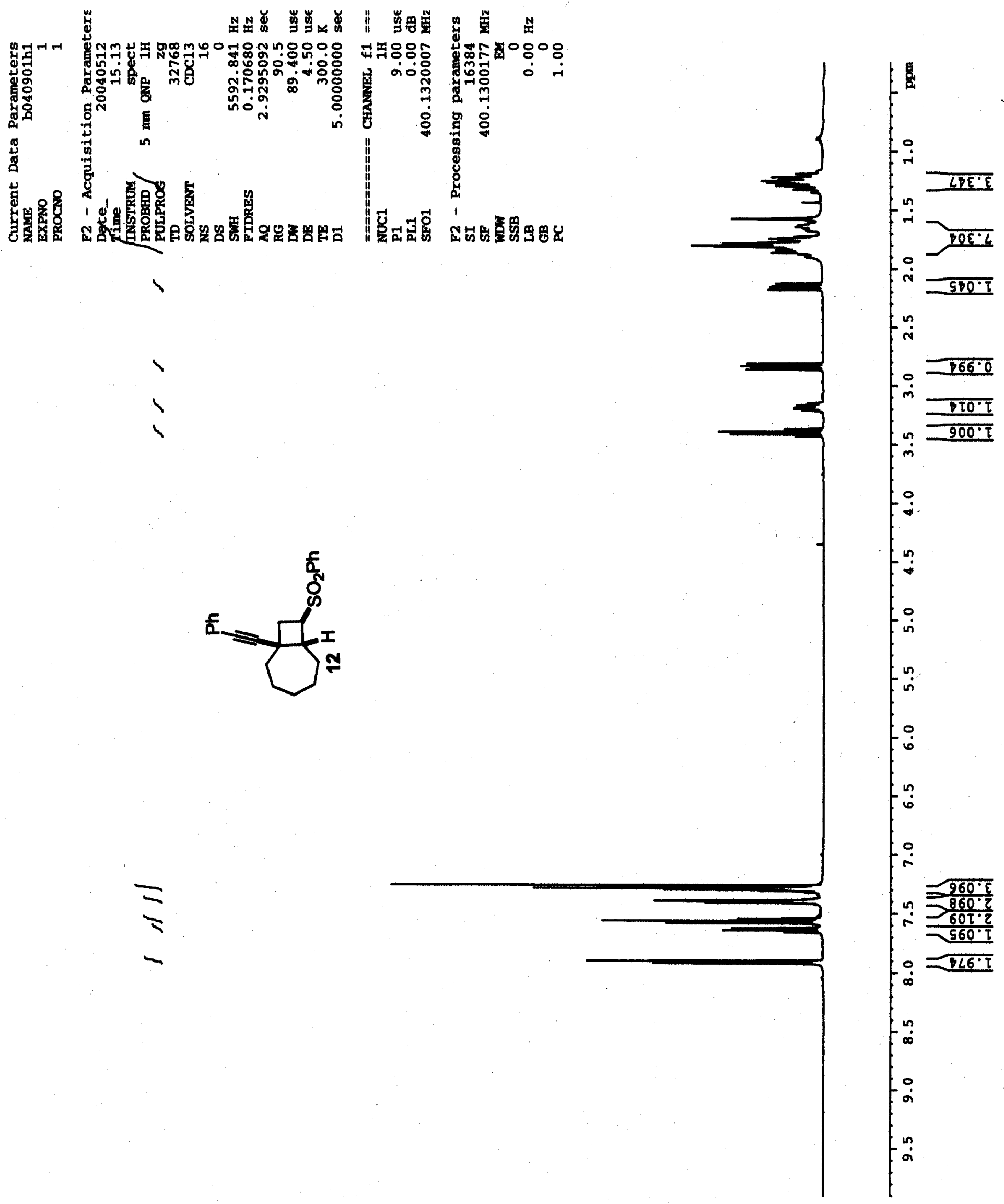

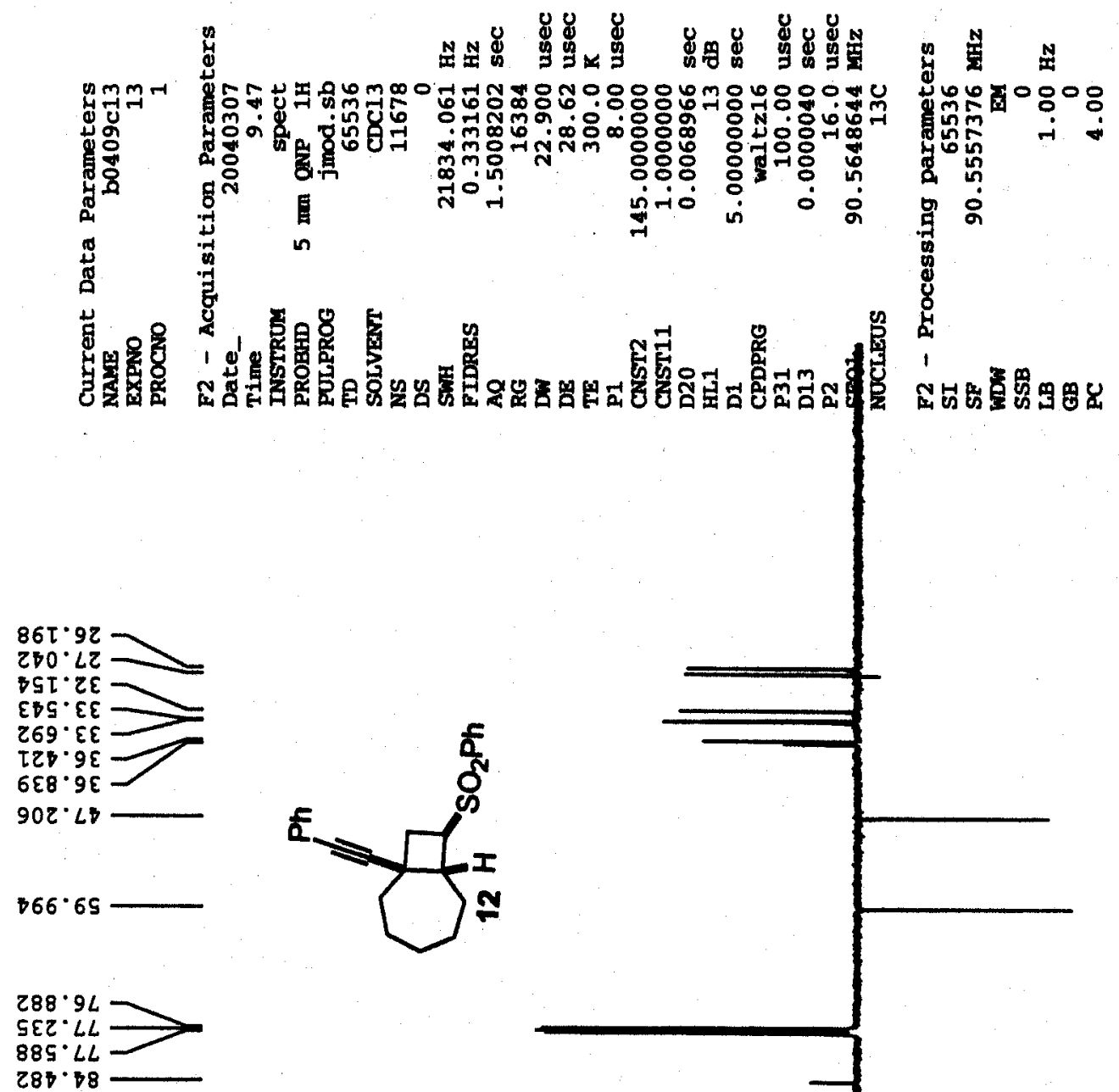

$0 \$ 56$
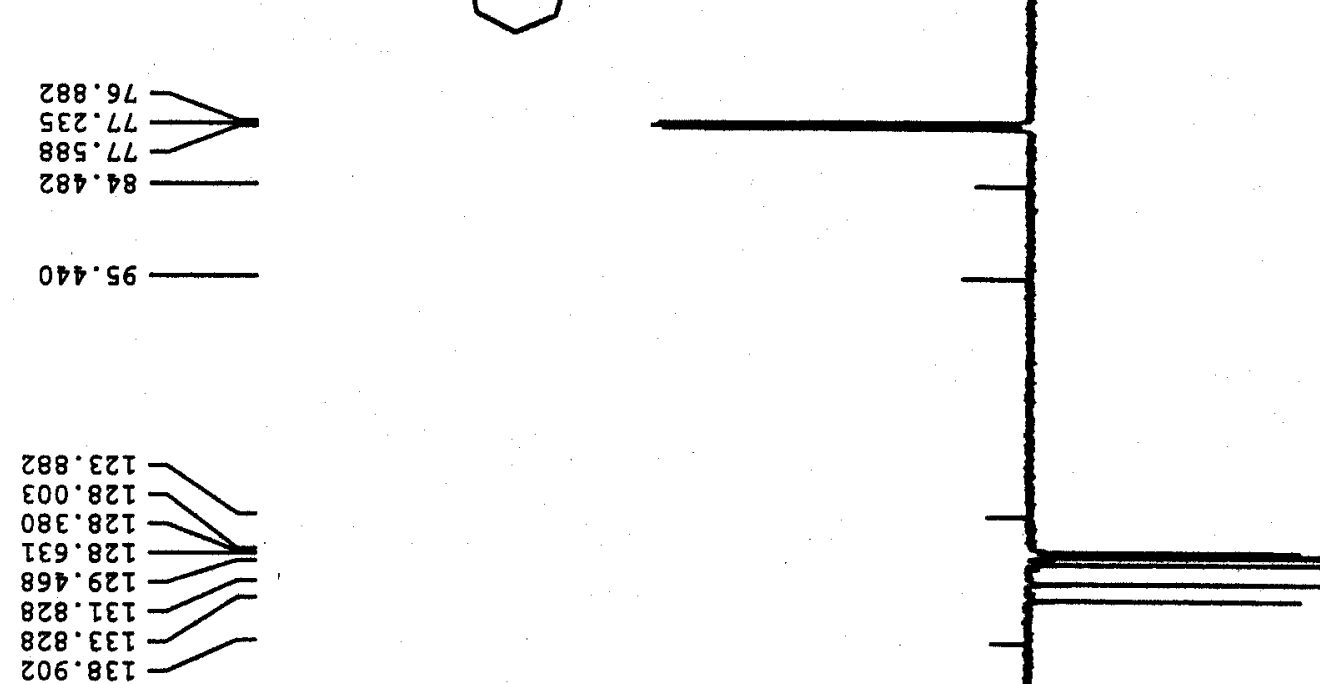

8

잉

욱

국

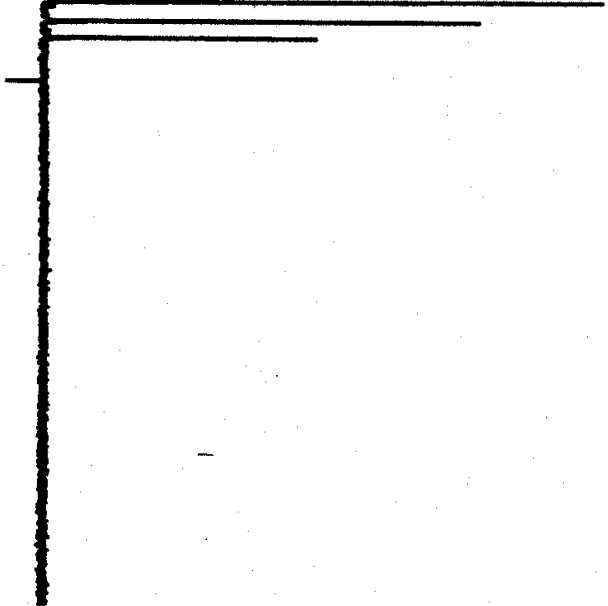



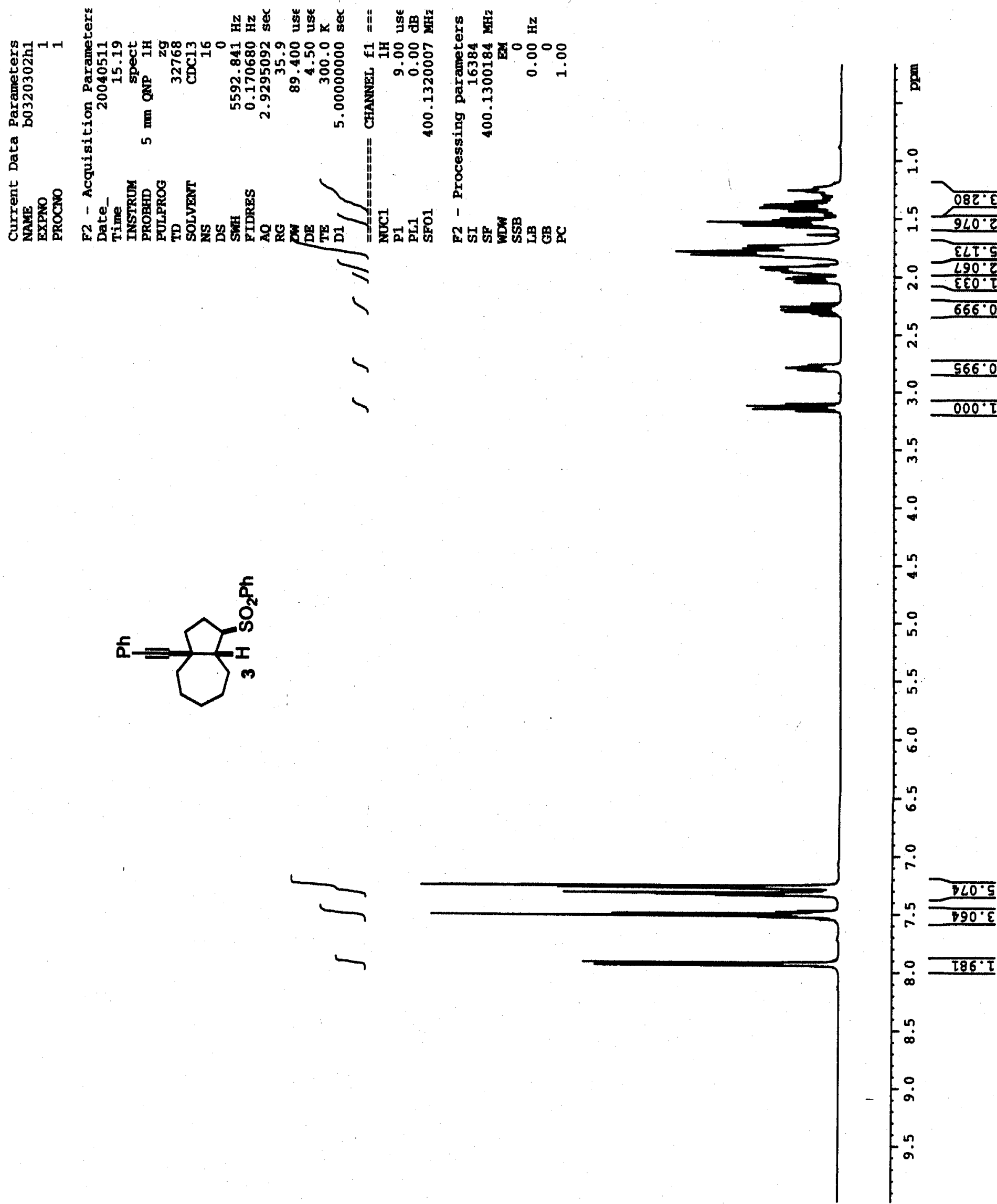

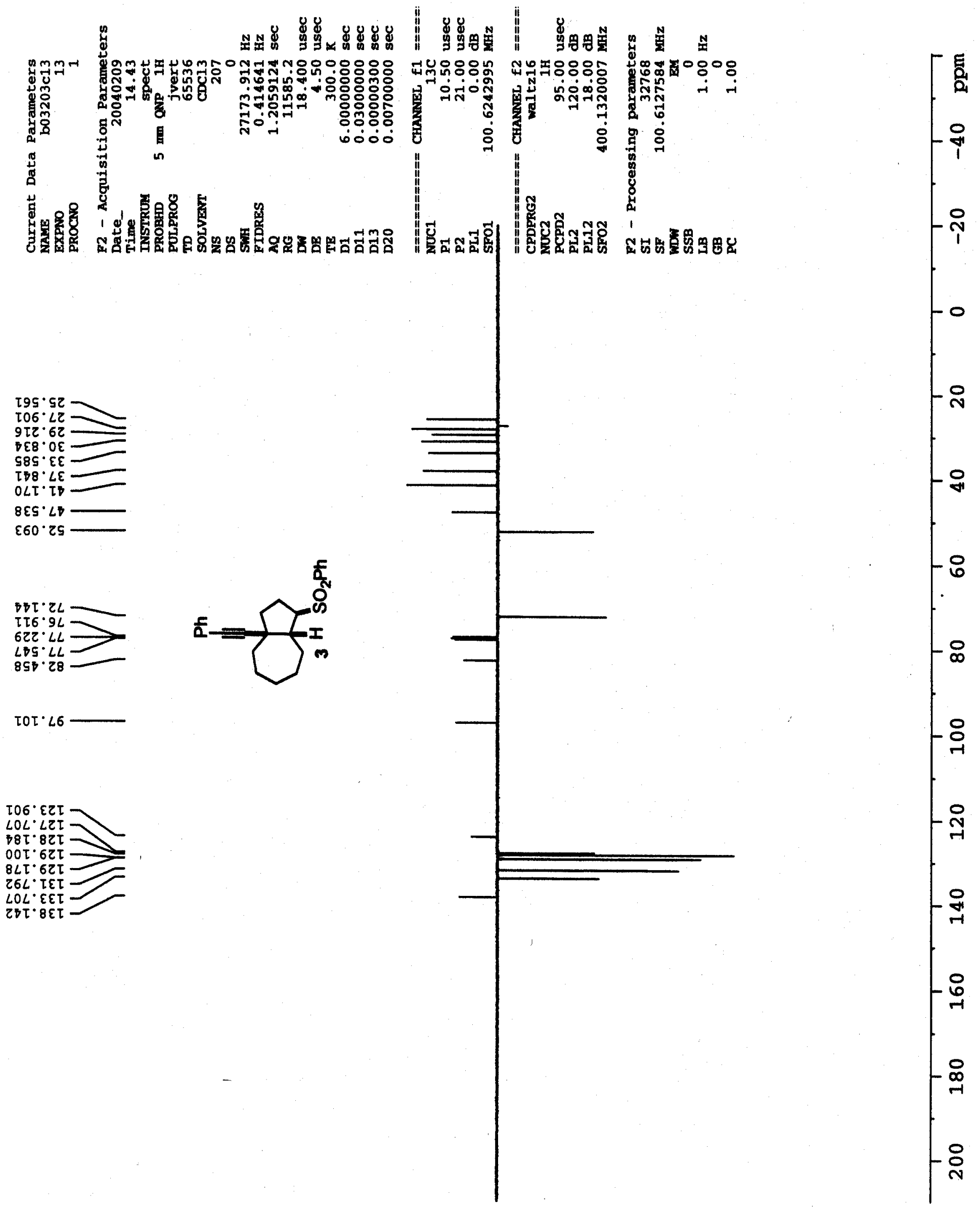

TOT:L6
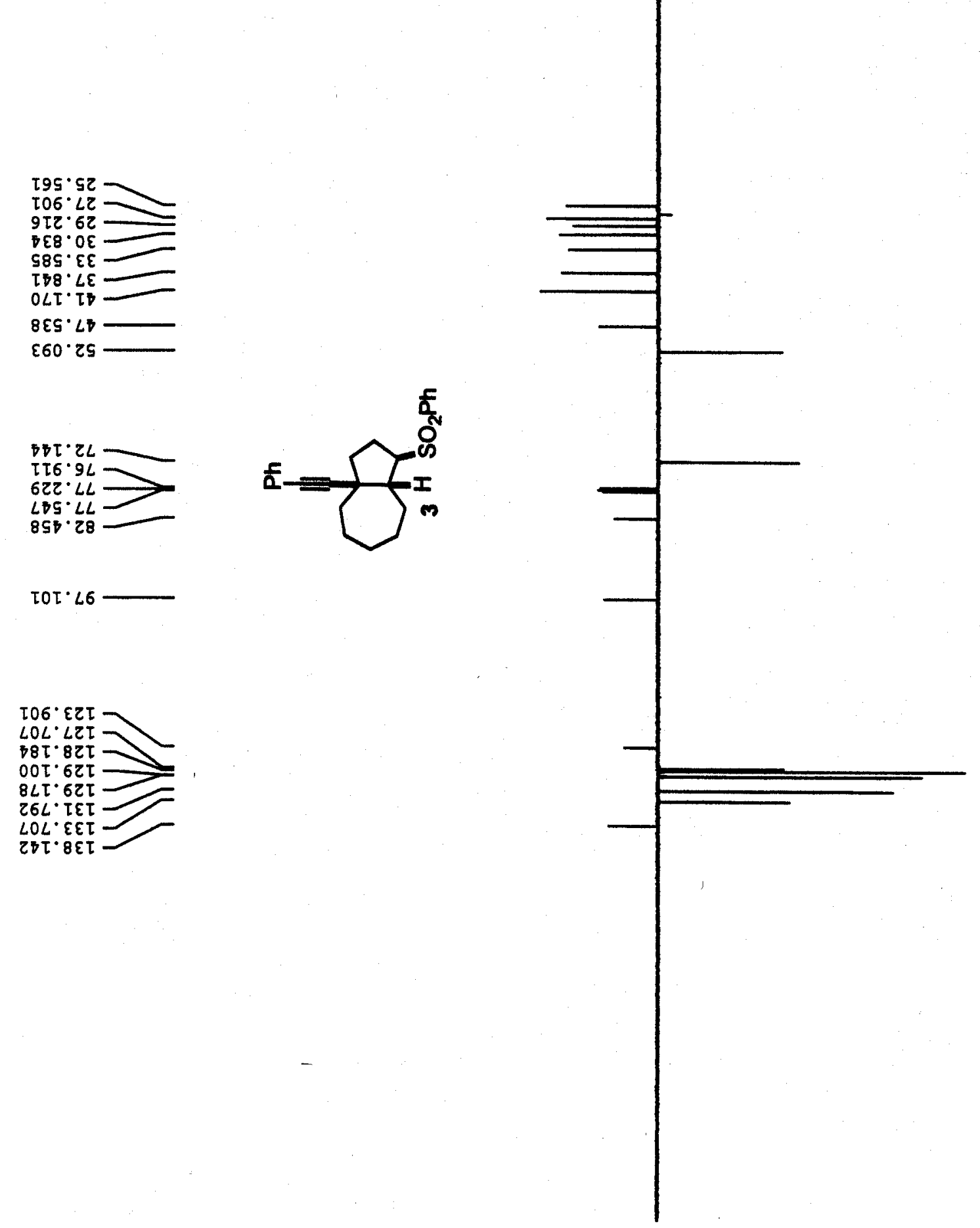


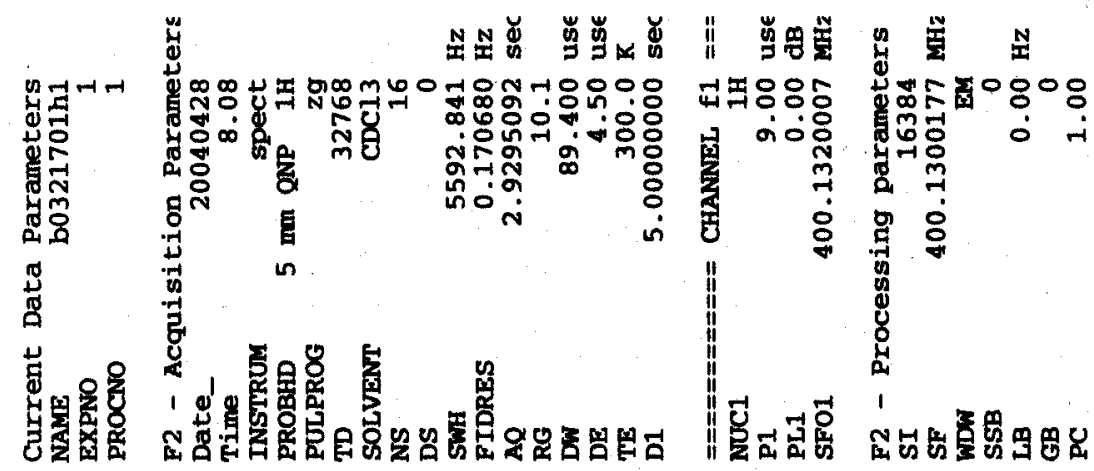

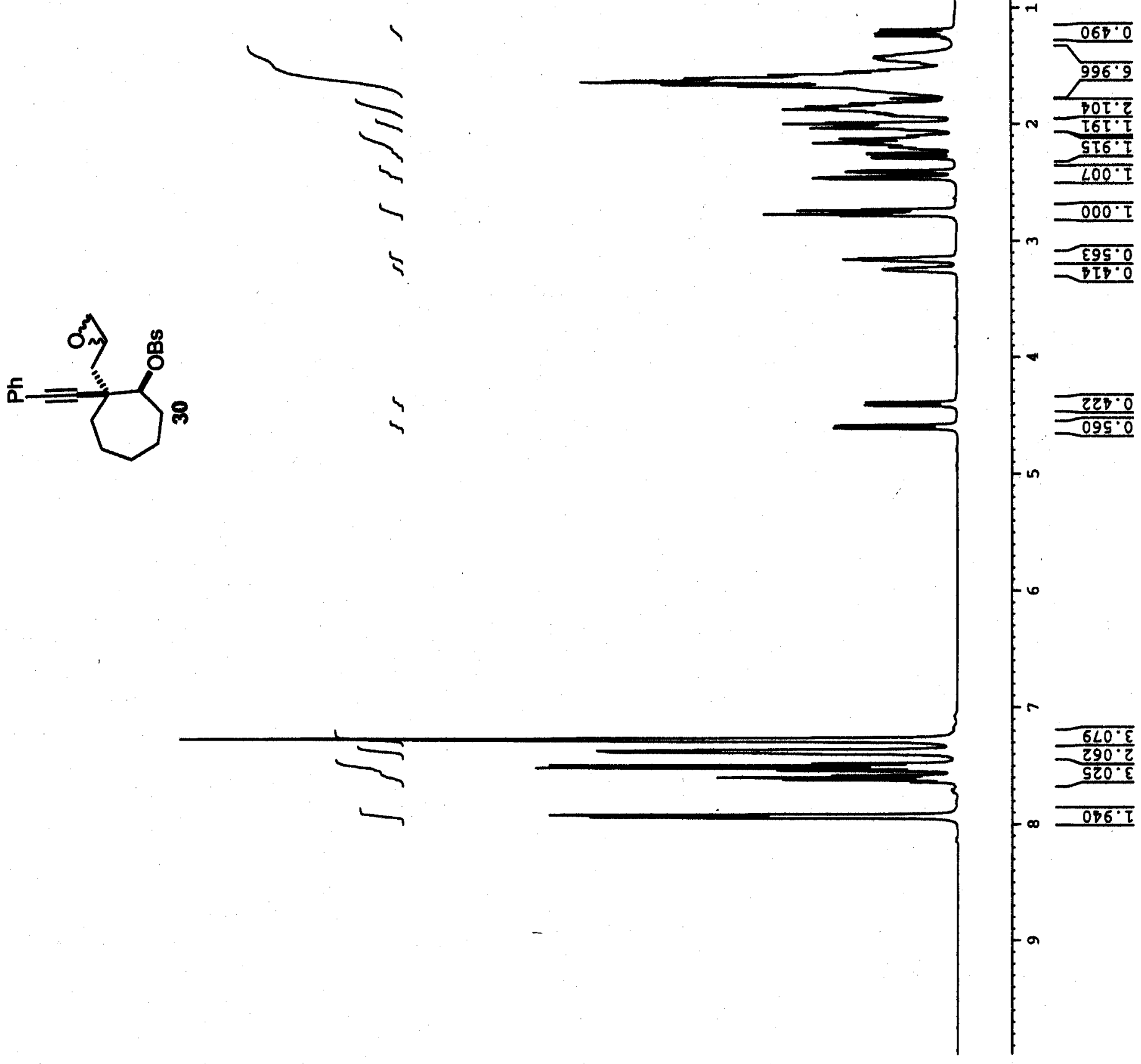



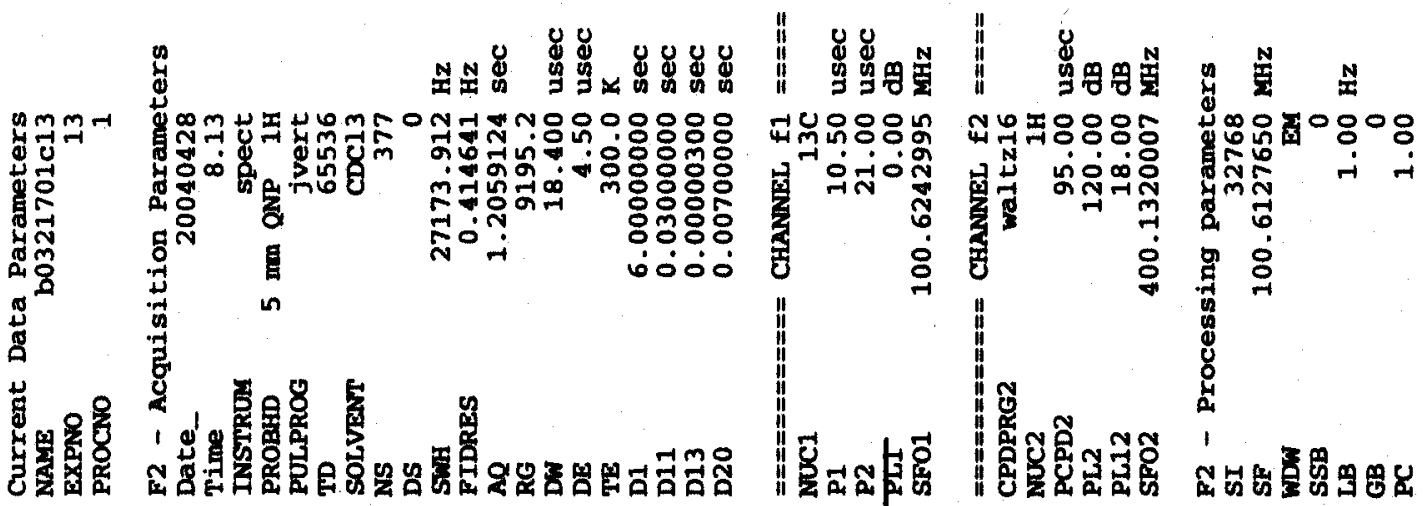

$\left[\begin{array}{l}0 \\ 0 \\ 0 \\ 0 \\ 1 \\ 0 \\ 0 \\ 1\end{array}\right.$
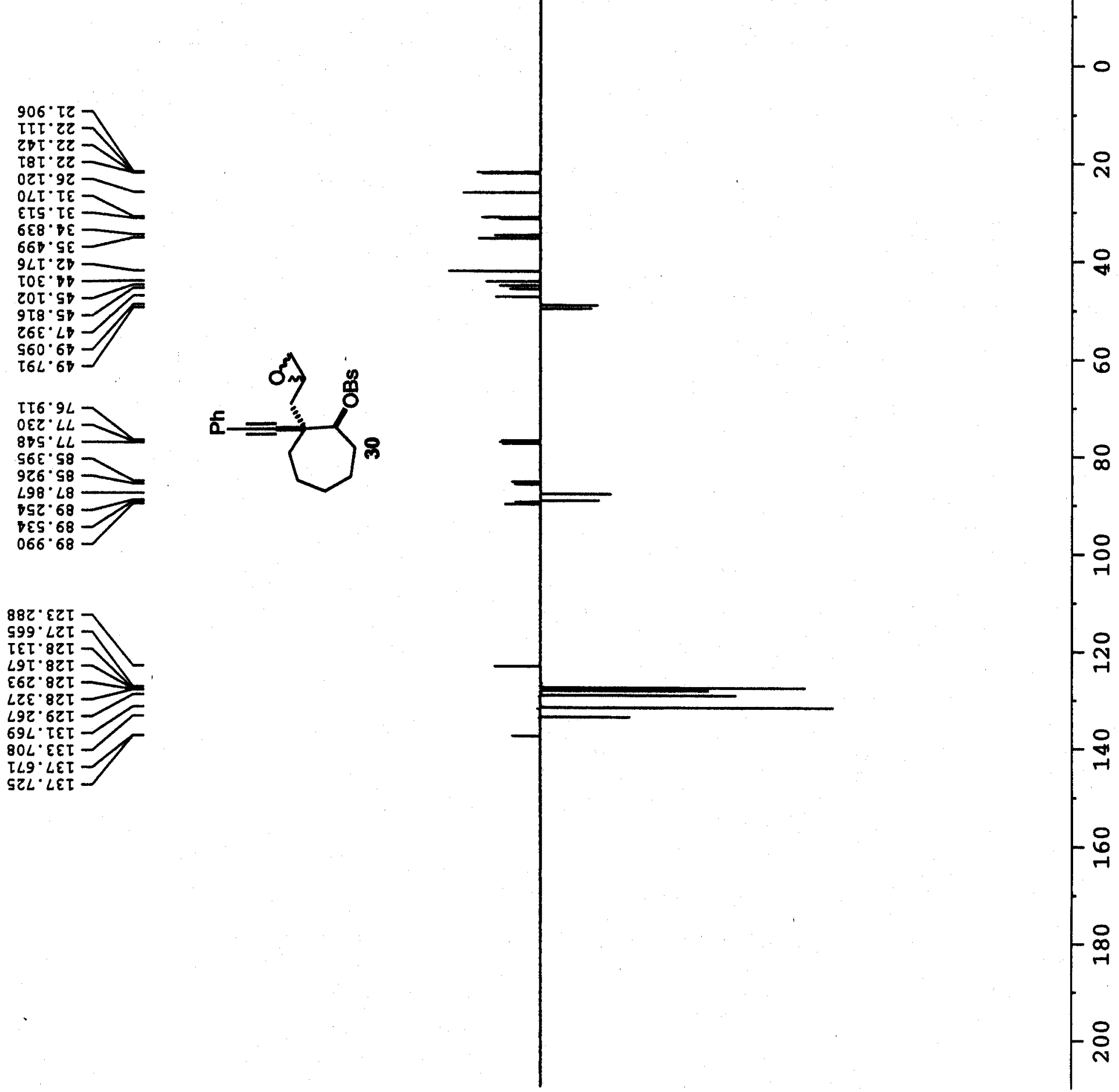

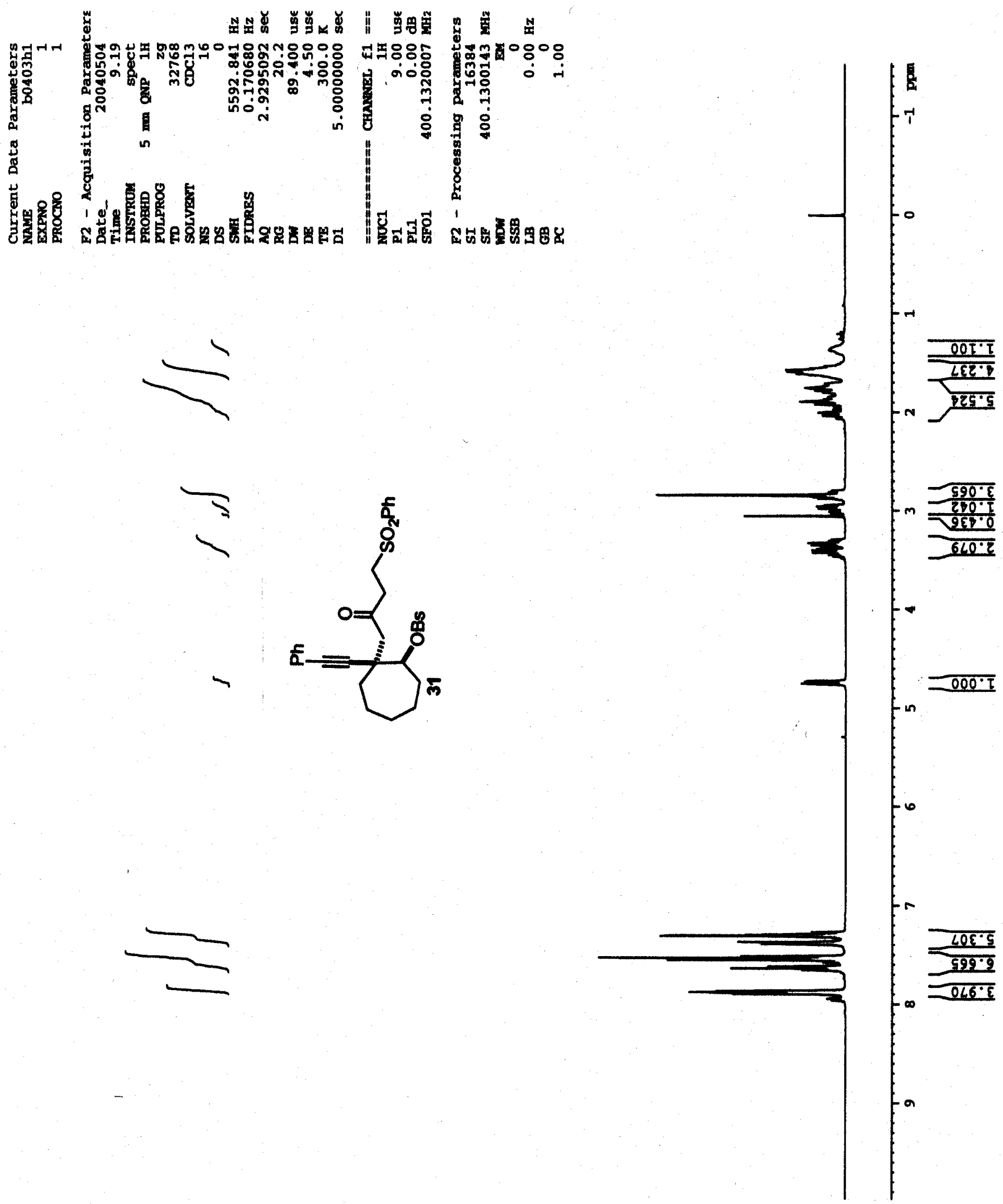

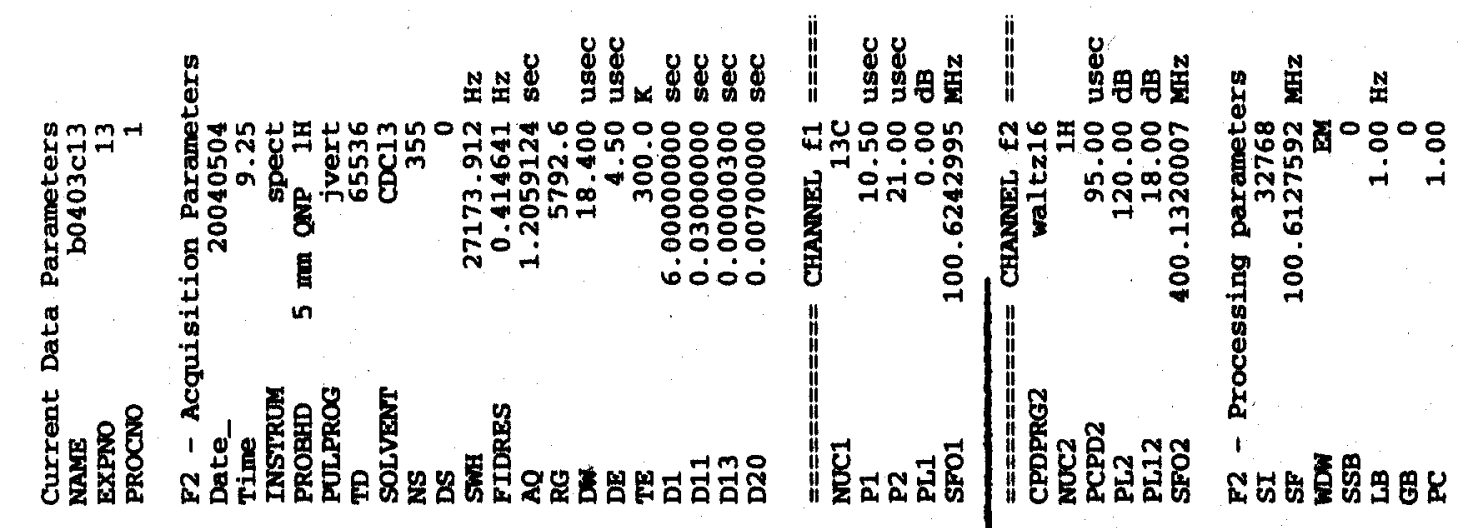

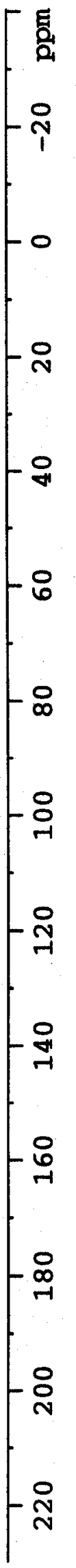
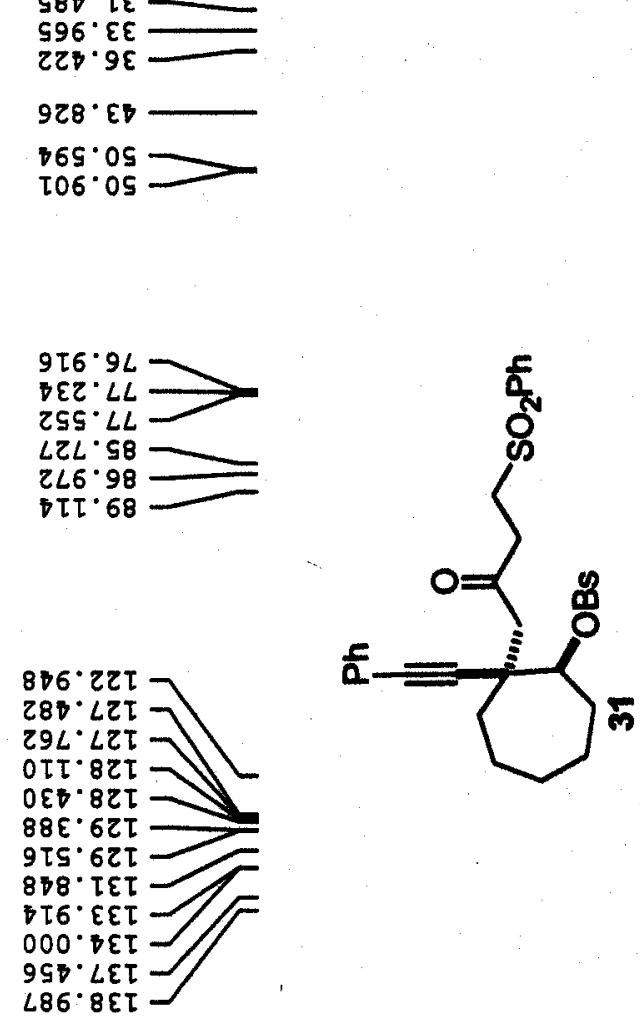

$O B L \cdot \varepsilon O Z$

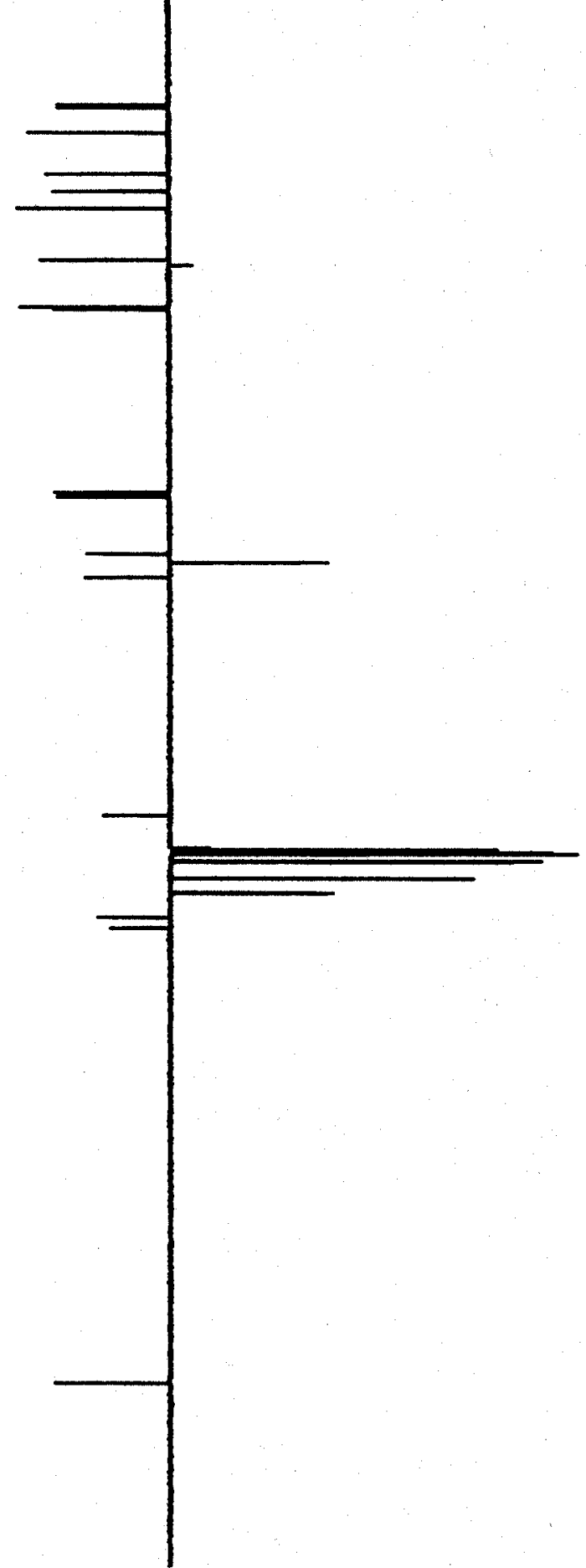

우

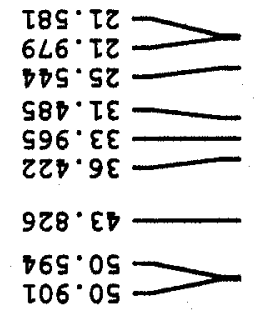



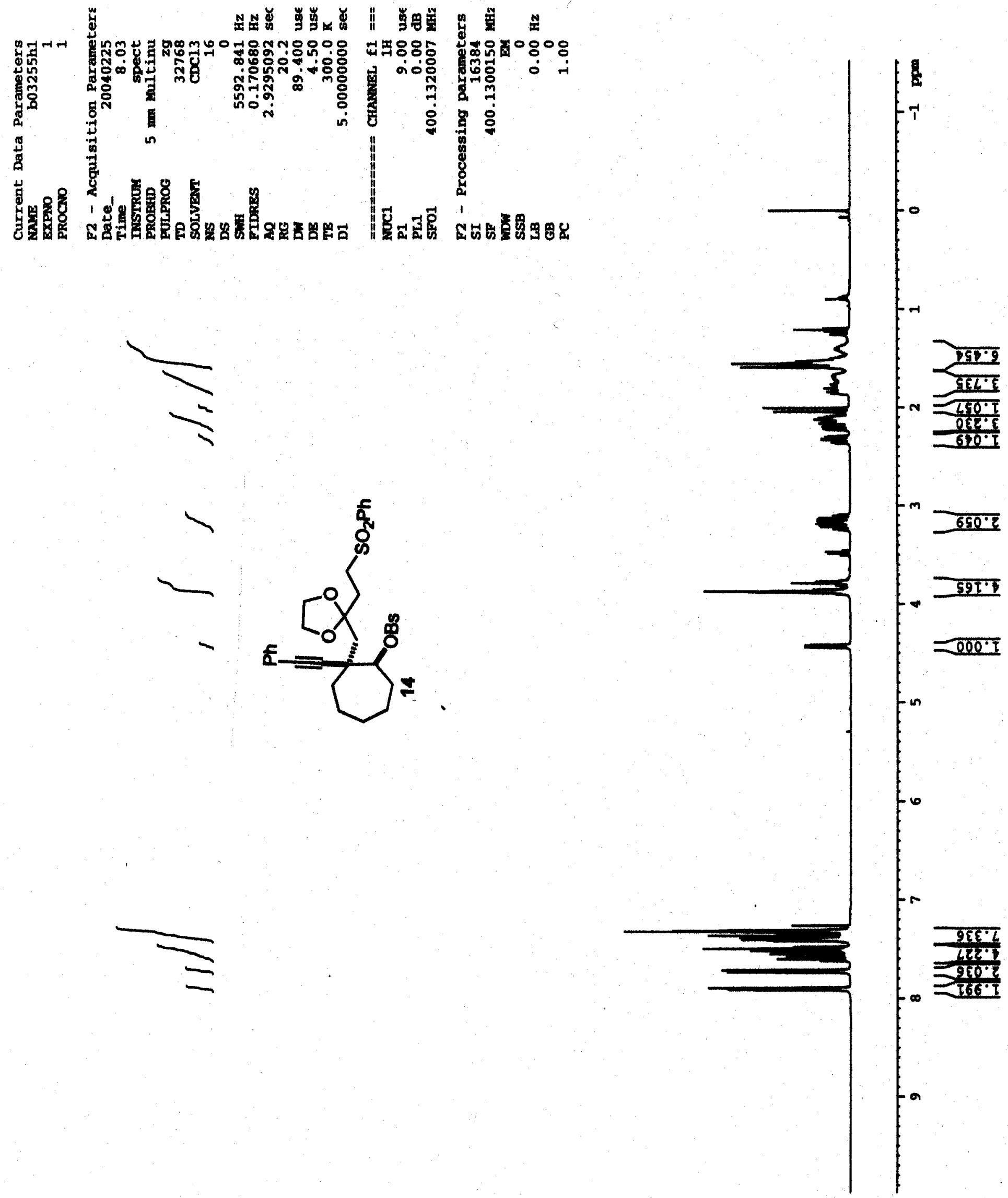

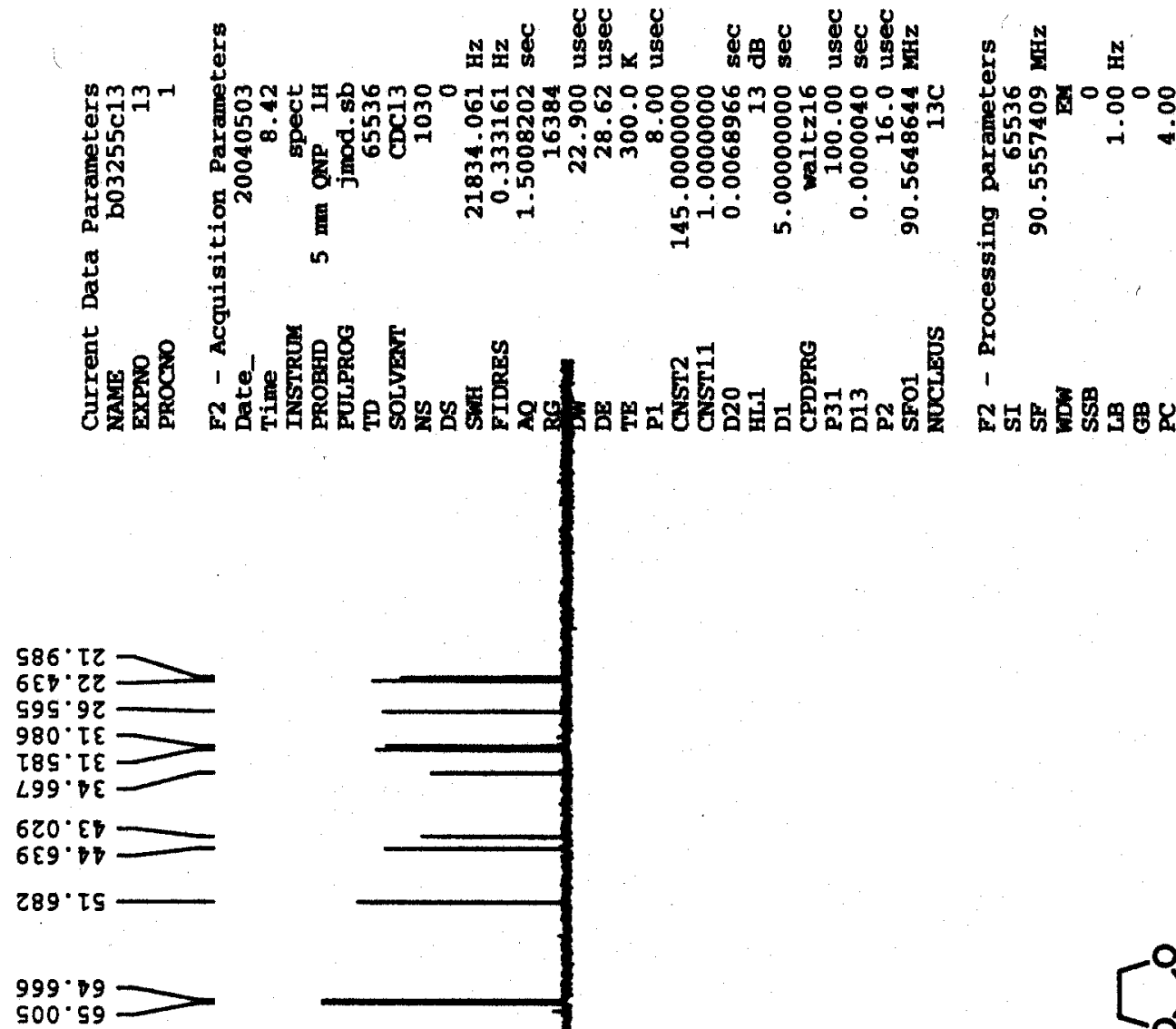

$9 L 8^{\circ} 9 L$
$622 \cdot L L$ Z85' $L L$

$599^{\circ} 58$

$298^{\circ} 68$

90L:06
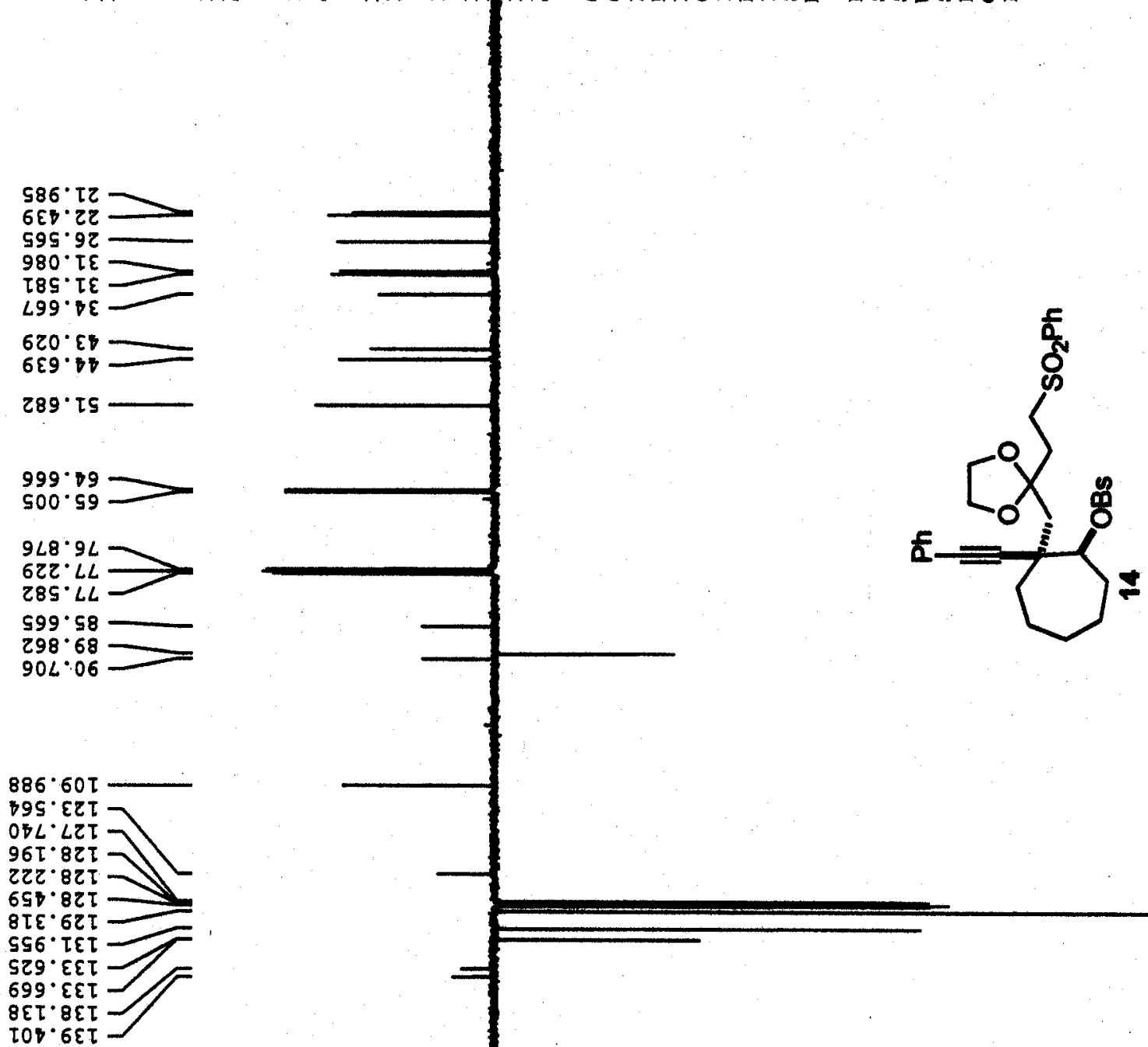

i

운

8

$-\infty$

$\underset{-1}{0}$

욱

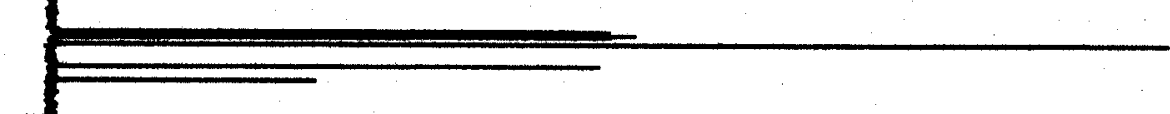

욤

$\underset{-1}{-1}$

옴 

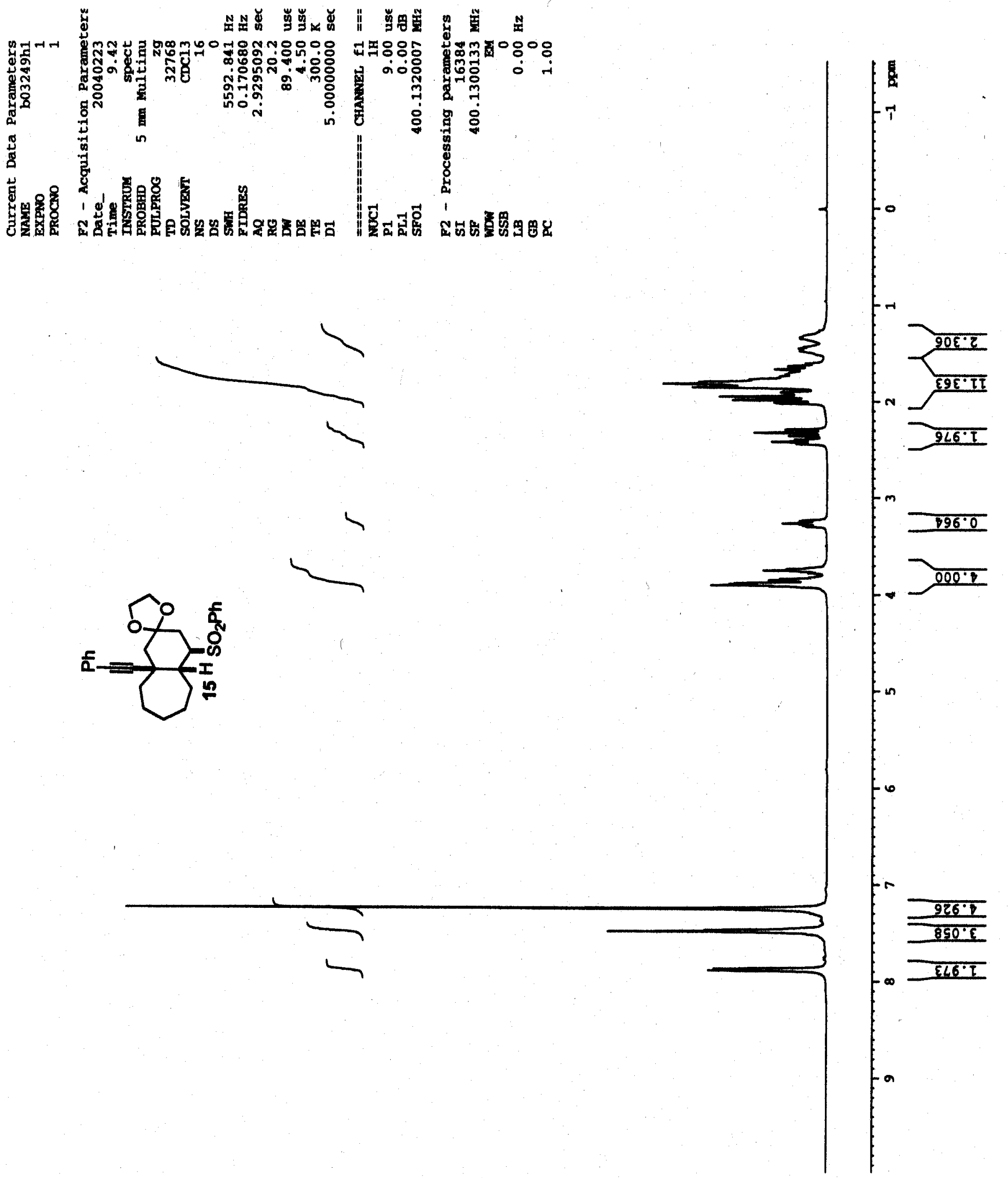

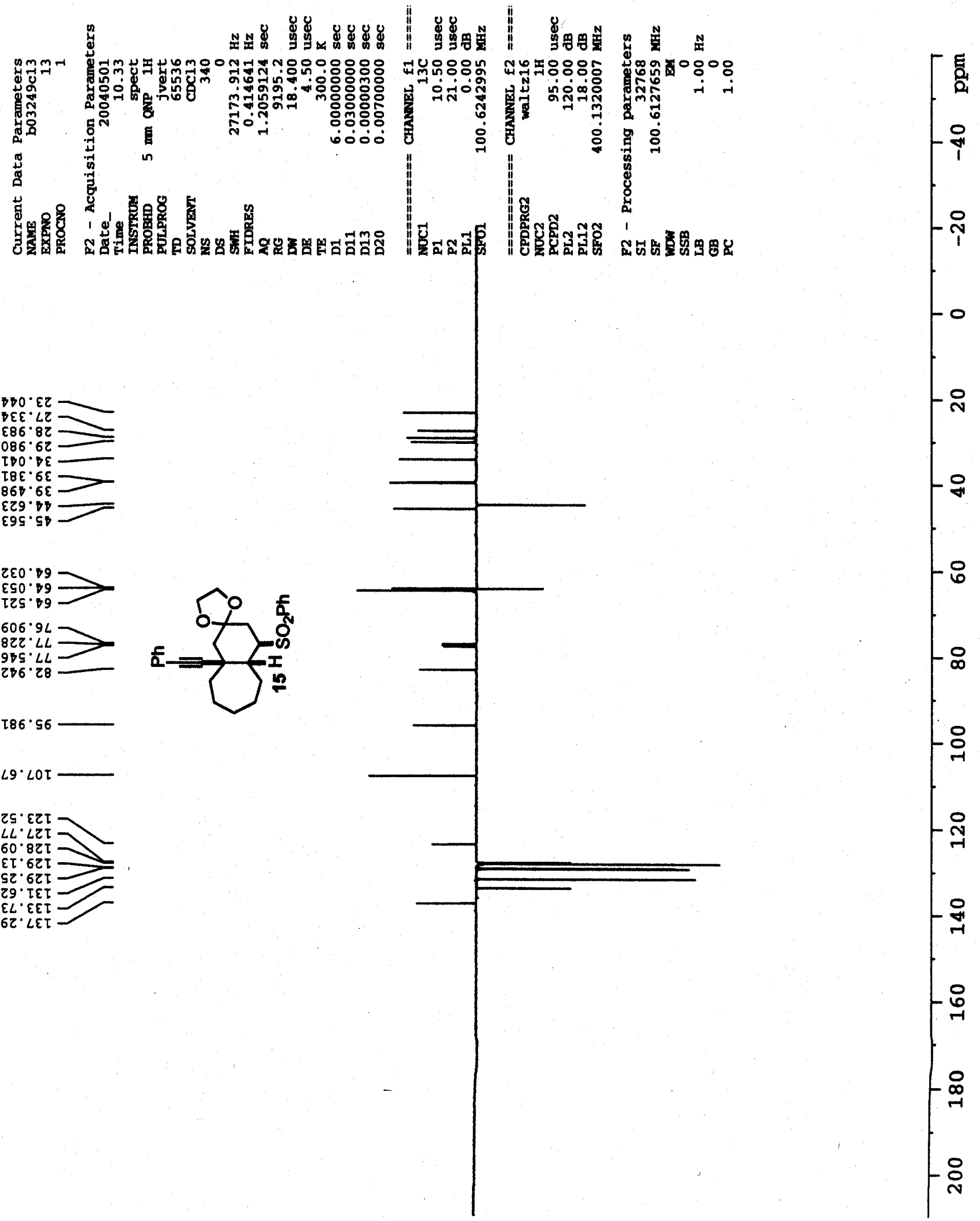

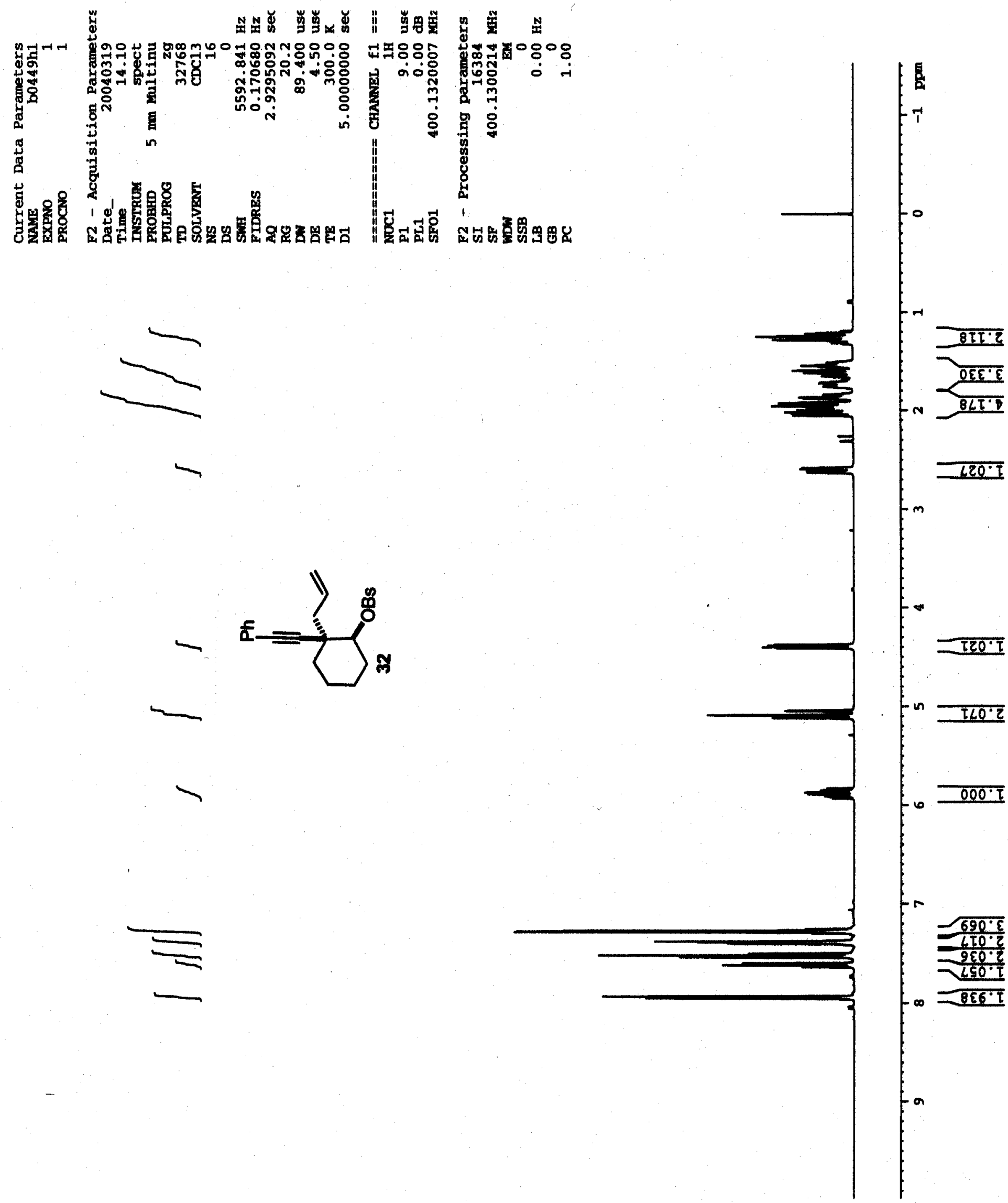

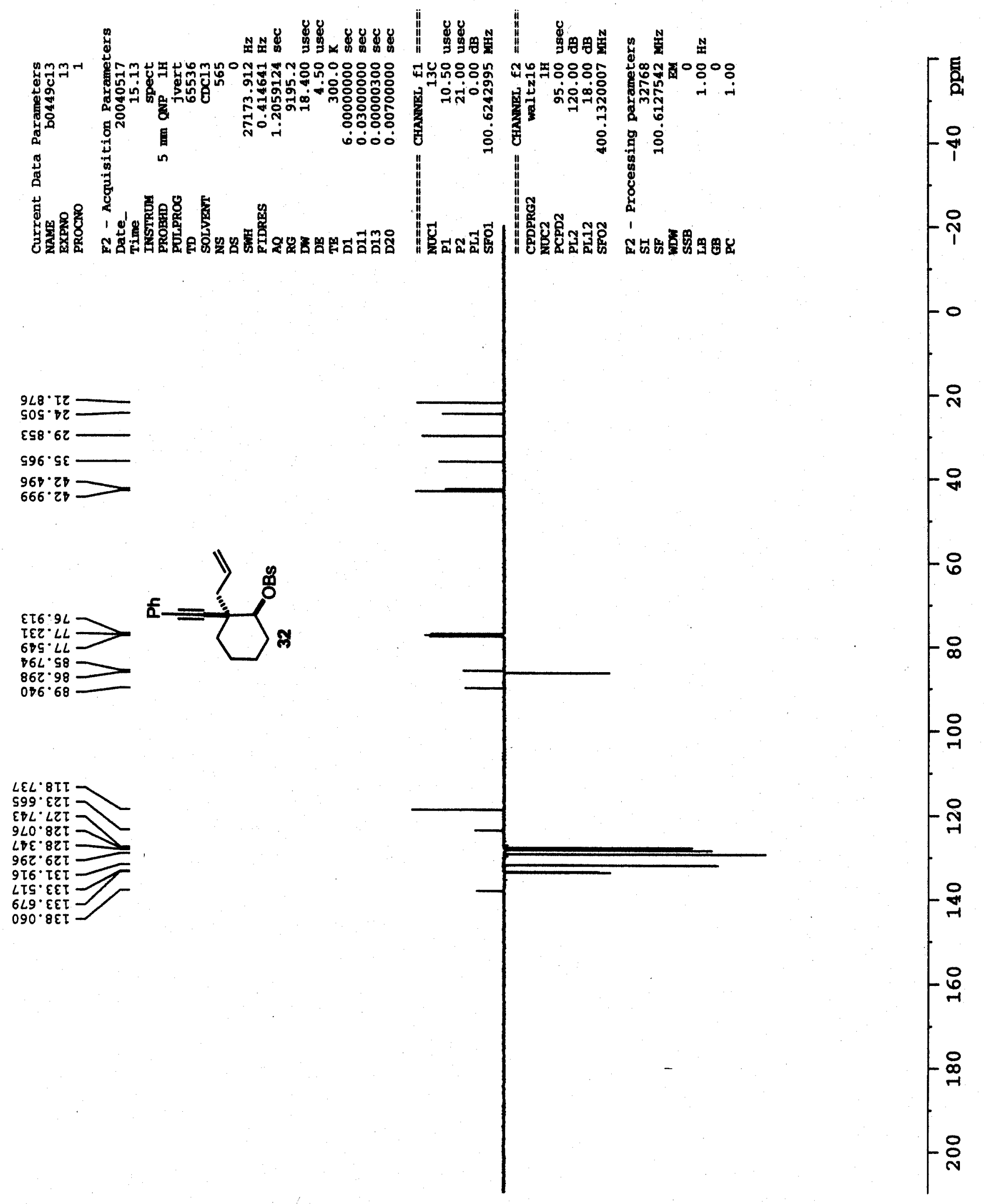

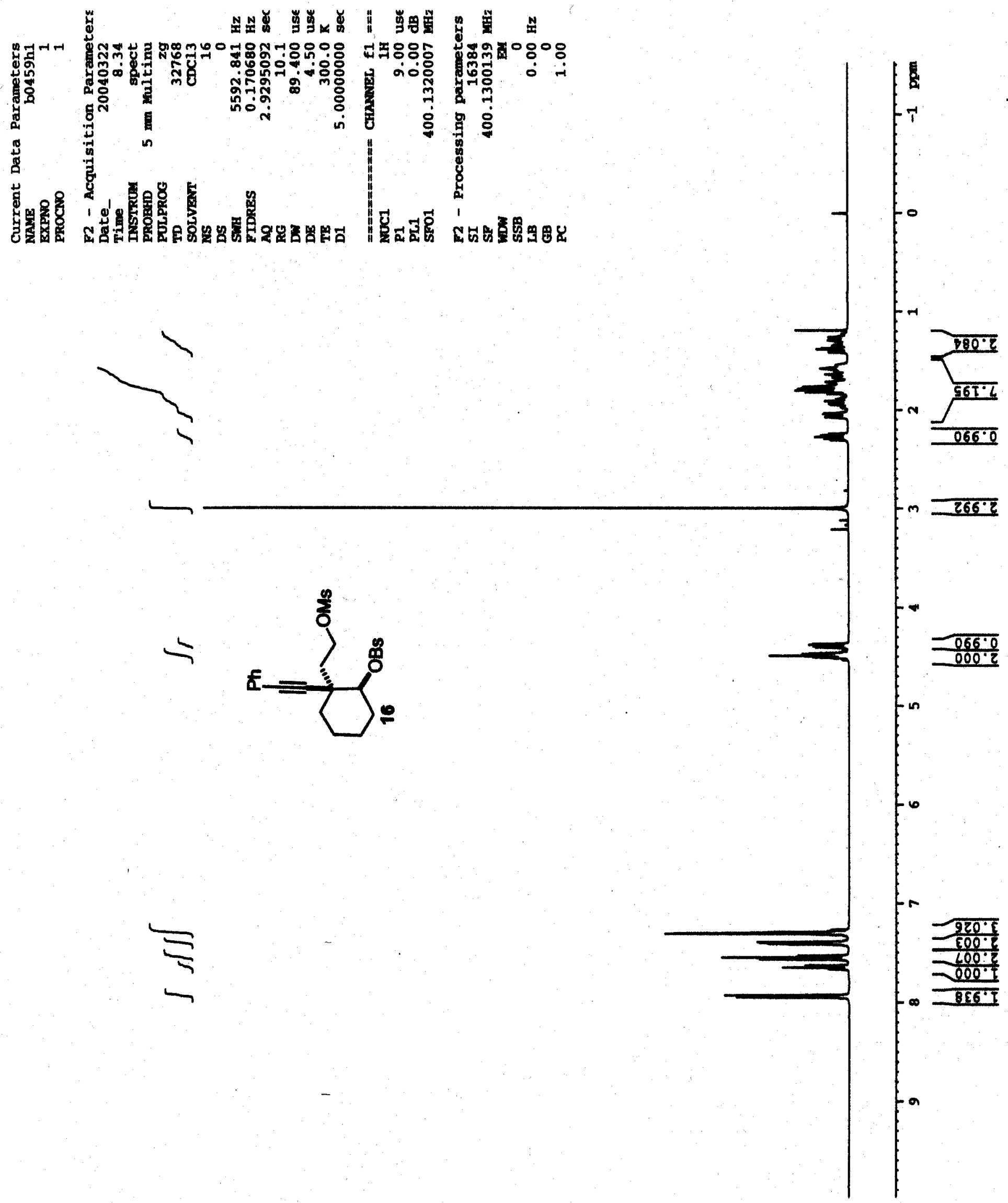

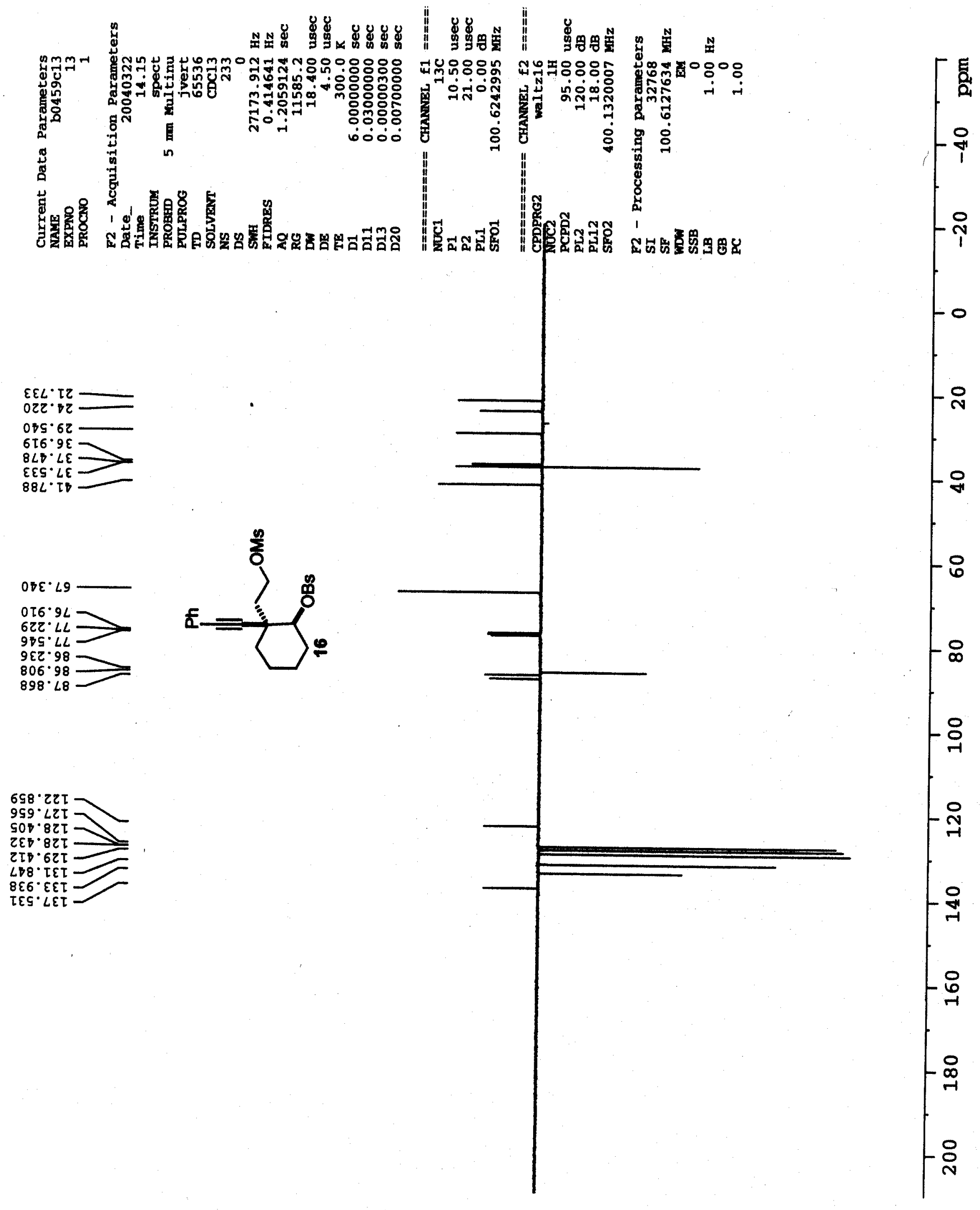

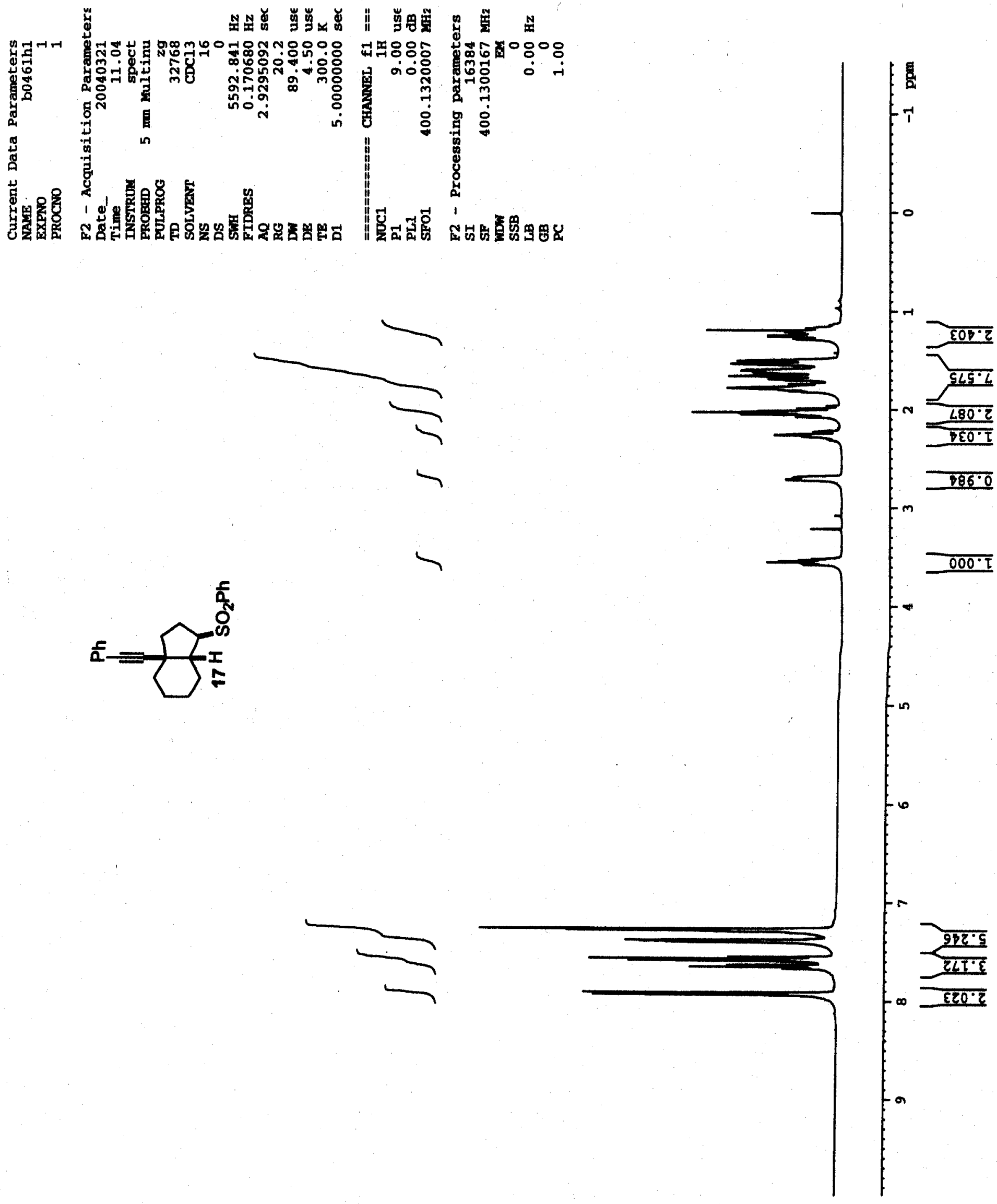

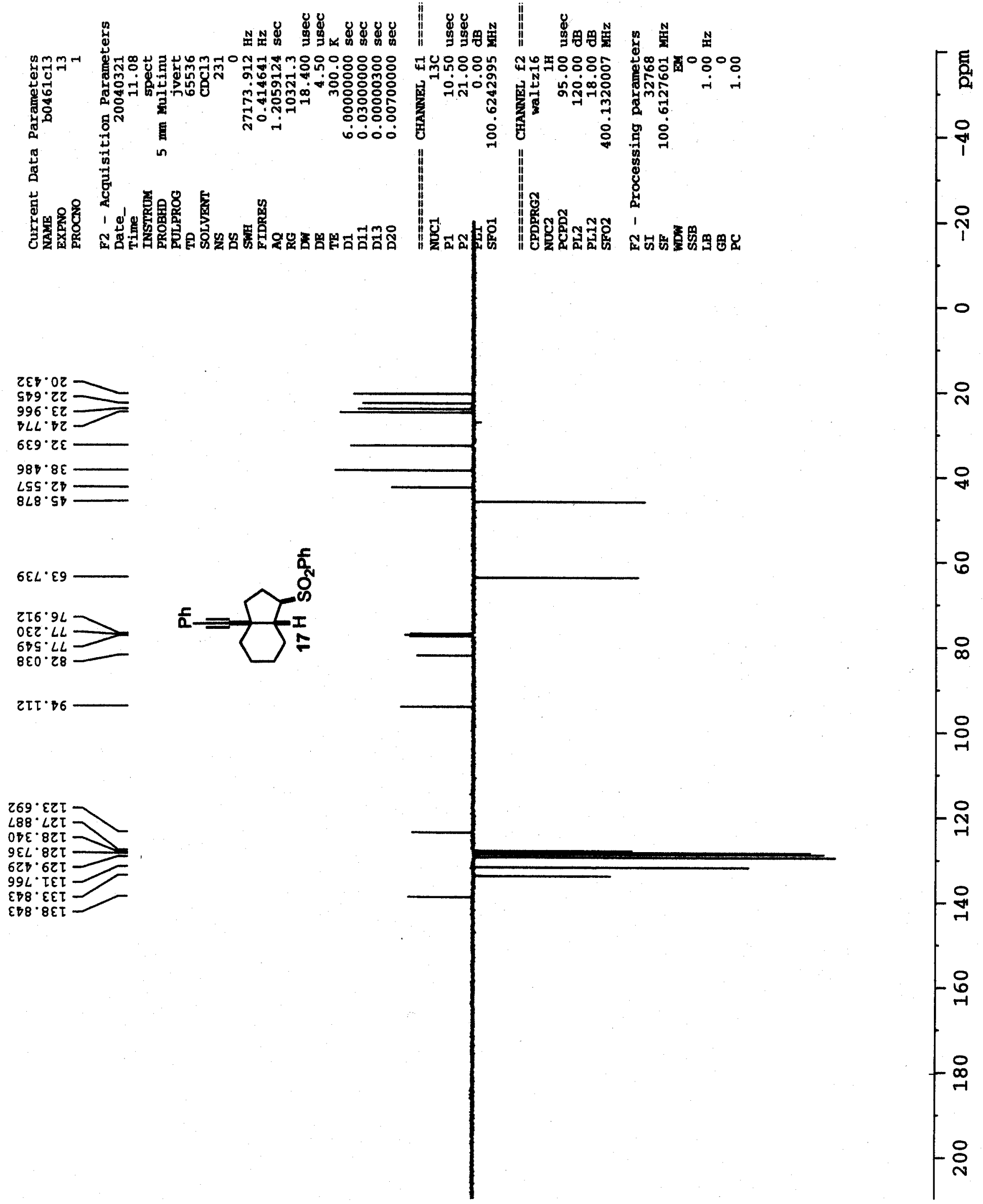

$2 \tau \tau \cdot \nabla 6$
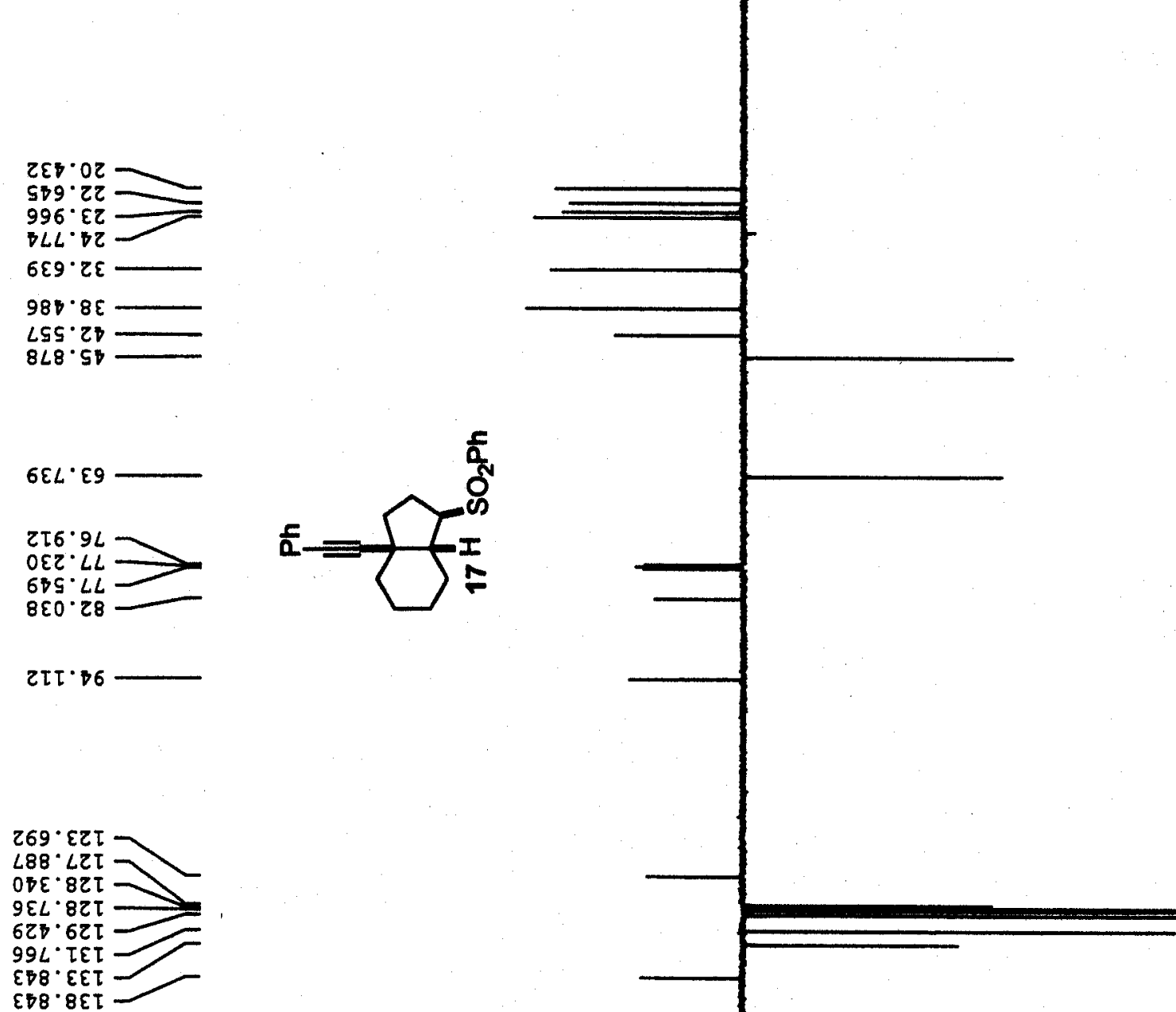

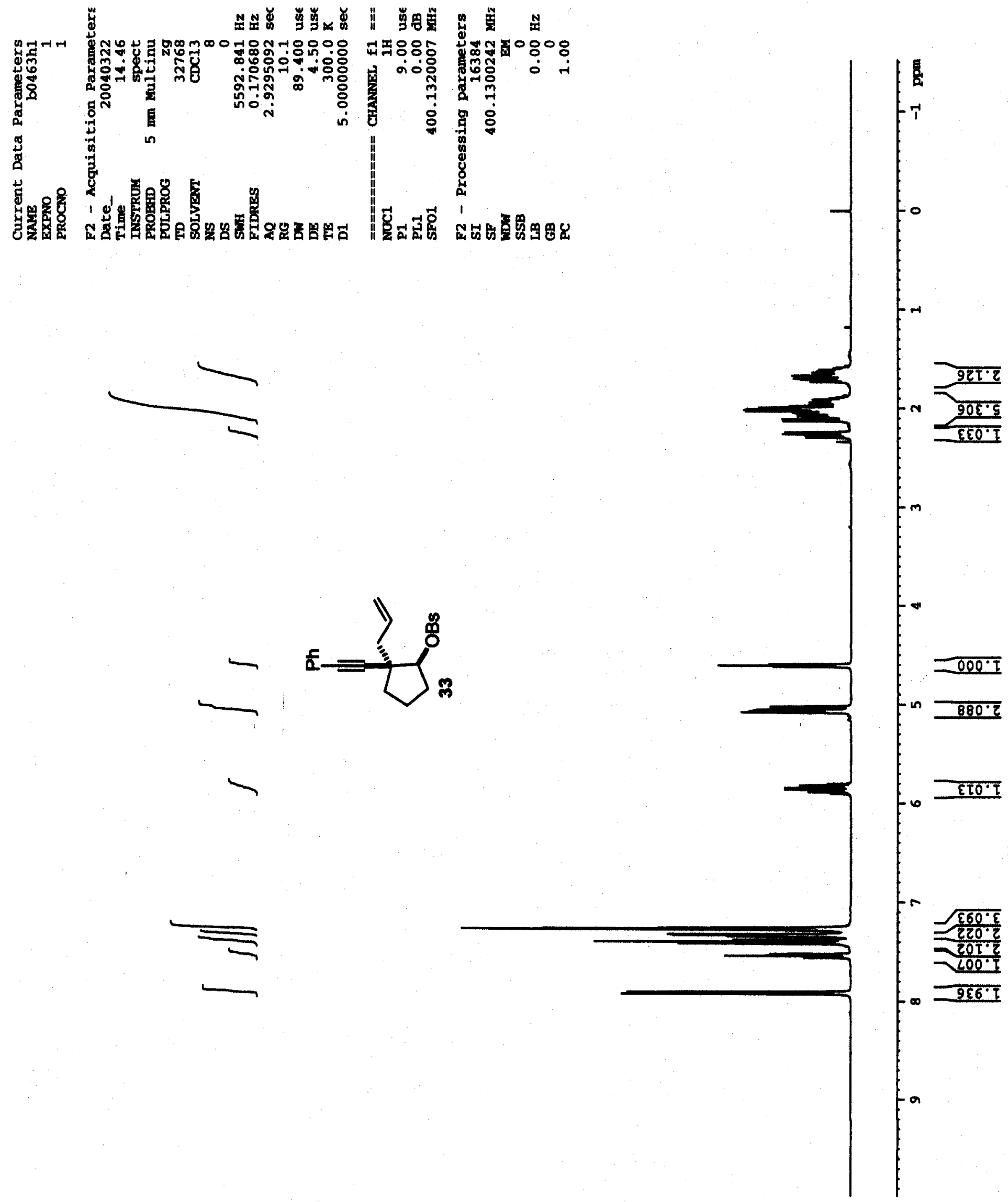

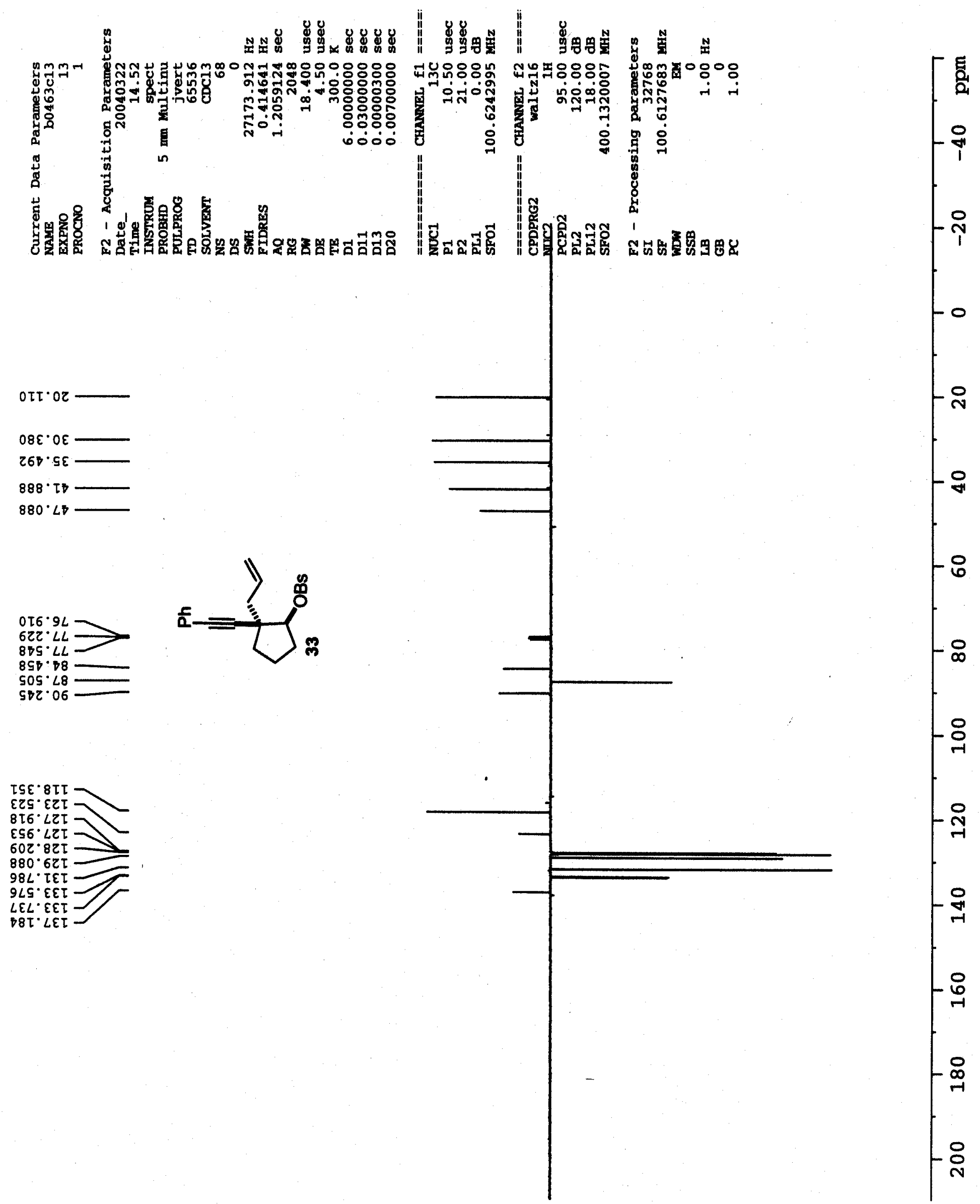

svz.06

D $8 \mathrm{~L}$ LEI 

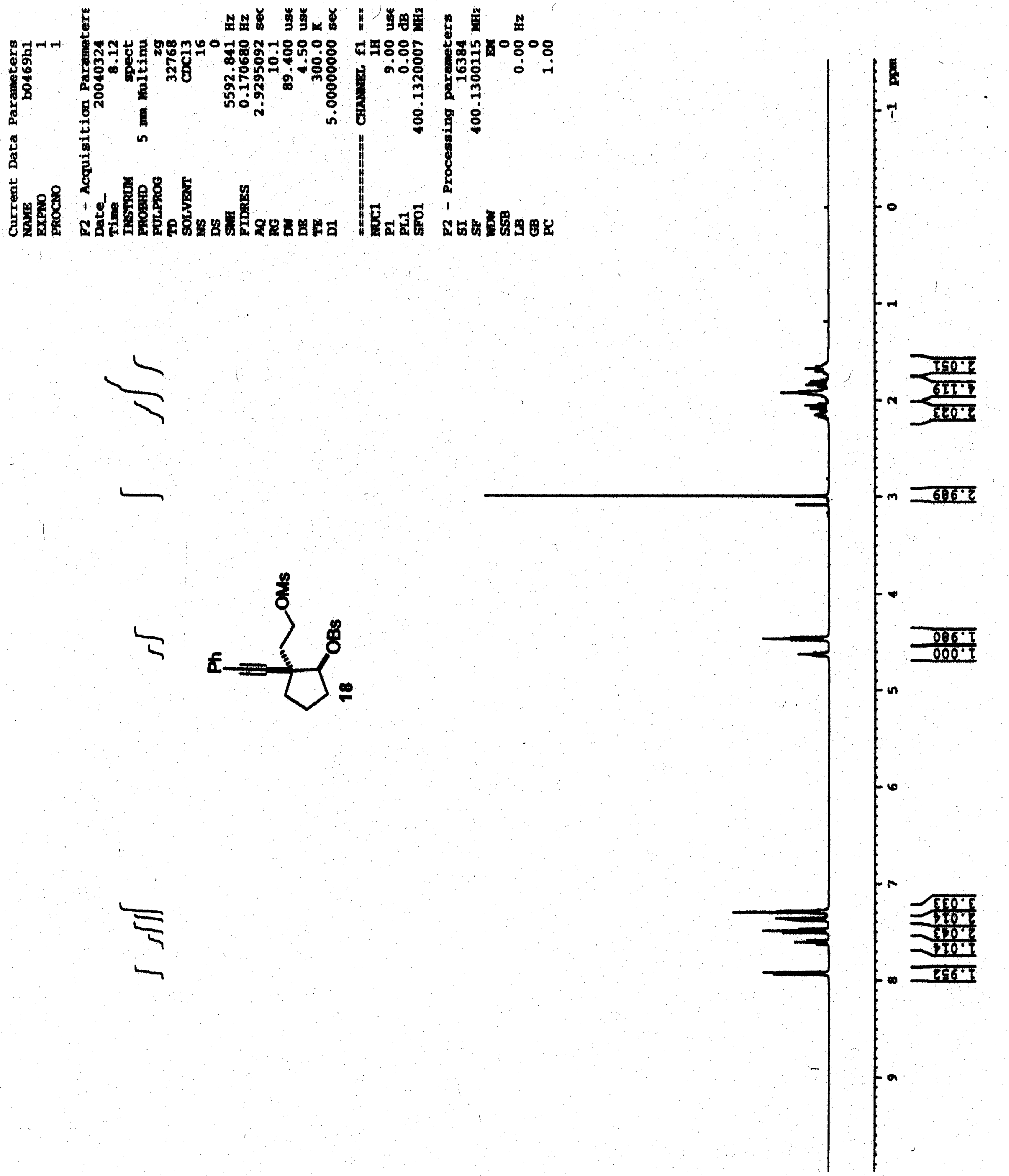

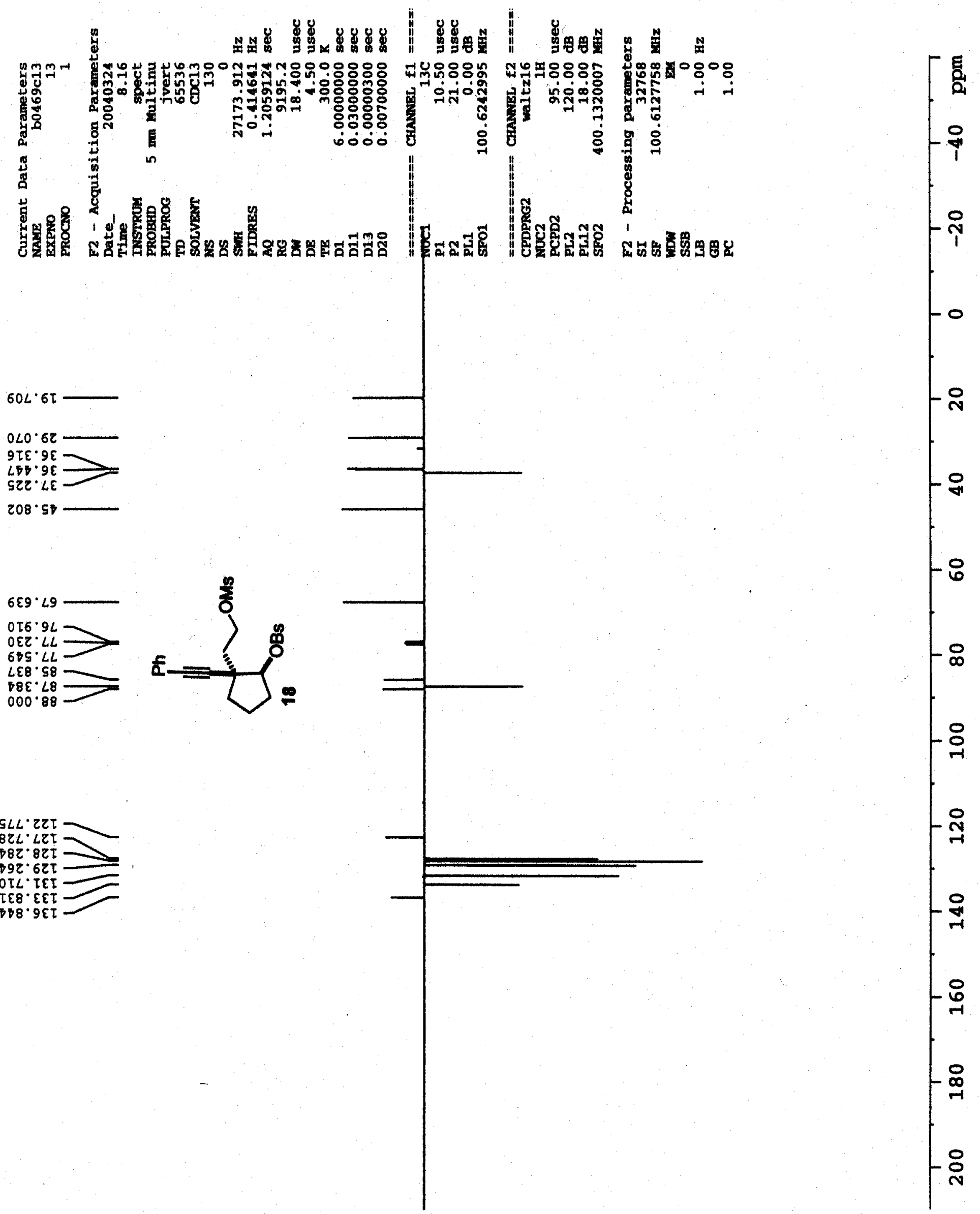

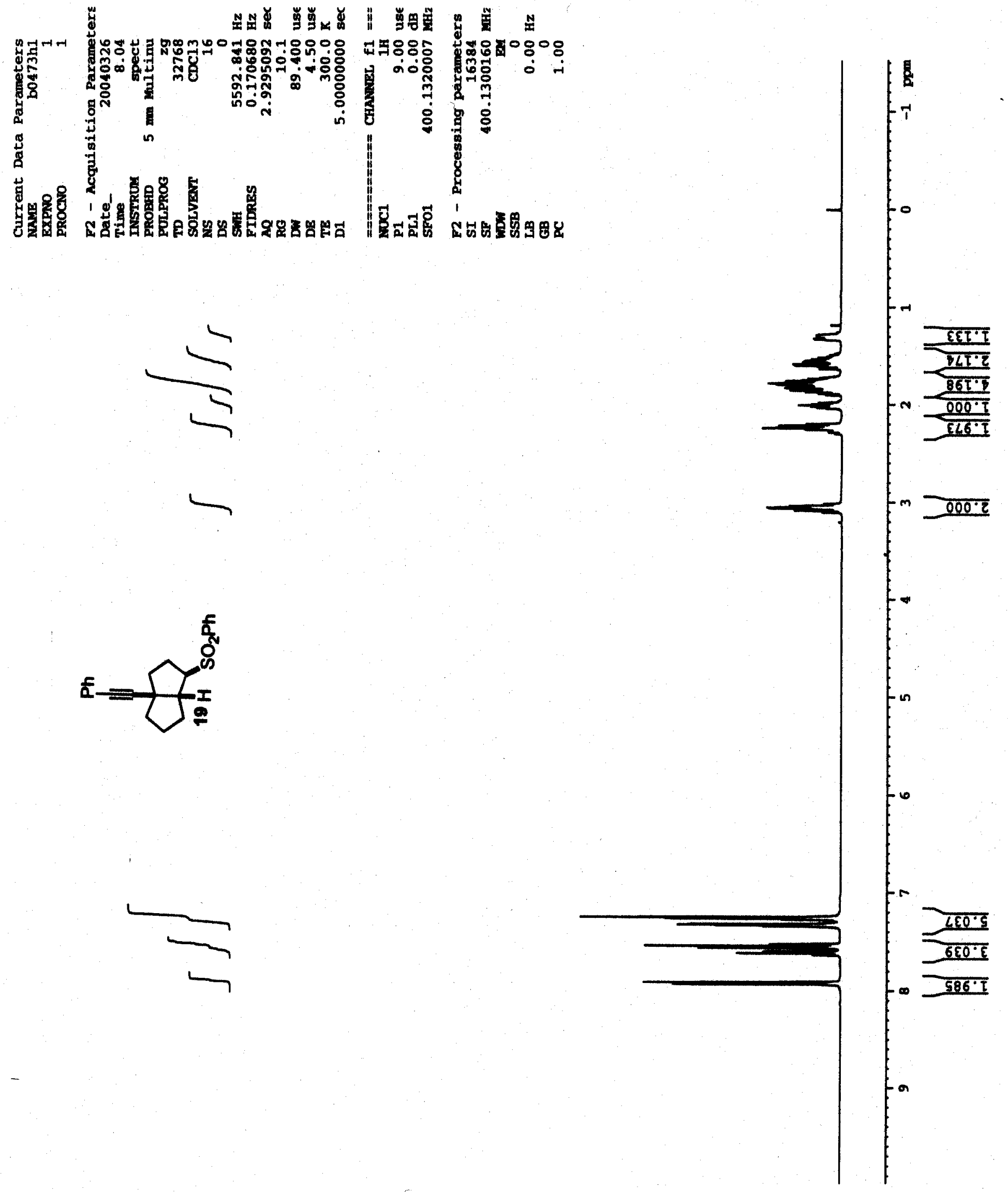

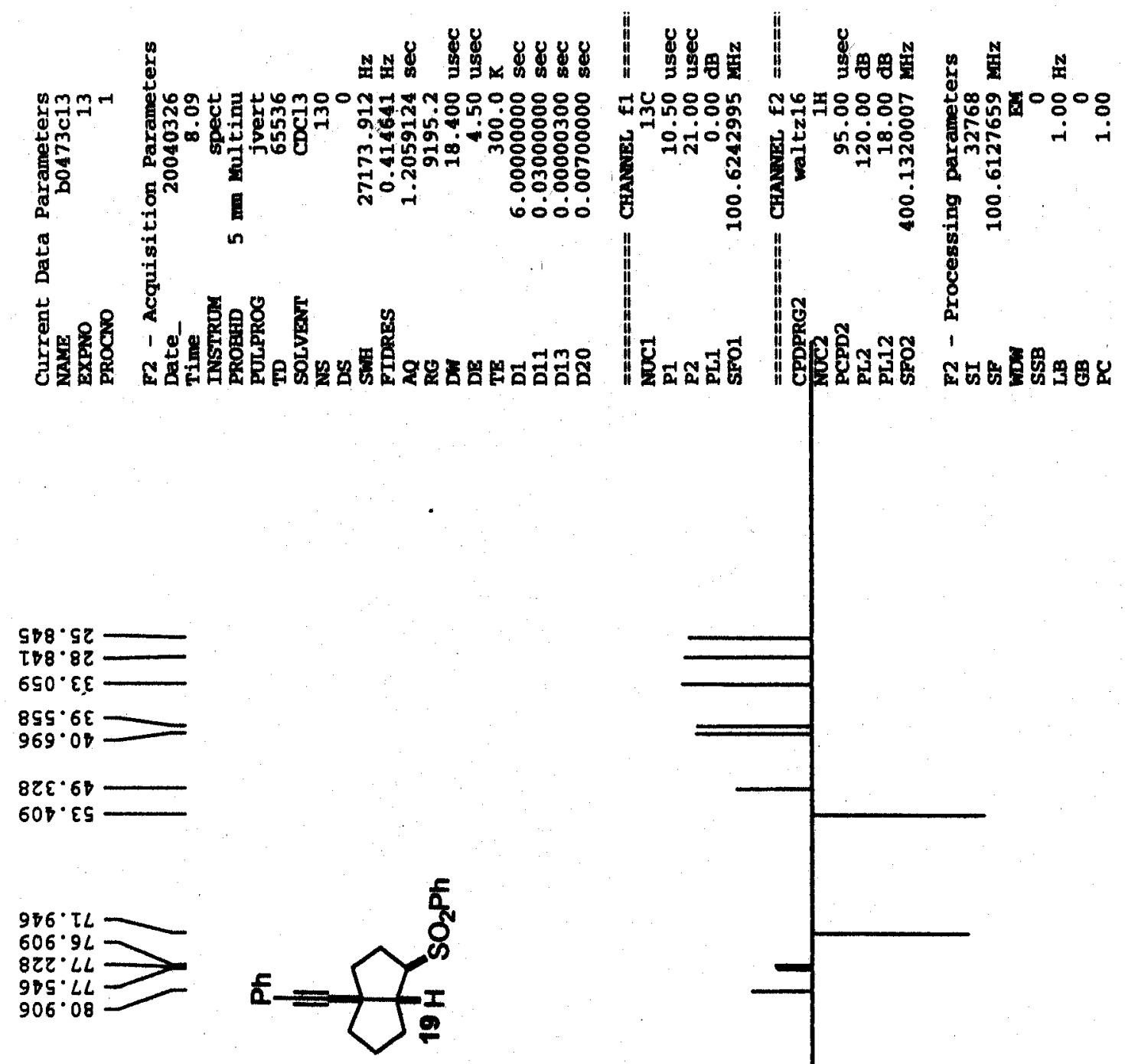

$695 \cdot 96$
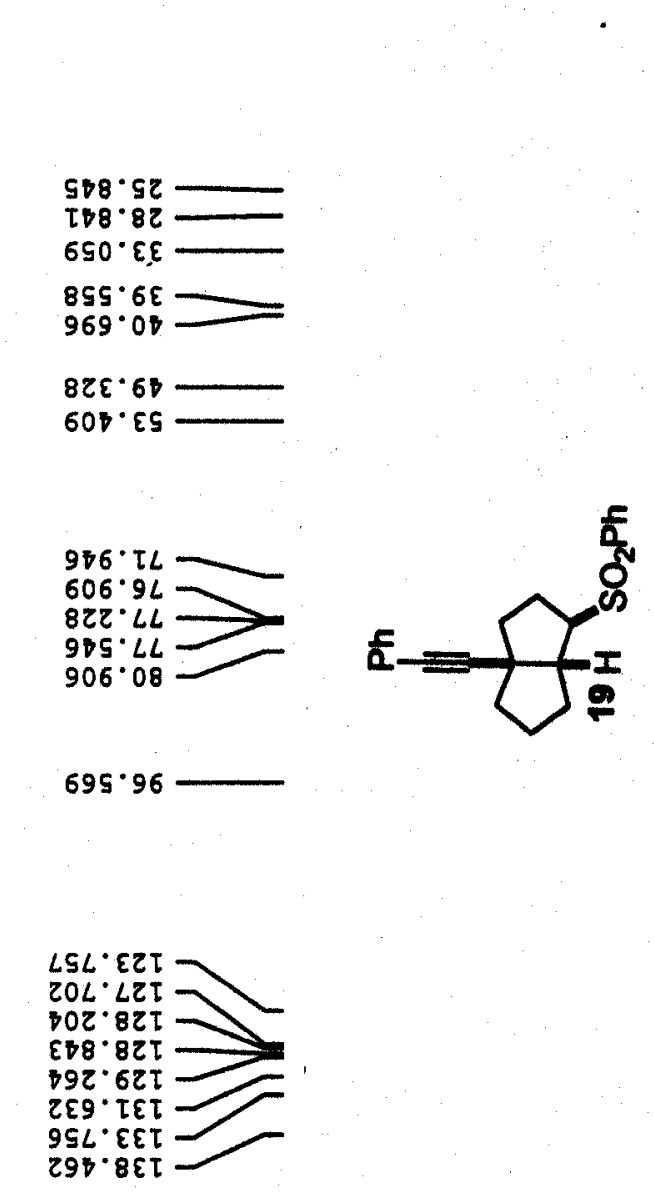